\title{
Price and Service Competition in a Dual-Channel Supply Chain with Product Customization
}

\author{
Jian Wang $\mathbb{D}^{1,2}$ and Huijuan Jiang ${ }^{1,2}$ \\ ${ }^{1}$ School of Artificial Intelligence and Automation, Huazhong University of Science and Technology, Wuhan 430074, China \\ ${ }^{2}$ Key Laboratory of Image Processing and Intelligent Control (Huazhong University of Science and Technology), \\ Ministry of Education, Wuhan 430074, China
}

Correspondence should be addressed to Jian Wang; wj0826_can@mail.hust.edu.cn

Received 8 September 2020; Revised 22 December 2020; Accepted 27 February 2021; Published 26 March 2021

Academic Editor: Pietro De Lellis

Copyright (C) 2021 Jian Wang and Huijuan Jiang. This is an open access article distributed under the Creative Commons Attribution License, which permits unrestricted use, distribution, and reproduction in any medium, provided the original work is properly cited.

\begin{abstract}
This paper considers a dual-channel supply chain with product customization. One manufacturer and one retailer are involved. The online direct sales channel sells standard and customized products, and the offline retail channel sells standard products. The prices and service levels of products sold via different channels are differentiated, and the customization level which influences the customization cost and choices of customers is decided by the manufacturer. Three game models are proposed: the manufacturer Stackelberg (MS) model, the retailer Stackelberg (RS) model, and the Nash game model. The price and service decisions of the players are derived. Meanwhile, a service-cost-sharing contract is designed for the MS model. The impacts of price and service competition, service cost, and customers sensitivity to the customization level on the optimal decisions are investigated. Through the numerical analysis, we find that, among the three models, the manufacturer Stackelberg model is the most beneficial game structure for the overall supply chain but has the largest revenue gap between the two members. Second, under price competition and service competition, the manufacturer should differentiate the prices and services for direct sales standard products and customized products according to his market status. Third, the manufacturer should increase customization expenditures to construct his customization production line and provide more diversified products when consumers are more sensitive to product customization.
\end{abstract}

\section{Introduction}

Owing to the popularity of the Internet, the e-commerce industry is gradually maturing, and e-commerce websites, such as Amazon and Taobao, enable customers to purchase most products, including electronic and digital products, food, clothing, and more, through the Internet. However, the prosperity of e-commerce platforms forces the traditional retail industry to confront unprecedented opportunities and challenges. Some newborn small manufacturers specialize in providing products only for online sales and occupy a greater market share, which has shaken the market position of traditional manufacturers (only selling products via brick-and-mortar retailers). In order to reduce the negative impacts brought by e-commerce, many manufacturers are exploring new sales channels in addition to maintaining traditional offline sales channels [1]. Some large companies, such as Estee Lauder, Xerox, and Hewlett-Packard, choose to build up their own shopping websites and sell their products directly to end users without a middleman [2]. The direct sales mode helps manufacturers avoid allocating income to a middleman and instead build their own market, making it faster and more accurate for manufacturers to capture market information and regulate strategies opportunely $[2,3]$. The structure of selling products through both an online direct sales channel and an offline retail channel is called a dual-channel supply chain (SC). The emerging dualchannel sales mode helps manufacturers maintain their advantages despite fierce market competition and form customer brand loyalty. 
The combination of online and offline sales fixes the problem of distance. Efficient logistics services enable customers to buy products across cities or even across countries using an online shopping platform [1]. Consumers can enjoy the same maintenance services while purchasing products at a lower price. The products sold via an online direct sales channel are the same as the products sold by a retailer in appearance and function but can be distinguished by their delivery cycle and logistics service, which makes returns or exchanges occur more frequently. In this case, it is difficult for manufacturers to determine which products should be sold online, whether products that are sold both online and offline should be differentially priced, and how to provide the same services for products sold online. These problems desperately need to be solved by manufacturers applying the dual-channel sales mode. Moreover, in an SC structure, all supply chain members are not isolated, but rather they are dependent on each other [4]. However, with the introduction of the online direct sales channel, horizontal competition between the manufacturer and the retailer emerges. In a dual-channel SC, considering differentiated prices and services provided for products sold via different channels, price competition and service competition exist between the online channel and retail channel. Some scholars pay attention to how these competitions change the operating decisions and profits of decision-makers. Once the diversity of products in the dual-channel SC is considered, the impacts of price competition and service competition on decisions and profits can be more complicated.

As economic prosperity has increased, people are no longer satisfied with pipelining products; consumers require product variety and flexibility. In response, some large manufacturers adopt a mixed production strategy, that is, adding the production of customized products while continuing to manufacture standard products. Standard products are produced to meet most consumers' demands while product customization provides customers with alternative varieties of products and makes them feel unique [5]. The process of manufacturing customized products is as follows. First, the customer clarifies the characteristics of their demand. Then the manufacturer responds to the demand and produces the customized product. Later, the customized product will be delivered to the customer. This kind of sales mode makes customized products more suitable for being sold via the online channel. Thanks to the development of the Internet and logistics, currently, some companies (such as Nike and Dell) provide customized products online [6]. The online sales of customized products eliminate the need for consumers to go back and forth, providing great convenience; however, customers may have to pay a higher price to obtain these unique, customized products. These issues plague decision-makers on how to choose the sales channel, price the customized products, and provide services.

However, despite the more diversified sales channels and products, market competition among different products and among different sales channels has resulted in many disadvantages, which can harm the profits of supply chain members. The two main competitive issues in the supply chain are about prices and services. The services provided by retailers and manufacturers have significant influences on customers' demand, channel selection, and channel loyalty. Most published literature investigates the price competition for a single kind of product between two sales channels. Fierce price competition motivates decision-makers to lower the price to increase the sales volume. Similarly, service competition urges service providers to improve the service levels of their products, which results in higher costs. It is worth investigating the optimal price and service decisions so that SC members can avoid excessive costs while maintaining their sales volumes. In a dual-channel SC with product customization, the selling prices and service provided by the manufacturer and the retailer should be differentiated considering the product variety and sales channel diversity. For the offline channel, retailers can provide some value-added services to attract repeat consumers. For example, Huawei provides free screen protectors for consumers who purchase cellphones in physical stores. For the online channel, manufacturers can provide some special services for consumers who purchase customized products. They can track the needs of consumers continuously and allow consumers to intervene in the production process of products as soon as possible so that consumers can obtain products tailored to their individual needs. Investigating price competition and service competition can help supply chain members make better decisions to maintain market share and earn more profits. Moreover, an appropriate coordination mechanism should be designed to reduce the negative impacts brought by the competition and coordinate a dual-channel SC [7]. For a dual-channel SC, a revenuesharing contract is one of the most common contracts to improve SC performance [8].

A dual-channel SC including one manufacturer and one retailer is considered. The manufacturer produces both standard products and customized products. The customized products are sold by the manufacturer online directly. In addition, the standard products are sold both via the retailer and directly. For example, Dell enables customers to order customized computers through its official websites while selling standard edition computers through both online and offline channels [9]. Three models of this dualchannel supply chain are proposed: (1) the manufacturer Stackelberg (MS) model, (2) the retailer Stackelberg (RS) model, and (3) the Nash game model. We investigate how price competition and service competition influence SC members' price and service decisions in a dual-channel SC selling both standard products and customized products. We find that, under price competition and service competition, the manufacturer should differentiate the prices and services for direct sales of standard products and customized products according to his market status. In addition, we find that the manufacturer should increase customization expenditures to construct his customization production line and provide more diversified products when consumers are more sensitive to product customization. In addition, the impacts of the service cost coefficient on price and service decisions are investigated. Numerical studies imply that the high service cost coefficient of the manufacturer results in 
more profits for the overall SC in the RS model and the Nash game model. By comparing the three models, we find that the MS model performs the best regarding total profits but results in the largest revenue gap between the two members.

The contributions of this paper are as follows. First, product customization is integrated into a dual-channel SC. Standard products are sold both online and offline, and customized products are sold online. The results show that it is essential to sell customized products to maintain the income of the manufacturer. Furthermore, the customization level is defined to measure the customization capability provided by the manufacturer, which also affects the customers' preferences for customized products and the customization costs. Second, three kinds of game models are established to simulate the different power structure situations in the real market environment. Then, a service-costsharing contract is provided for the MS model to coordinate the supply chain under price and service competition. Third, the impacts of price and service competition on supply chain members' decisions are analyzed to maximize all parties' revenues. The competition considered in this paper exists not only between two products, but also between the online and offline channels. The distinct competition between different products and between different sales channels is vital for SC members to recognize the impacts of price and service competition and help all parties cooperate greatly.

The remaining structure is as follows. Section 2 surveys the related literature. The notations and assumptions are defined in Section 3. In Section 4, three different models of a dual-channel SC are formulated along with equilibrium decisions. Section 5 presents the service-cost-sharing contract. Numerical analysis and managerial insights are derived in Section 6. Section 7 draws the conclusions.

\section{Literature Review}

This study relates to the literature following: one is about the price and service competition or coordination in an SC, one relates to decisions in a dual-channel SC, and the third one is about the product customization.

The equilibrium decision issue has become a significant problem in SC management when price and service competition exists [10]. As two of the hottest topics in SC management, both pricing decisions and service decisions in an SC have been investigated by many scholars. Most of the time, price and service decision problems are investigated in a traditional SC with one manufacturer and one retailer. Sun et al. [10] propose an SC model with the manufacturer providing a compulsory basic quality assurance service for free and the retailer providing a paid optional quality assurance service. They derive the equilibrium decisions under the two cases where the manufacturer or the retailer determines the optional after-sales service level. By introducing a third-party cold-chain logistics service provider, $\mathrm{Yu}$ and Xiao [11] investigate the price and service decisions of a fresh agri-products SC. Affected by the quantity loss and quality loss of agri-products, they examine the impacts of the channel power structure on the equilibrium prices and service levels.
Extreme price and service competition always exists in an SC with multiple manufacturers or multiple retailers. Lu et al. [12] consider an SC with two manufacturers producing different but substitutable products and selling them to the same retailer. They investigate three bargain power structures and find that the vertical Nash scenario provides the highest service level for consumers, which is different from this paper's outcomes. Furthermore, the competitor's profits increase as the market base of one product increases, but the increment is smaller. Some researchers, such as $\mathrm{Wu}$ [13] and Wang and Wang [14], pay attention to price and service competition in a closed-loop supply chain (CLSC). They both consider a CLSC composed of a manufacturer, a remanufacturer, and a retailer. The new products and remanufactured products compete on sales prices and service levels, which are determined by the manufacturer and the remanufacturer. Wu [13] concludes that price competition is beneficial for the remanufacturer and the retailer, while service competition is profitable for the retailer. Customer acceptance of remanufactured products is introduced in the study of Wang and Wang [14]. They derive the optimal prices and service levels in the manufacturer Stackelberg scenario, retailer Stackelberg scenario, and Nash equilibrium scenario. The price and service competition between two retailers always relates to the sales channels or the types of products. Dan et al. [15] consider two retailers in the dual-channel SC where one retailer is a strong brick-and-mortar retailer and the other retailer is an electronic retailer. Under price and service competition, the influence of retailer power is investigated and some competitive strategies for the retailer within an e-commerce environment are proposed. In the paper of Han et al. [16], two competing retailers sell two substitutable products produced by one manufacturer and compete on price and service, while the manufacturer provides service. Indeed, price and service competition exists not only in the SC, but also between two supply chains. Competition between supply chains may lead to a lower price to acquire a greater market demand or to hold higher inventory.

In recent years, as e-commerce has gradually become popular, a special SC structure, called a dual-channel SC, has been studied frequently. Similarly, price and service competition and coordination problems in a dual-channel SC have been studied by some scholars, and the most popular method to coordinate an SC is to draw a coordination contract [17]. Ma et al. [18] explore the optimal decisions of two manufacturers who compete on price and local advertising investments and propose a cost-sharing contract. In the study of Li et al. [19], a dual-channel SC with green products is considered. They compare the equilibrium outcomes of the single channel and dualchannel, investigate the channel competition issue in the decentralized dual-channel SC, and draw a contrary result of "double marginalization". Under the disruptions of demand and costs on pricing, Tang et al. [20] investigate the channel competition and coordination in a dual-channel SC. It is found that moderate variations and boosts in the revenue-sharing contract can coordinate a disrupted dual- 
channel SC. Some scholars only consider the service competition problem in a dual-channel SC. As a valueadded kind of after-sales service, warranty services have been studied frequently recently. In the paper of Dan et al. [21], the manufacturer provides a warranty service while the retailer offers free value-added service. Under the effect of both warranty and free services, they examine the optimal warranty service level and the value-added service competition between members. The result shows that service competition decreases as the warranty service level increases. Apart from considering price competition or service competition separately, some researchers also consider price and service competition simultaneously $[15,22]$. A dual-channel SC model with return policies is proposed by Ren et al. [23]. The price and service competition between channels is considered, and a new contract is designed, forming a win-win situation for the SC members. Sarmah et al. [24] study the retail price and warranty policy competition and coordination problems under a duopoly situation between two boundedly rational agents. They find that competition occasionally results in greater profits than coordination in terms of "social welfare". Xie et al. [1] investigate the revenue-cost-sharing contract in a dual-channel CLSC under the Stackelberg game, and they find that the sharing channel cost encourages the retailer to be involved in service and recovery.

The literature studies that study product customization are quite limited. Usually, the dual-channel sales model with product customization assumes that standard products are sold via the retailer while customized products are sold online [25, 26]. Under this condition, Xiao et al. [26] propose a dual-channel SC model to analyze the impacts of product variety and channel structure on the optimal decisions. $\mathrm{Li}$ et al. [25] examine whether the manufacturer should provide customized products online. They focus on channel competition and vertical competition to derive pricing decisions. Batarfi et al. [27] compare the price and inventory decisions in a single channel or dual channel, both with product customization, and confirm that the dual-channel sales mode is better for an SC with product customization. Later, the product customization issue is investigated in a dualchannel CLSC [28]. In the customized dual-channel SC model of Li [29], the ecology industry is supposed to be the major member. They find that when product customization is considered, the price sensitivity of direct channel demands and the price sensitivity between channels affect profit.

The literature studies reviewed above all contribute to their respective fields and are worth studying; however, they only focus on price and service competition, a dual-channel SC, and product customization separately. Our paper is among the first pieces of research that considers price and service competition and coordination in a dual-channel SC with product customization. By introducing the customization level and the customization cost coefficient, the impacts of the degree of customization on price and service decisions are analyzed. Furthermore, by analyzing the effects of price and service competition intensity and by comparing the prices and service levels of two products, managerial insights are offered.

\section{Model Basics}

3.1. Problem Formulation. In this section, the basic model settings are given. A traditional dual-channel SC containing a manufacturer and a retailer is considered, as in Figure 1. The manufacturer produces standard products and customized products, both of which are substitutable in their basic functions. The customization level $\mu$ that measures the customization capability of the manufacturer is decided by the manufacturer, and this level also affects the customization costs of the manufacturer and other decisions. In addition, the customized products include some specific custom features required by the consumers to meet the various demands of individual consumers. The manufacturer sells the standard products directly via an online channel at $p_{m}$ and wholesales the standard products to the retailer at $w$. Then, the retailer sells the standard products to the end users at $p_{r}$. The customized products are sold to consumers online at $p_{z}$. The dual-channel sales mode can satisfy consumers' demand for timeliness and geographic position. Moreover, the manufacturer provides service for the customized products at the service level $s_{z}$ and the standard products online at the service level $s_{m}$ while the retailer provides service for the standard products offline at the service level $s_{r}$. The service considered in this paper is assumed to include pre-sales and after-sales service. The manufacturer should provide a more sophisticated service for consumers who purchase customized products. Both prices and service levels for different products via different channels are differentiated to ensure profits since consumers' purchasing behavior is affected by selling prices, service levels, and the customization level. Therefore, price and service competition exists between the online and offline channels and between the two kinds of products. Based on the SC structure described above, three decentralized dualchannel supply chain models are proposed: the manufacturer Stackelberg model, the retailer Stackelberg model, and the Nash game model.

\subsection{Notations. Parameters used in this study are as follows:}

$c_{s}$ : unit production cost for standard products

$c_{z}$ : unit production cost for customized products

$D_{m}$ : consumer demand of standard products from the direct sales channel

$D_{r}$ : consumer demand of standard products from the retail channel

$D_{z}$ : consumer demand of customized products

$\alpha_{m}$ : market base for standard products from the direct sales channel

$\alpha_{r}$ : market base for standard products from the retail channel

$\alpha_{z}$ : market base for customized products

$\delta_{s}$ : price elasticity of standard products

$\delta_{z}$ : price elasticity of customized products

$\beta_{s}$ : service elasticity of standard products 


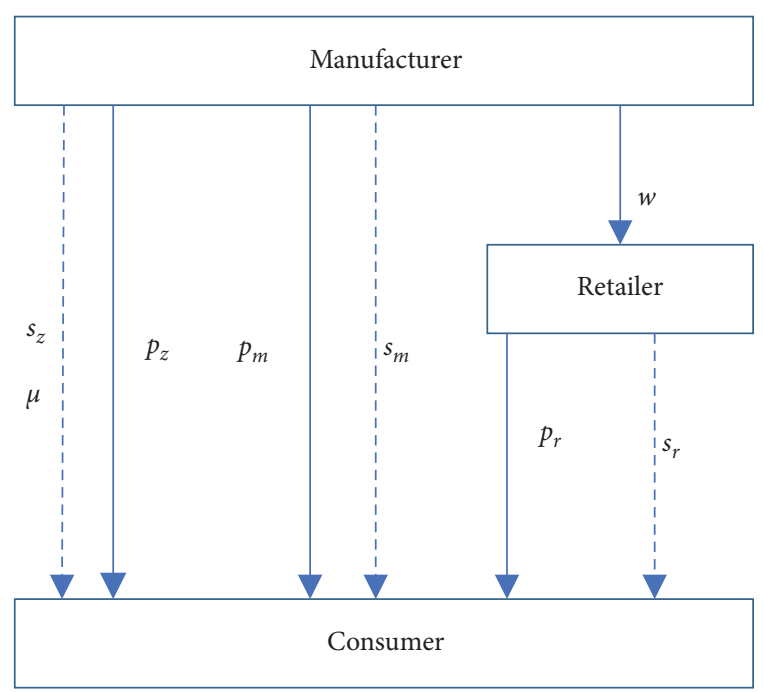

FIgUre 1: A dual-channel SC structure.

$\beta_{z}$ : service elasticity of customized products

$\gamma_{c}$ : price competition coefficient between two channels

$\gamma_{p}$ : price competition coefficient between standard products and customized products

$\theta_{c}:$ service competition coefficient between two channels

$\theta_{p}$ : service competition coefficient between standard products and customized products

$\varphi_{0}$ : service-cost-sharing ratio

$\varphi_{1}$ : customer sensitivity coefficient to the level of customization

$\varphi_{2}:$ customer cross coefficient to the level of customization

$C$ : customization scaling parameter provided by the manufacturer

$\eta_{1}$ : service cost coefficient of the manufacturer

$\eta_{2}$ : service cost coefficient of the retailer

$\Pi_{m}$ : the profit function for the manufacturer

$\Pi_{r}$ : the profit function for the retailer

$\Pi$ : the profit function for the overall supply chain

Decision variables used in this study are as follows:

$w$ : wholesale price of standard products

$p_{r}$ : retail price of standard products in the retail channel

$p_{m}$ : direct sales price of standard products in the direct sales channel

$p_{z}$ : direct sales price of customized products

$\mu$ : level of customization provided by the manufacturer

$s_{r}$ : service level of standard products in the retail channel

$s_{m}$ : service level of standard products in the direct sales channel

$s_{z}$ : service level of customized products $s$ : service level of all products; this variable only exists in the coordination model when the retailer provides the same service for both standard products via dual channels and customized products

3.3. Assumptions. Before formulating the price and service model, the basic assumptions are proposed to simplify this issue.

Assumption 1. The demand function is assumed to be linear and depends on both selling prices and service levels, which is common in the literature studies about supply chain price and service competition issues [16, 30,31]. The effects of both price competition and service competition between online and offline channels as well as between standard products and customized products are considered. The direct sales demand for standard products is $D_{m}=\alpha_{m}-\delta_{s} p_{m}+$ $\gamma_{c}\left(p_{r}-p_{m}\right)+\gamma_{p}\left(p_{z}-p_{m}\right)+\beta_{s} s_{m}-\theta_{c}\left(s_{r}-s_{m}\right)-\theta_{p}\left(s_{z}-s_{m}\right)$ $-\varphi_{2} \mu$, the retail demand for standard products is $D_{r}=\alpha_{r}-$ $\delta_{s} p_{r}+\gamma_{c}\left(p_{m}-p_{r}\right)+\left(\gamma_{c}+\gamma_{p}\right)\left(p_{z}-p_{r}\right)+\beta_{s} s_{r}-\theta_{c}\left(s_{m}-s_{r}\right)-$ $\left(\theta_{c}+\theta_{p}\right)\left(s_{z}-s_{r}\right)-\varphi_{2} \mu$, and the demand for customized products is $D_{z}=\alpha_{z}-\delta_{z} p_{z}+\gamma_{p}\left(p_{m}-p_{z}\right)+\left(\gamma_{c}+\gamma_{p}\right)\left(p_{r}-\right.$ $\left.p_{z}\right)+\beta_{z} s_{z}-\theta_{p}\left(s_{m}-s_{z}\right)-\left(\theta_{c}+\theta_{p}\right)\left(s_{r}-s_{z}\right)+\varphi_{1} \mu$.

Assumption 2. The cost function for service is $(1 / 2) \eta_{i} s_{j}^{2}$, where subscript $i$ can be 1 or 2 , denoting the manufacturer or the retailer, respectively. Subscript $j$ can be $r, m$, or $z$, which denotes standard products sold offline, standard products sold online, or customized products, respectively. The quadratic form represents diminishing returns on service expenditures and is widely applied in papers about service cost $[16,32,33]$. Similarly, the customization cost function is $(1 / 2) C \mu^{2}$.

Assumption 3. The players' pricing decisions are assumed to be made after the service level decisions. This sequence of events is commonly used and accepted in the literature studies about price-service decisions in the supply chain $[13,33,34]$.

Assumption 4. The manufacturer and the retailer are assumed to decide service levels sequentially, which is proved to be better than simultaneously deciding service levels [34]. What is more, the manufacturer's optimal decision in service level is recommended to be made before the retailer's optimal service decision.

Assumption 5. All the SC members are risk-neutral and profit-maximizing.

Assumption 6. Both the manufacturer and the retailer are exposed to the same information.

\section{Three Decentralized Models}

In this section, three different SC structures are modeled to investigate the impacts of price and service competition. 
Given the market demand functions, the profit functions are obtained as follows:

$$
\begin{aligned}
\Pi_{m}\left(w, p_{m}, p_{z}, s_{m}, s_{z}, \mu\right)= & \left(w-c_{s}\right) D_{r}+\left(p_{m}-c_{s}\right) D_{m} \\
& +\left(p_{z}-c_{z}\right) D_{z} \\
& -\frac{1}{2} \eta_{1} s_{m}^{2}-\frac{1}{2} \eta_{1} s_{z}^{2}-\frac{1}{2} C \mu^{2}, \\
\Pi_{r}\left(p_{r}, s_{r}\right)= & \left(p_{r}-w\right) D_{r}-\frac{1}{2} \eta_{2} s_{r}^{2} .
\end{aligned}
$$

In equation (1), the profit of the manufacturer consists of the revenue for selling two kinds of products online as well as wholesaling standard products to the retailer and deducting the service cost for two kinds of products sold online as well as the customization cost. In equation (2), the retailer's profit is equal to the profit earned by selling standard products minus the service cost for the standard products via the retail channel.
4.1. Manufacturer Stackelberg Model. In the MS model, the manufacturer is the Stackelberg leader and the retailer is assumed to be the follower. This type of power structure exists when the manufacturer has greater bargaining power, such as Apple. The sequence of decision-making is shown in Figure 2(a). First, the manufacturer determines the service level of the standard products sold via the online channel $s_{m}$, the service level of the customized products $s_{z}$, and the customization level $\mu$ to maximize his revenues. Then, the retailer sets the optimal service level for the standard products via the retail channel $s_{r}$. In the next stage, the manufacturer determines the optimal wholesale price of the standard products $w$, the optimal direct sales price of the standard products $p_{m}$, and the optimal price of the customized products $p_{z}$. After observing the manufacturer's decision, the retailer sets the optimal retail price of the standard products sold offline $p_{r}$ to maximize her profits.

Backward deduction is applied to derive the equilibrium solutions. In the last stage, since $\left(\partial^{2} \Pi_{r} / \partial p_{r}^{2}\right)=-2\left(\delta_{s}+2 \gamma_{c}+\right.$ $\left.\gamma_{p}\right)<0, \Pi_{r}$ is concave in $p_{r}$. Differentiate $\Pi_{r}$ with respect to $p_{r}$ in equation (2) to get the optimal $p_{r}$.

$$
p_{r}^{*}\left(w, p_{m}, p_{z}\right)=\frac{\alpha_{r}+\gamma_{c} p_{m}+\left(\gamma_{c}+\gamma_{p}\right) p_{z}+\left(A_{3}+\theta_{c}\right) s_{r}-\theta_{c} s_{m}-\left(\theta_{c}+\theta_{p}\right) s_{z}-\varphi_{2} \mu}{2\left(A_{1}+\gamma_{c}\right)}+\frac{w}{2}
$$

The definition of new notations $A_{1}$ and $A_{3}$ is given in the Appendix, Proof of Proposition 1.
Proposition 1. $\Pi_{m}$ is jointly concave in $w, p_{m}$, and $p_{z}$ under the given service levels provided that

$$
\begin{aligned}
\gamma_{c} \gamma_{p}< & \frac{\left[2\left(\delta_{s}+2 \gamma_{c}+\gamma_{p}\right)\left(\delta_{s}+\gamma_{c}+\gamma_{p}\right)-\gamma_{c}^{2}\right]\left[2\left(\delta_{z}+\gamma_{c}+2 \gamma_{p}\right)-\left(\gamma_{c}+\gamma_{p}\right)^{2} / \delta_{s}+2 \gamma_{c}+\gamma_{p}\right]}{4\left(\gamma_{c}+\gamma_{p}\right)} \\
& -\frac{\left(\delta_{s}+\gamma_{c}+\gamma_{p}\right)\left(\gamma_{c}+\gamma_{p}\right)}{2}-\frac{\left(\delta_{z}+\gamma_{c}+2 \gamma_{p}\right) \gamma_{c}^{2}}{2\left(\gamma_{c}+\gamma_{p}\right)}-\frac{\left(\delta_{s}+2 \gamma_{c}+\gamma_{p}\right)\left[2 \gamma_{p}+\gamma_{c}\left(\gamma_{c}+\gamma_{p}\right) / \delta_{s}+2 \gamma_{c}+\gamma_{p}\right]^{2}}{4\left(\gamma_{c}+\gamma_{p}\right)} .
\end{aligned}
$$

Given the service levels and the customization level, Proposition 1 guarantees the existence of the manufacturer's optimal price decisions. The unique optimal solutions are obtained by

$$
\begin{aligned}
& w^{*}=\frac{B_{1}+D_{1} s_{r}+E_{1} s_{m}+F_{1} s_{z}+G_{1} \mu}{A_{6}}, \\
& p_{m}^{*}=\frac{B_{2}+D_{2} s_{r}+E_{2} s_{m}+F_{2} s_{z}+G_{2} \mu}{A_{6}}, \\
& p_{z}^{*}=\frac{B_{3}+D_{3} s_{r}+E_{3} s_{m}+F_{3} s_{z}+G_{3} \mu}{A_{6}}, \\
& p_{r}^{*}=\frac{B_{4}+D_{4} s_{r}+E_{4} s_{m}+F_{4} s_{z}+G_{4} \mu}{A_{6}} .
\end{aligned}
$$

The definition of new notations mentioned above such as $A_{6}, B_{1}, D_{1}, E_{1}, F_{1}$, and $G_{1}$ is given in the Appendix, Proof of Proposition 1.

Next, we substitute the optimal prices $w^{*}, p_{m}^{*}, p_{z}^{*}$, and $p_{r}^{*}$ into equation (1) and equation (2) and then differentiate $\Pi_{r}$ with respect to $s_{r}$.

Proposition 2. $\Pi_{r}$ is concave in $s_{r}$ provided that

$$
\eta_{2}>\frac{2\left(D_{4}-D_{1}\right)}{A_{6}}\left[A_{3}+\theta_{c}+\frac{\gamma_{c} D_{2}+\left(\gamma_{c}+\gamma_{p}\right) D_{3}-\left(A_{1}+\gamma_{c}\right) D_{4}}{A_{6}}\right] \text {. }
$$

The unique optimal service level is derived as follows: 


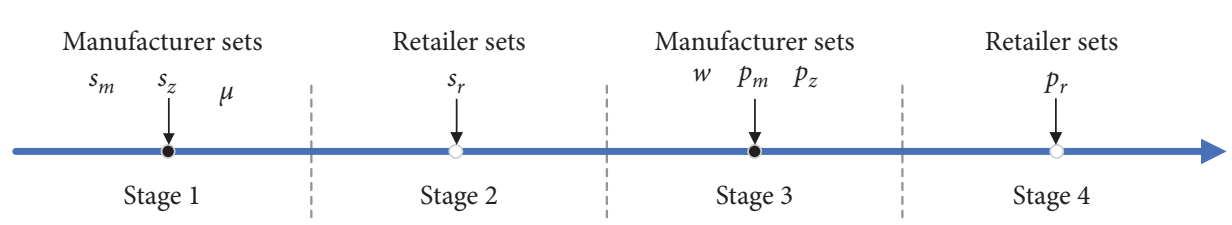

(a)

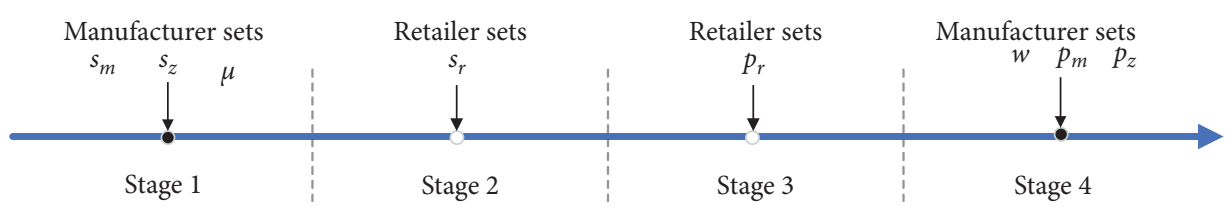

(b)

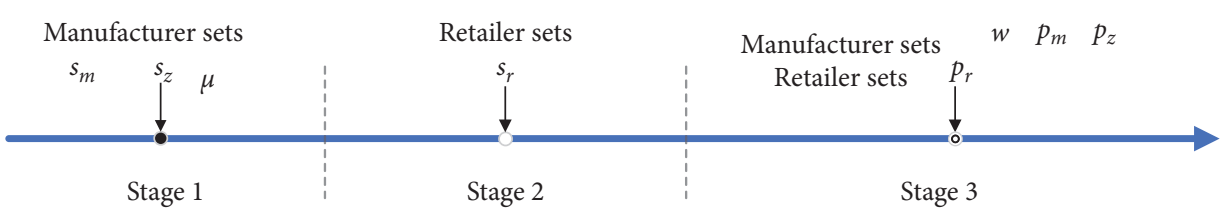

(c)

Figure 2: (a) Decision-making sequence in the MS model. (b) Decision-making sequence in the RS model. (c) Decision-making sequence in the Nash model.

$$
s_{r}^{*}=\frac{H_{1}+H_{2} s_{m}+H_{3} s_{z}+H_{4} \mu}{H_{5}} .
$$

The definition of new notations $\mathrm{H}_{1}, \mathrm{H}_{2}, \mathrm{H}_{3}, \mathrm{H}_{4}$, and $\mathrm{H}_{5}$ is given in the Appendix, Proof of Proposition 2.

Additionally, we substitute $s_{r}^{*}$ into $\Pi_{m}$ and differentiate $\Pi_{m}$ with respect to $s_{m}, s_{z}$, and $\mu$.
Proposition 3. $\Pi_{m}$ is jointly concave in $s_{m}$, $s_{z}$, and $\mu$ provided that $I_{2}<0, \quad I_{2} J_{3}-I_{3} J_{2}>0$ as well as $I_{2}\left(J_{3} K_{4}-\right.$ $\left.J_{4} K_{3}\right)+J_{2}\left(I_{4} K_{3}-I_{3} K_{4}\right)+K_{2}\left(I_{3} J_{4}-I_{4} J_{3}\right)<0$. Finally, the optimal $s_{m}, s_{z}$, and $\mu$ are derived as follows to maximize $\Pi_{m}$ :

$$
\begin{gathered}
s_{m}^{*}=\frac{I_{1}\left(J_{4} K_{3}-J_{3} K_{4}\right)+J_{1}\left(I_{3} K_{4}-I_{4} K_{3}\right)+K_{1}\left(I_{4} J_{3}-I_{3} J_{4}\right)}{I_{2}\left(J_{3} K_{4}-J_{4} K_{3}\right)+J_{2}\left(I_{4} K_{3}-I_{3} K_{4}\right)+K_{2}\left(I_{3} J_{4}-I_{4} J_{3}\right)}, \\
s_{z}^{*}=\frac{I_{1}\left(J_{2} K_{4}-J_{4} K_{2}\right)+J_{1}\left(I_{4} K_{2}-I_{2} K_{4}\right)+K_{1}\left(I_{2} J_{4}-I_{4} J_{2}\right)}{I_{2}\left(J_{3} K_{4}-J_{4} K_{3}\right)+J_{2}\left(I_{4} K_{3}-I_{3} K_{4}\right)+K_{2}\left(I_{3} J_{4}-I_{4} J_{3}\right)}, \\
\mu^{*}=\frac{I_{1}\left(J_{3} K_{2}-J_{2} K_{3}\right)+J_{1}\left(I_{2} K_{3}-I_{3} K_{2}\right)+K_{1}\left(I_{3} J_{2}-I_{2} J_{3}\right)}{I_{2}\left(J_{3} K_{4}-J_{4} K_{3}\right)+J_{2}\left(I_{4} K_{3}-I_{3} K_{4}\right)+K_{2}\left(I_{3} J_{4}-I_{4} J_{3}\right)} .
\end{gathered}
$$

The definition of new notations mentioned above, such as $I_{1}$, $J_{1}$, and $K_{1}$, is given in the Appendix, Proof of Proposition 3.

4.2. Retailer Stackelberg Model. In the RS model, the retailer leads the Stackelberg game, and the manufacturer is the follower. The RS scenario occurs when the retailer has more bargaining power than the manufacturer. The retailer can be a company such as Amazon, and the manufacturers can be some small companies. The decision-making sequence is as shown in Figure 2(b). The manufacturer first determines the service level of the standard products sold online $s_{m}$, the service level of the customized products $s_{z}$, and the customization level $\mu$, and then the retailer sets the optimal service level for the standard products sold offline $s_{r}$. In the next stage, the retailer sets the optimal retail price of the standard products sold offline $p_{r}$. Then, the manufacturer sets the optimal wholesale price of the standard products $w$, the optimal direct sales price of the standard products $p_{m}$, and the optimal price of the customized products $p_{z}$ to maximize his profits. In this model, the manufacturer makes his best reaction to the former decisions. The manufacturer's profits increase as the wholesale price of the standard products increases, and the wholesale price should be lower than the retail price of the standard products. Therefore, in both the retailer Stackelberg model and the Nash game model, $w$ is assumed to be equal to $\left(\left(p_{r}+c_{s}\right) / 2\right)$, which is applied in the studies of Xie and Neyret [35] and Giri et al. [2]. We use backward deduction to solve the problem.

Proposition 4. $\Pi_{m}$ is jointly concave in $p_{m}$ and $p_{z}$ under the given service levels and customization level. The optimal $p_{m}$ and $p_{z}$ are derived as follows: 


$$
\begin{aligned}
p_{m}^{*}= & \frac{2 A_{2} \alpha_{m}+2 \gamma_{p} \alpha_{z}-\gamma_{p} c_{s}\left(\gamma_{c}+3 \gamma_{p}\right)+2 A_{2} c_{s}\left(A_{1}-(1 / 2) \gamma_{c}\right)+3\left[\gamma_{p}\left(\gamma_{c}+\gamma_{p}\right)+A_{2} \gamma_{c}\right] p_{r}}{4\left(A_{1} A_{2}-\gamma_{p}^{2}\right)} \\
& +\frac{\left(A_{2} A_{3}-\gamma_{p} \theta_{p}\right) s_{m}-\left[\gamma_{p}\left(\theta_{c}+\theta_{p}\right)+A_{2} \theta_{c}\right] s_{r}+\left(\gamma_{p} A_{4}-A_{2} \theta_{p}\right) s_{z}+\left(\gamma_{p} \varphi_{1}-A_{2} \varphi_{2}\right) \mu}{2\left(A_{1} A_{2}-\gamma_{p}^{2}\right)}, \\
p_{z}^{*}= & \frac{2 \gamma_{p} \alpha_{m}+2 A_{1} \alpha_{z}-A_{1} c_{s}\left(\gamma_{c}+\gamma_{p}\right)-\gamma_{c} \gamma_{p} c_{s}+3\left[A_{1}\left(\gamma_{c}+\gamma_{p}\right)+\gamma_{c} \gamma_{p}\right] p_{r}}{4\left(A_{1} A_{2}-\gamma_{p}^{2}\right)}+\frac{c_{z}}{2} \\
& +\frac{\left(\gamma_{p} A_{3}-A_{1} \theta_{p}\right) s_{m}-\left[A_{1}\left(\theta_{c}+\theta_{p}\right)+\gamma_{p} \theta_{c}\right] s_{r}+\left(A_{1} A_{4}-\gamma_{p} \theta_{p}\right) s_{z}+\left(A_{1} \varphi_{1}-\gamma_{p} \varphi_{2}\right) \mu}{2\left(A_{1} A_{2}-\gamma_{p}^{2}\right)} .
\end{aligned}
$$

The definition of new notations $A_{1}, A_{2}, A_{3}$, and $A_{4}$ is given in the Appendix (refer to Proof of Proposition 1).

Proposition 5. $\Pi_{r}$ is concave in $p_{r}$ under the given service levels provided that

$$
\gamma_{c}>\frac{6 \gamma_{c} \gamma_{p}\left(\gamma_{c}+\gamma_{p}\right)+3 \gamma_{c}^{2} A_{2}+3\left(\gamma_{c}+\gamma_{p}\right)^{2} A_{1}}{4\left(A_{1} A_{2}-\gamma_{p}^{2}\right)}-A_{1}
$$

$$
p_{r}^{*}=\frac{1}{2} c_{s}-\frac{L_{1}+L_{2} s_{r}+L_{3} s_{m}+L_{4} s_{z}+L_{5} \mu}{2 L_{6}} .
$$

The definition of new notations $L_{1}, L_{2}, L_{3}, L_{4}, L_{5}$, and $L_{6}$ is given in the Appendix, Proof of Proposition 5.

Proposition 6. $\Pi_{r}$ is concave in $s_{r}$ provided that $\eta_{2}>-\left(L_{2} M_{2} / 2 L_{6}\right)$. The unique optimal service level is derived as follows:

The unique optimal $p_{r}$ is derived as follows:

$$
s_{r}^{*}=-\frac{L_{2} M_{1}+L_{1} M_{2}+c_{s} L_{6} M_{2}}{4 \eta_{2} L_{6}+2 L_{2} M_{2}}-\frac{\left(L_{2} M_{3}+L_{3} M_{2}\right) s_{m}+\left(L_{2} M_{4}+L_{4} M_{2}\right) s_{z}+\left(L_{2} M_{5}+L_{5} M_{2}\right) \mu}{4 \eta_{2} L_{6}+2 L_{2} M_{2}} .
$$

The definition of new notations $M_{1}, M_{2}, M_{3}, M_{4}$, and $M_{5}$ is given in the Appendix, Proof of Proposition 6.
Proposition 7. $\Pi_{m}$ is concave in $s_{m}, s_{z}$, and $\mu$ provided that $\mathrm{O}_{2}<0, \mathrm{O}_{2} P_{3}-\mathrm{O}_{3} P_{2}>0$ and $\mathrm{O}_{2}\left(P_{3} Q_{4}-P_{4} Q_{3}\right)+\mathrm{O}_{3}\left(P_{4} Q_{2}\right.$ $\left.-P_{2} Q_{4}\right)+O_{4}\left(P_{2} Q_{3}-P_{3} Q_{2}\right)<0$. The equilibrium decisions can be obtained by

$$
\begin{gathered}
s_{m}^{*}=\frac{O_{1}\left(P_{4} Q_{3}-P_{3} Q_{4}\right)+O_{3}\left(P_{1} Q_{4}-P_{4} Q_{1}\right)+O_{4}\left(P_{3} Q_{1}-P_{1} Q_{3}\right)}{O_{2}\left(P_{3} Q_{4}-P_{4} Q_{3}\right)+O_{3}\left(P_{4} Q_{2}-P_{2} Q_{4}\right)+O_{4}\left(P_{2} Q_{3}-P_{3} Q_{2}\right)}, \\
s_{z}^{*}=\frac{O_{1}\left(P_{2} Q_{4}-P_{4} Q_{2}\right)+O_{2}\left(P_{4} Q_{1}-P_{1} Q_{4}\right)+O_{4}\left(P_{1} Q_{2}-P_{2} Q_{1}\right)}{O_{2}\left(P_{3} Q_{4}-P_{4} Q_{3}\right)+O_{3}\left(P_{4} Q_{2}-P_{2} Q_{4}\right)+O_{4}\left(P_{2} Q_{3}-P_{3} Q_{2}\right)}, \\
\mu^{*}=\frac{O_{1}\left(P_{3} Q_{2}-P_{2} Q_{3}\right)+O_{3}\left(P_{1} Q_{3}-P_{3} Q_{1}\right)+O_{3}\left(P_{2} Q_{1}-P_{1} Q_{2}\right)}{O_{2}\left(P_{3} Q_{4}-P_{4} Q_{3}\right)+O_{3}\left(P_{4} Q_{2}-P_{2} Q_{4}\right)+O_{4}\left(P_{2} Q_{3}-P_{3} Q_{2}\right)} .
\end{gathered}
$$

The definition of new notations mentioned above, such as $O_{1}$, $P_{1}$, and $Q_{1}$, are given in the Appendix, Proof of Proposition 7.

4.3. Nash Game Model. In the Nash game model, the manufacturer and the retailer have almost the same bargaining power. They make their pricing decisions independently and simultaneously under the given service levels. The sequence of decision-making is as shown in Figure 2(c). First, the manufacturer determines the service level of the standard products sold via the online channel $s_{m}$, the service level of the customized products $s_{z}$, and the customization level $\mu$ to maximize 
his profits. Then the retailer sets the optimal service level for the standard products sold offline $s_{r}$. In the next stage, the retailer sets the optimal retail price of the standard products sold offline $p_{r}$ to maximize her profits. Simultaneously, the manufacturer sets the optimal wholesale price of the standard products $w$, the optimal direct sales price of the standard products $p_{m}$, and the optimal price of the customized products $p_{z}$ to maximize his profit. Backward deduction is applied to solve the problem.

Proposition 8. $\Pi_{m}$ is jointly concave in $p_{m}$ and $p_{z}$ under the given service levels and customization level. The retailer's profit is concave in $p_{r}$. The optimal solutions can be derived as follows:

$$
\begin{gathered}
p_{r}^{*}=\frac{R_{1}+R_{2} s_{r}+R_{3} s_{m}+R_{4} s_{z}+R_{5} \mu}{R_{6}}, \\
p_{m}^{*}=\frac{T_{1}+T_{2} s_{r}+T_{3} s_{m}+T_{4} s_{z}+T_{5} \mu}{R_{6}}, \\
p_{z}^{*}=\frac{U_{1}+U_{2} s_{r}+U_{3} s_{m}+U_{4} s_{z}+U_{5} \mu}{R_{6}} .
\end{gathered}
$$

$$
\begin{gathered}
s_{m}^{*}=\frac{V_{1}\left(X_{4} Y_{3}-X_{3} Y_{4}\right)+V_{3}\left(X_{1} Y_{4}-X_{4} Y_{1}\right)+V_{4}\left(X_{3} Y_{1}-X_{1} Y_{3}\right)}{V_{2}\left(X_{3} Y_{4}-X_{4} Y_{3}\right)+V_{3}\left(X_{4} Y_{2}-X_{2} Y_{4}\right)+V_{4}\left(X_{2} Y_{3}-X_{3} Y_{2}\right)} \\
s_{z}^{*}=\frac{V_{1}\left(X_{2} Y_{4}-X_{4} Y_{2}\right)+V_{2}\left(X_{4} Y_{1}-X_{1} Y_{4}\right)+V_{4}\left(X_{1} Y_{2}-X_{2} Y_{1}\right)}{V_{2}\left(X_{3} Y_{4}-X_{4} Y_{3}\right)+V_{3}\left(X_{4} Y_{2}-X_{2} Y_{4}\right)+V_{4}\left(X_{2} Y_{3}-X_{3} Y_{2}\right)}, \\
\mu^{*}=\frac{V_{1}\left(X_{3} Y_{2}-X_{2} Y_{3}\right)+V_{2}\left(X_{1} Y_{3}-X_{3} Y_{1}\right)+V_{3}\left(X_{2} Y_{1}-X_{1} Y_{2}\right)}{V_{2}\left(X_{3} Y_{4}-X_{4} Y_{3}\right)+V_{3}\left(X_{4} Y_{2}-X_{2} Y_{4}\right)+V_{4}\left(X_{2} Y_{3}-X_{3} Y_{2}\right)} .
\end{gathered}
$$

The definition of new notations mentioned above, such as $V_{1}, X_{1}$, and $Y_{1}$, is given in the Appendix, Proof of Proposition 10.

\section{Coordination of the MS Model}

This section investigates the coordination of the MS model. In the above three models, both price competition and service competition exist between different channels and between different products. In this coordination model, the retailer is assumed to provide the same service for different products to eliminate the service competition among channels and products. Additionally, the manufacturer shares some service costs at the ratio $\varphi_{0}$. The decisionmaking sequence is presented in Figure 3. The manufacturer first determines the optimal customization level $\mu$, and then, the retailer sets the service level $s$ for all products. Next, the manufacturer determines the wholesale price and the direct sales price of the standard products and the price of the customized products. In reaction to the former decisions, the retailer chooses the optimal retail price of the standard products to maximize her own profits.

The demand function of the standard products sold via the online channel is $D_{m}=\alpha_{m}+\gamma_{c} p_{r}-A_{1} p_{m}+\gamma_{p} p_{z}+$ $\beta_{s} s-\varphi_{2} \mu$, the demand function of the standard products sold via the offline channel is $D_{r}=\alpha_{r}-\left(A_{1}+\gamma_{c}\right) p_{r}+\gamma_{c} p_{m}+$ $\left(\gamma_{c}+\gamma_{p}\right) p_{z}+\beta_{s} s-\varphi_{2} \mu$, and the demand function of the customized products is $D_{z}=\alpha_{z}+\left(\gamma_{c}+\gamma_{p}\right) p_{r}+\gamma_{p} p_{m}-$ $A_{2} p_{z}+\beta_{z} s+\varphi_{1} \mu$. Based on the demand functions above, the profit functions $\Pi_{m}$ and $\Pi_{r}$ are obtained as follows:

$$
\begin{aligned}
& \Pi_{m}=\left(w-c_{s}\right) D_{r}+\left(p_{m}-c_{s}\right) D_{m}+\left(p_{z}-c_{z}\right) D_{z}-\frac{1}{2} \varphi_{0} \eta_{2} s^{2}-\frac{1}{2} C \mu^{2} \\
& \Pi_{r}=\left(p_{r}-w\right) D_{r}-\frac{1}{2}\left(1-\varphi_{0}\right) \eta_{2} s^{2} .
\end{aligned}
$$




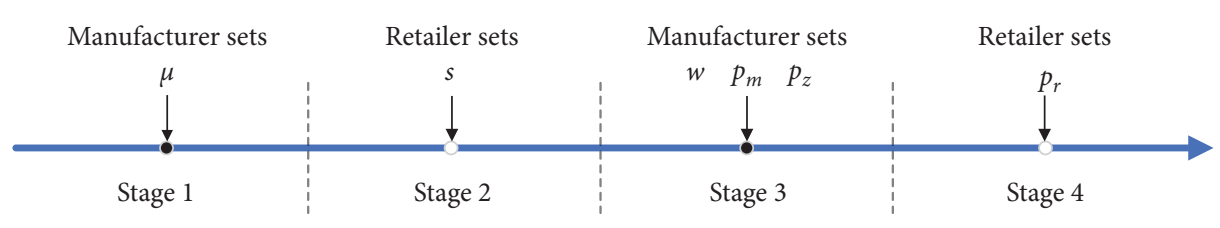

FIGURE 3: Decision-making sequences in the coordination model.

Under the conditions in Proposition 1, the optimal pricing decisions are as follows:

$$
\begin{aligned}
w^{*}= & \frac{B_{1}+G_{1} \mu+\left\{1 / 2 \beta_{s} A_{5}-2\left[\gamma_{c} A_{2}+\gamma_{p}\left(\gamma_{c}+\gamma_{p}\right)\right]\left[\gamma_{c} \beta_{s} / 2\left(A_{1}+\gamma_{c}\right)+\beta_{s}\right]\right\} s}{A_{6}} \\
& -\frac{2\left[A_{1}\left(\gamma_{c}+\gamma_{p}\right)+\gamma_{c} \gamma_{p}\right]\left[\left(\gamma_{c}+\gamma_{p}\right) \beta_{s} / 2\left(A_{1}+\gamma_{c}\right)+\beta_{z}\right] s}{A_{6}}, \\
p_{m}^{*}= & \frac{B_{2}+G_{2} \mu-2\left[\gamma_{c}\left(\gamma_{c}+\gamma_{p}\right)+\gamma_{p}\left(A_{1}+\gamma_{c}\right)\right]\left[\left(\gamma_{c}+\gamma_{p}\right) \beta_{s} / 2\left(A_{1}+\gamma_{c}\right)+\beta_{z}\right] s}{A_{6}} \\
& -\frac{2\left[\left(\gamma_{c}+\gamma_{p}\right)^{2}-\left(A_{1}+\gamma_{c}\right) A_{2}\right]\left[\gamma_{c} \beta_{s} / 2\left(A_{1}+\gamma_{c}\right)+\beta_{s}\right]+\beta_{s}\left[\gamma_{c} A_{2}+\gamma_{p}\left(\gamma_{c}+\gamma_{p}\right)\right]}{A_{6}} s, \\
p_{z}^{*}= & \frac{B_{3}+G_{3} \mu-2\left[\gamma_{c}\left(\gamma_{c}+\gamma_{p}\right)+\gamma_{p}\left(A_{1}+\gamma_{c}\right)\right]\left[\gamma_{c} \beta_{s} / 2\left(A_{1}+\gamma_{c}\right)+\beta_{s}\right] s}{A_{6}} \\
& +\frac{2\left[\gamma_{c}^{2}-\left(A_{1}+\gamma_{c}\right) A_{1}\right]\left[\left(\gamma_{c}+\gamma_{p}\right) \beta_{s} / 2\left(A_{1}+\gamma_{c}\right)+\beta_{z}\right]-\beta_{s}\left[A_{1}\left(\gamma_{c}+\gamma_{p}\right)+\gamma_{c} \gamma_{p}\right]}{A_{6}} s, \\
p_{r}^{*}= & \frac{\alpha_{r}+\gamma_{c} p_{m}+\left(\gamma_{c}+\gamma_{p}\right) p_{z}+\beta_{s} s-\varphi_{2} \mu}{2\left(\delta_{s}+2 \gamma_{c}+\gamma_{p}\right)}+\frac{w}{2} .
\end{aligned}
$$

Proposition 11. $\Pi_{r}$ is concave in $s_{r}$ provided that $\left(1-\varphi_{0}\right) \eta_{2}-2 \Theta_{1} \Theta_{2}>0$. The unique optimal service level is derived as follows:

$$
\begin{aligned}
s^{*}= & \frac{\left[\alpha_{r}+\gamma_{c} B_{2}+\left(\gamma_{c}+\gamma_{p}\right) B_{3}-\left(A_{1}+\gamma_{c}\right) B_{4} / A_{6}\right] \Theta_{1}+B_{4}-B_{1} / A_{6} \Theta_{2}}{\left(1-\varphi_{0}\right) \eta_{2}-2 \Theta_{1} \Theta_{2}} \\
& +\frac{\left[\gamma_{c} G_{2}+\left(\gamma_{c}+\gamma_{p}\right) G_{3}-\left(A_{1}+\gamma_{c}\right) G_{4}-\varphi_{2} A_{6}\right] \Theta_{1}+\left(G_{4}-G_{1}\right) \Theta_{2}}{A_{6}\left[\left(1-\varphi_{0}\right) \eta_{2}-2 \Theta_{1} \Theta_{2}\right]} \mu .
\end{aligned}
$$

The definition of new notations $\Theta_{1}$ and $\Theta_{2}$ is given in the Appendix, Proof of Proposition 11.

$$
\begin{aligned}
C & +\varphi_{0} \eta_{2} Z_{10}^{2}-2 Z_{2}\left[-\left(A_{1}+\gamma_{c}\right) Z_{8}+\gamma_{c} Z_{4}+\left(\gamma_{c}+\gamma_{p}\right) Z_{6}+\beta_{s} Z_{10}-\varphi_{2}\right] \\
& -2 Z_{4}\left(\gamma_{c} Z_{8}-A_{1} Z_{4}+\gamma_{p} Z_{6}+\beta_{s} Z_{10}-\varphi_{2}\right)-2 Z_{6}\left[\left(\gamma_{c}+\gamma_{p}\right) Z_{8}+\gamma_{p} Z_{4}-A_{2} Z_{6}+\beta_{z} Z_{10}+\varphi_{1}\right]>0 .
\end{aligned}
$$


The unique optimal customization level is derived as follows:

$$
\begin{aligned}
\mu^{*}= & \frac{\left(Z_{1}-c_{s}\right)\left[-\left(A_{1}+\gamma_{c}\right) Z_{8}+\gamma_{c} Z_{4}+\left(\gamma_{c}+\gamma_{p}\right) Z_{6}+\beta_{s} Z_{10}-\varphi_{2}\right]}{Z_{11}} \\
& +\frac{Z_{2}\left[\alpha_{r}-\left(A_{1}+\gamma_{c}\right) Z_{7}+\gamma_{c} Z_{3}+\left(\gamma_{c}+\gamma_{p}\right) Z_{5}+\beta_{s} Z_{9}\right]}{Z_{11}} \\
& +\frac{\left(Z_{5}-c_{z}\right)\left[\left(\gamma_{c}+\gamma_{p}\right) Z_{8}+\gamma_{p} Z_{4}-A_{2} Z_{6}+\beta_{z} Z_{10}+\varphi_{1}\right]}{Z_{11}} \\
& +\frac{Z_{6}\left[\alpha_{z}+\left(\gamma_{c}+\gamma_{p}\right) Z_{7}+\gamma_{p} Z_{3}-A_{2} Z_{5}+\beta_{s} Z_{9}\right]}{Z_{11}} \\
& +\frac{\left(Z_{3}-c_{s}\right)\left(\gamma_{c} Z_{8}-A_{1} Z_{4}+\gamma_{p} Z_{6}+\beta_{s} Z_{10}-\varphi_{2}\right)}{Z_{11}} \\
& +\frac{Z_{4}\left(\alpha_{m}+\gamma_{c} Z_{7}-A_{1} Z_{3}+\gamma_{p} Z_{5}+\beta_{s} Z_{9}\right)-\varphi_{0} \eta_{2} Z_{9} Z_{10}}{Z_{11}} .
\end{aligned}
$$

The definition of new notations, such as $Z_{1}$ and $Z_{2}$, is given in the Appendix, Proof of Proposition 12.

Next, the MS model and coordination model are compared in terms of the profits of all members to analyze whether the service-cost-sharing contract is effective, as in Figure 4. Under the premise that the retailer is profitable, the retailer's profits in the MS model are always higher than those in the coordination model as the service-cost-sharing ratio $\varphi_{0}$ changes. Both the manufacturer's profits and the overall supply chain profits in the coordination model are higher than those in the MS model when the retailer is profitable.

In summary, the service-cost-sharing contract can coordinate the dual-channel supply chain with product customization when the service-cost-sharing ratio $\varphi_{0}$ is appropriate. Therefore, for decision-makers in the supply chain, it is vital to integrate service providers to achieve economies of scale and share service costs to ease the negative effects resulting from the service competition.

\section{Numerical Studies}

6.1. Sensitivity Analysis. In this section, the impacts of different parameters $\left(\gamma_{c}, \gamma_{p}, \theta_{c}, \theta_{p}, \eta_{1}, \eta_{2}\right.$, and $\left.\varphi_{1}\right)$ on the profits, price decisions, and service decisions of all members are studied. First, both the price and service competition coefficients are varied to examine the impacts of the price and service competition intensity on SC members' equilibrium profits, correspondingly. Second, the effects of the service cost coefficients $\eta_{1}$ and $\eta_{2}$ on price and service decisions are investigated. Finally, the effects of customers' sensitivity to the customization level on price and service decisions are analyzed. The basic concerning parameters are set as follows: $\alpha_{m}=150, \alpha_{r}=300, \alpha_{z}=150, \delta_{s}=0.8$, $\delta_{z}=0.6, \beta_{s}=0.5$, and $\beta_{z}=0.3$. Table 1 summarizes the change of the optimal decisions or profits with the changing of the seven parameters in the MS model, RS model, and Nash game model. In Table 1, the notations are defined as follows: + : monotonically increasing; - : monotonically decreasing; $\mathrm{N}$ : unclear effect by the corresponding parameters. For example, “+” means the optimal direct sales price $p_{m}$ increases with the price competition coefficients between online and offline channels $\gamma_{c}$ in the MS model; “-” means the optimal wholesale price $w$ decreases with the service competition between online and offline channels in the MS model; "N" means the variation of customers sensitivity to customization barely has any impact on the standard products' service level provided by the retailer.

6.1.1. Effects of Price Competition on Optimal Decisions. This section examines the effects of price competition on the equilibrium decisions and profits in the three models, as in Table 1. The optimal $w, p_{r}$, and $p_{z}$ all decrease as the price competition increases in the three models. Obviously, all supply chain members will reduce their product prices according to the product prices set by their competitors when the price competition intensifies. The standard products sold via the retail channel only compete with the standard products sold online, and the customized products sold online also compete with the standard products sold online. Therefore, the prices $w, p_{r}$, and $p_{z}$ will decrease. In contrast, the standard products sold directly compete with both the standard products sold via the retail channel and the customized products. For the manufacturer, his final goal is to increase his sales volume and enlarge his market share. Under the existence of crossproduct competition and cross-channel competition, the 


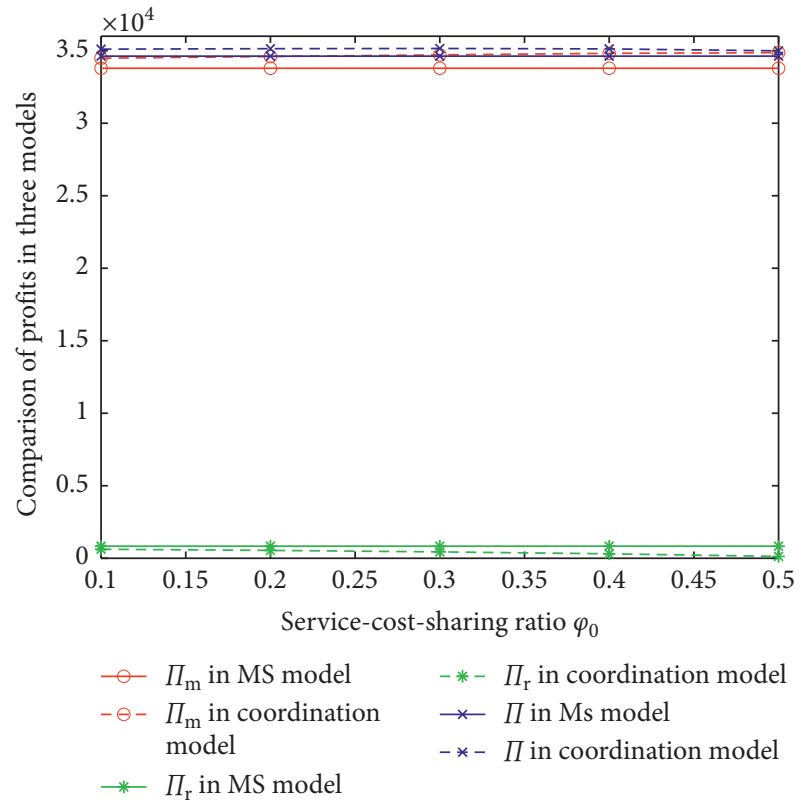

Figure 4: Comparison of MS model and coordination model in profits.

TABLE 1: The sensitivity analysis for seven parameters.

\begin{tabular}{|c|c|c|c|c|c|c|c|c|}
\hline & & $\gamma_{c}$ & $\gamma_{p}$ & $\theta_{c}$ & $\theta_{p}$ & $\eta_{1}$ & $\eta_{2}$ & $\varphi_{1}$ \\
\hline \multirow{11}{*}{ MS } & $w$ & - & - & - & - & + & - & + \\
\hline & $p_{r}$ & - & - & - & - & + & - & + \\
\hline & $p_{m}$ & + & + & - & - & + & + & + \\
\hline & $p_{z}$ & - & - & + & + & - & + & + \\
\hline & $s_{r}$ & - & - & + & + & + & - & - \\
\hline & $s_{m}$ & + & + & - & - & - & + & - \\
\hline & $s_{z}$ & - & - & + & + & - & + & + \\
\hline & $\mu$ & - & - & + & + & - & + & + \\
\hline & $\Pi_{m}$ & + & + & + & + & - & - & + \\
\hline & $\Pi_{r}$ & - & - & - & - & + & - & - \\
\hline & $\Pi$ & + & + & - & - & - & - & + \\
\hline \multirow{11}{*}{$\mathrm{RS}$} & $w$ & - & - & + & + & + & - & - \\
\hline & $p_{r}$ & - & - & + & + & + & - & - \\
\hline & $p_{m}$ & - & - & + & + & - & + & - \\
\hline & $p_{z}$ & - & - & - & - & - & + & + \\
\hline & $s_{r}$ & - & - & + & + & + & - & $\mathrm{N}$ \\
\hline & $s_{m}$ & - & + & + & + & - & + & + \\
\hline & $s_{z}$ & - & - & - & - & - & + & + \\
\hline & $\mu$ & - & - & + & + & - & + & + \\
\hline & $\Pi_{m}$ & - & - & + & + & - & - & + \\
\hline & $\Pi_{r}$ & - & - & + & + & + & - & - \\
\hline & $\Pi$ & - & - & + & + & + & - & - \\
\hline \multirow{11}{*}{ Nash } & $w$ & - & - & + & + & + & - & - \\
\hline & $p_{r}$ & - & - & + & + & + & - & - \\
\hline & $p_{m}$ & - & - & + & + & - & + & - \\
\hline & $p_{z}$ & - & - & - & - & - & + & + \\
\hline & $s_{r}$ & - & - & + & + & + & - & $\mathrm{N}$ \\
\hline & $s_{m}$ & - & + & + & + & - & + & - \\
\hline & $s_{z}$ & - & - & - & - & - & + & + \\
\hline & $\mu$ & - & - & - & - & - & + & + \\
\hline & $\Pi_{m}$ & - & - & - & - & - & + & + \\
\hline & $\Pi_{r}$ & - & - & + & + & + & - & - \\
\hline & $\Pi$ & - & - & + & + & + & - & + \\
\hline
\end{tabular}

manufacturer is the dominant player with more bargaining power in the MS model. He tends to increase $p_{m}$ to earn more profit and reduce $w$ to maintain his sales volume, which does not work in the RS model and Nash model. This also explains why the manufacturer would provide better service for the standard products sold online. Furthermore, the price competition between the online and offline channels has less impact on the service level of the standard products sold online. Moreover, the intense price competition makes it difficult for all members to provide better service for other products.

The customization level $\mu$ provided by the manufacturer decreases as the price competition between the two channels $\gamma_{c}$ and between different products $\gamma_{p}$ increase in all three models. This indicates that price competition discourages the manufacturer from providing customized products.

In the MS model, the manufacturer with more bargaining power will gain more profits with price competition among channels and among products, as will the overall supply chain. However, in the RS model and the Nash model, the profits of both parties decrease.

6.1.2. Effects of Service Competition on Optimal Decisions. This section examines the effects of service competition on the equilibrium decisions and profits in the three models (as in Table 1).

In the RS model and Nash model, the optimal $w, p_{r}$, and $p_{z}$ increase as the service competition coefficients $\theta_{c}$ and $\theta_{p}$ increase. This is because service competition leads to decreased of demand. The retailer in the RS model and the Nash model has more bargaining power than that in the MS model, so it tends to set a higher price for standard products to acquire more profits. In reaction, the manufacturer will increase $w$ while lowering $p_{m}$ to promote the online sales of the standard products. Nevertheless, in the MS model, the retailer's bargaining power is weaker than that of the manufacturer, which leads to the retailer lowering prices as the service competition intensifies. With the more intense service competition among different products or different channels, the demand for the standard products sold at retail decreases the most. This is why the retailer must lower the retail price for standard products.

The optimal service level for standard products sold offline $s_{r}$ increases as the service competition coefficients $\theta_{c}$ and $\theta_{p}$ increase in the MS model while the service levels provided by the manufacturer for the standard products $s_{m}$ and for the customized products $s_{z}$ both decrease. In the RS model and the Nash model, the service levels for the standard products $s_{r}$ and $s_{m}$ increase as $\theta_{c}$ and $\theta_{p}$ increase while the service level for the customized products $s_{z}$ decreases.

In the MS model and RS model, the customization level $\mu$ increases as the service competition coefficients $\theta_{c}$ and $\theta_{p}$ increase, and in the Nash model, the customization level $\mu$ decreases as $\theta_{c}$ and $\theta_{p}$ increases. In other words, for the manufacturer, providing a higher customization level will help him stand out in the service competition and obtain more profits. 
In terms of profits, $\Pi_{m}$ increases and $\Pi_{r}$ and $\Pi$ decrease as $\theta_{c}$ and $\theta_{p}$ increase in the MS model. In the RS model, the profits of both members increase as the service competition increases. However, $\Pi_{m}$ decreases as the service competition coefficients increase. In general, service competition is beneficial for the overall supply chain under the retailer Stackelberg and Nash games.

\subsubsection{Effects of Service Cost Coefficients on Optimal Decisions.} This section examines the effects of service cost coefficients on the equilibrium decisions and profits in the three models (as in Table 1).

As $\eta_{1}$ increases, the optimal $w$ and $p_{r}$ increase. In addition, the optimal $p_{m}$ increases as $\eta_{1}$ increases in the MS model but it decreases as $\eta_{1}$ increases in the RS model and Nash model. In addition, the optimal price for the customized products $p_{z}$ decreases as $\eta_{1}$ increases in all three models. As $\eta_{2}$ increases, the optimal $w$ and $p_{r}$ decrease. Furthermore, the optimal $p_{m}$ and $p_{z}$ increase as $\eta_{2}$ increases. The increased $\eta_{2}$ leads to a higher unit service cost for the retailer. She will lower the service level for the standard products to control the total service costs. Moreover, the manufacturer will provide a high-level service for his products and improve the customization level, as in Table 1.

Moreover, the optimal $s_{r}$ increases as $\eta_{1}$ increases in the three models. However, $s_{m}$ and $s_{z}$ both decrease as $\eta_{1}$ increases, which is opposite when the service cost coefficient $\eta_{2}$ changes. The impacts of the service cost coefficient on service levels show that the service level provided by the supply chain members has a negative correlation with the service cost coefficients.

Under the Stackelberg game, both the manufacturer and the retailer will not benefit from increasing their own service cost coefficients. In general, increased service cost coefficients are beneficial for the overall supply chain in the RS model and the Nash model.

6.1.4. Effects of Customer Sensitivity Coefficient to the Customization Level on Optimal Decisions. As in Table 1, the increase in the customer sensitivity coefficient to the customization level $\varphi_{1}$ leads to an increase in all products' prices in the MS model. In addition, the optimal $w, p_{r}$, and $p_{z}$ all decrease as the customer sensitivity coefficient to the customization level $\varphi_{1}$ increases, but the price of the customized products increases. Apparently, the increase in $\varphi_{1}$ leads to a higher demand for the customized products, so the manufacturer can increase the price to acquire more profits, which also explains the increasing profits of the manufacturer. Otherwise, the retailer's profits will decrease, even if she tries to enhance the service level, because as the customer sensitivity coefficient to the customization level increases, the impacts of the service competition on decisions are weakened.

In summary, the increase in the customer sensitivity coefficient to the customization level is beneficial for the manufacturer and is harmful to the retailer, and the impact on the overall supply chain depends on the supply chain structure.
6.1.5. Managerial Insights. Through the sensitivity analysis of some parameters $\left(\gamma_{c}, \gamma_{p}, \theta_{c}, \theta_{p}, \eta_{1}, \eta_{2}\right.$, and $\left.\varphi_{1}\right)$ on the profits, the optimal price, and service decisions, some managerial insights can be concluded.

First, when the price competition is fierce, the manufacturer should not lower the product price blindly, but rather should price and provide services for the standard products and customized products differently to avoid channel competition for the standard products and enlarge the market for the customized products. Furthermore, as price competition intensifies, the manufacturer should focus on the marketing strategies of the standard products but lower the customization level.

Second, under service competition, the retailer should provide better service for the standard products sold at retail regardless of her bargaining power. When the service competition becomes fiercer, the manufacturer should choose to provide better service for the products he sells according to his status in the supply chain. For example, when the manufacturer does not lead the market, lowering the service level for the customized products can help the manufacturer reduce service expenditures and obtain more revenue. Moreover, when the bargaining power of the manufacturer and the retailer is asymmetric, the manufacturer should pay more attention to his customization construction. In addition, under the Nash scenario, considering service competition, customization expenditures should be reduced. In general, service competition is beneficial for both members when the retailer is the Stackelberg leader.

Third, when the service cost coefficient of one member increases, the member should reduce their service level. However, his competitor will optimize their service and obtain more market demand. Furthermore, when the manufacturer's service cost coefficient is high, the manufacturer should lower the service level for the customized products and the customization level to avoid competition with the standard products. In addition, he should focus on improving the service for the standard products sold online to retain his sales volume and optimize the performance of the supply chain. Besides, when the manufacturer does not lead the market, increasing his service cost coefficient is beneficial for the overall supply chain.

Finally, the customers are more sensitive to customization, which is more beneficial for the manufacturer to sell the customized products. In addition, he should invest more in customization construction and improve the customization level to provide more diversified customized products to increase his sales volume.

6.2. Comparison of Equilibrium Decisions and Profits in Three Models. This section compares the MS model, the RS model, and the Nash game model in terms of optimal prices, service levels, customization level, and profits of all members.

The optimal $w, p_{r}, p_{m}$, and $p_{z}$ are the highest in the MS model but the lowest in the Nash model, as in Figures 5-8. In the MS model, the manufacturer leads the market, and he tends to share more revenues by increasing the prices of both 


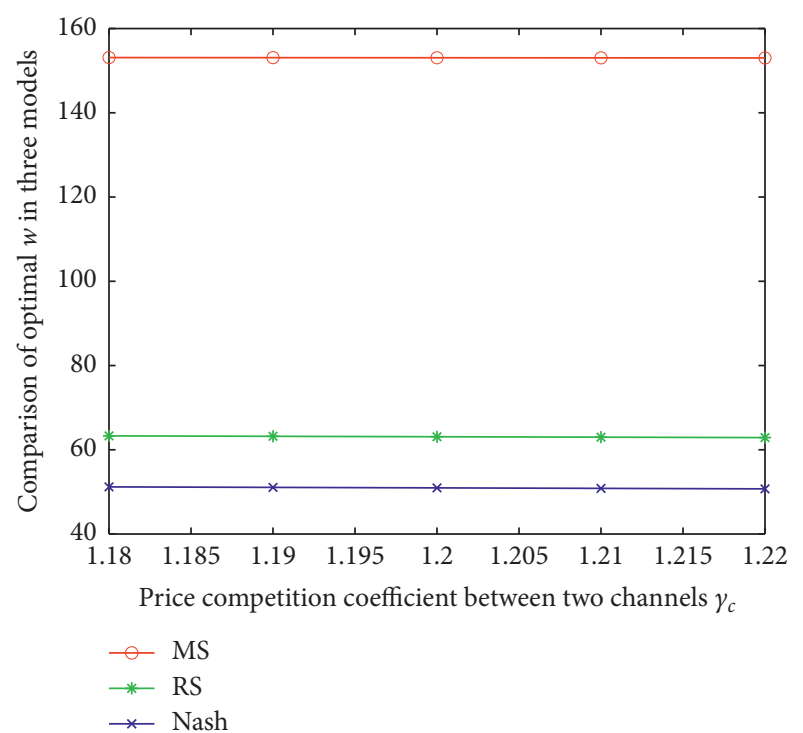

Figure 5: The comparison of the optimal wholesale price of standard products $w$ in the three models.

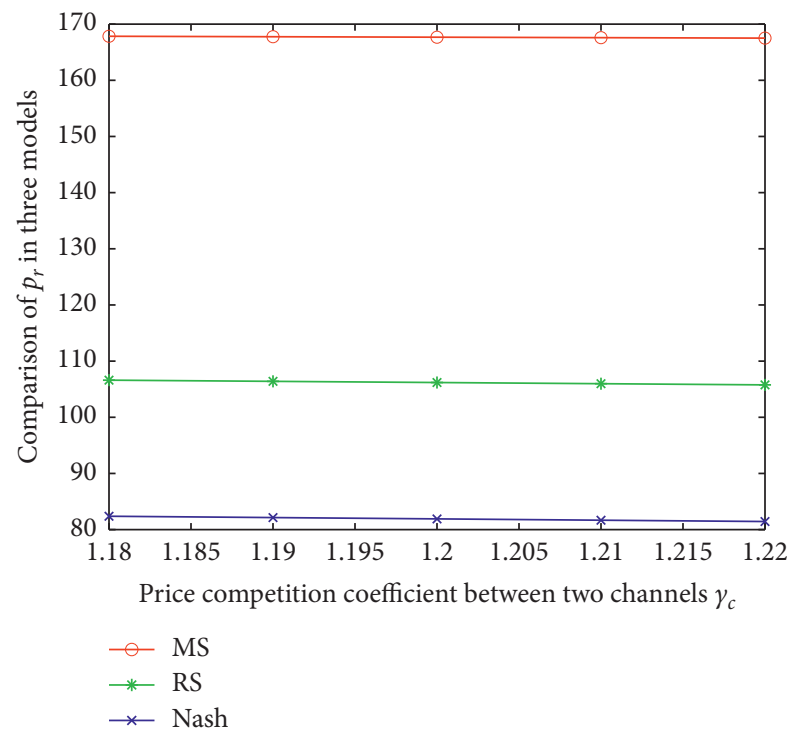

FIGURE 6: The comparison of the optimal retail price of standard products $p_{r}$ in the three models.

the standard products and customized products. Therefore, the retailer will increase $p_{r}$.

The retailer provides the highest service level for the standard products sold offline $s_{r}$ in the RS model among the three models but the lowest service level for the standard products sold offline $s_{r}$ in the MS model among the three models, as in Figure 9. In the RS model, the retailer has more control over the market and thus prefers to provide better service to gain more profits. Conversely, the retailer has the least control over the market in the MS model and tends to lower the service level to reduce unnecessary service costs. Similarly, the manufacturer provides the highest service level for the standard products sold via his direct sales channel $s_{m}$ in the RS model among the three models but the lowest

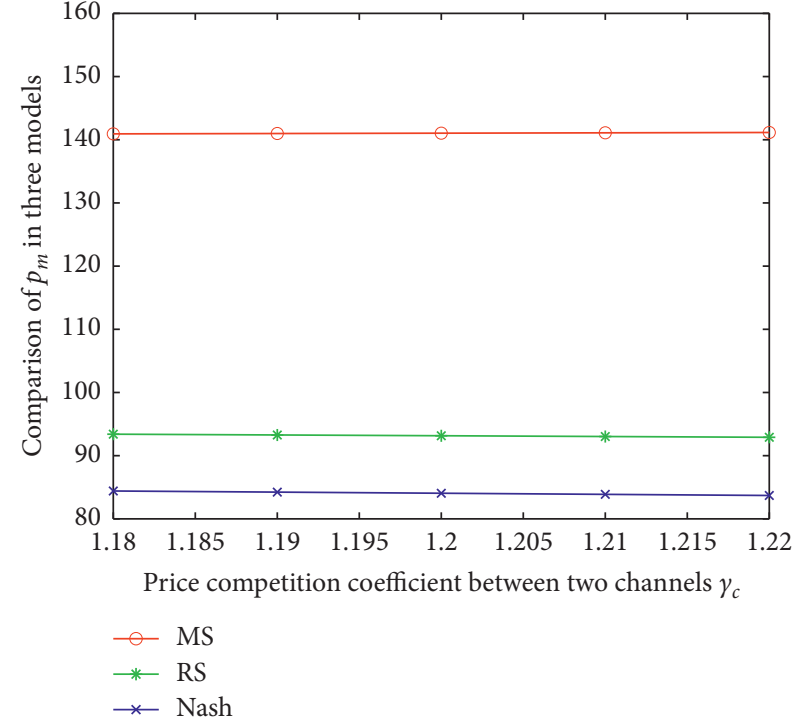

Figure 7: The comparison of the optimal direct sales price of standard products $p_{m}$ in the three models.

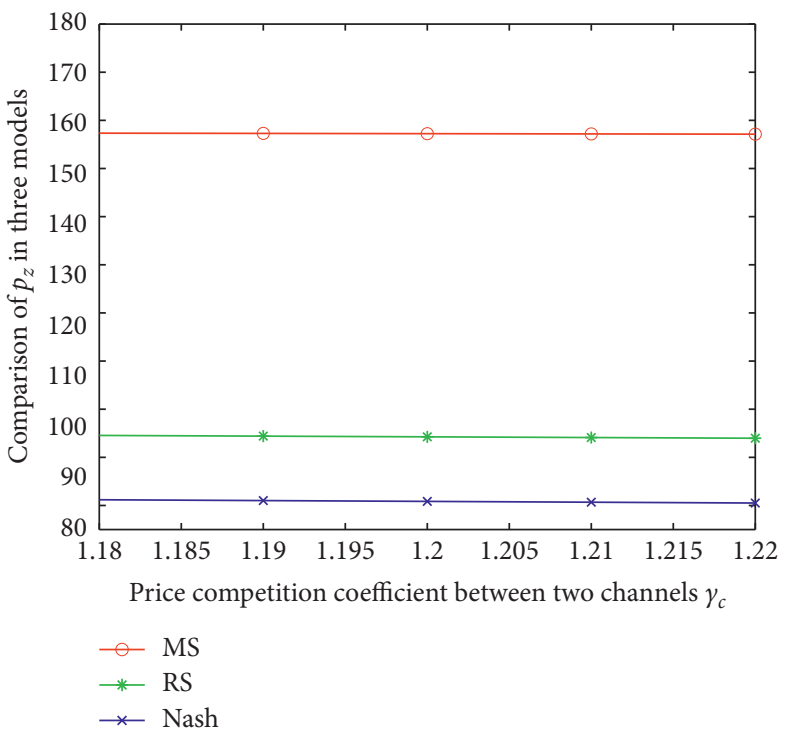

FIgURE 8: The comparison of the optimal direct sales price of customized products $p_{z}$ in the three models.

service level for the standard products sold via his direct sales channel $s_{m}$ in the MS model among the three models, as in Figure 10. In the MS model, the manufacturer has more control over the market, so it is necessary for the manufacturer to improve the service level. However, in the RS model, the manufacturer is led by the retailer's decision and tends to enhance the service level to acquire a greater market share. As in Figure 11, the manufacturer provides the highest service level $s_{z}$ for the customized products in the MS model but the lowest $s_{z}$ in the Nash model, which means that when the market is highly competitive and resources are limited, the manufacturer is more willing to maintain a high service level for the standard products to meet their market demand while compressing the service costs for the customized 


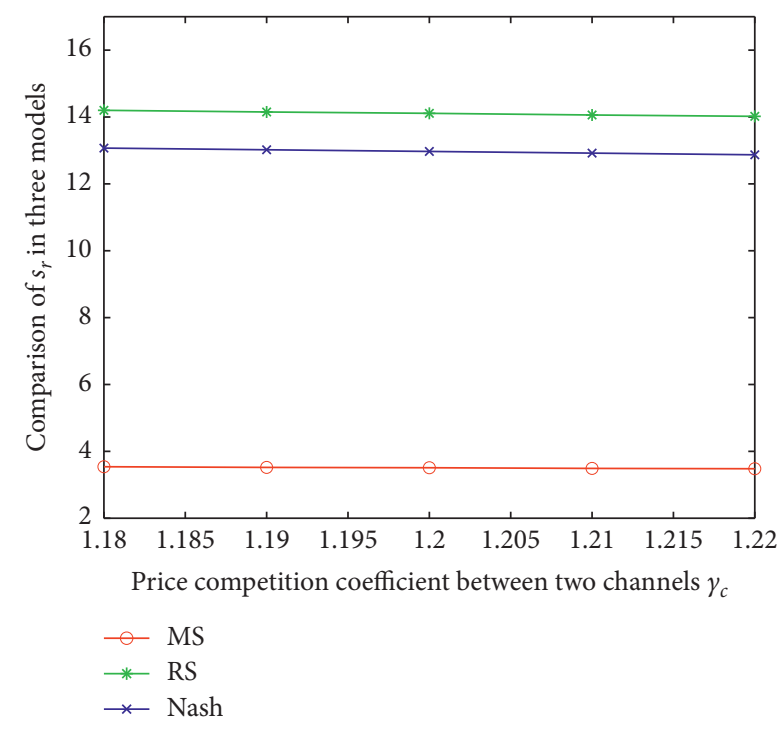

FIGURE 9: The comparison of the optimal service level of standard products in the retail channel $s_{r}$ in the three models.

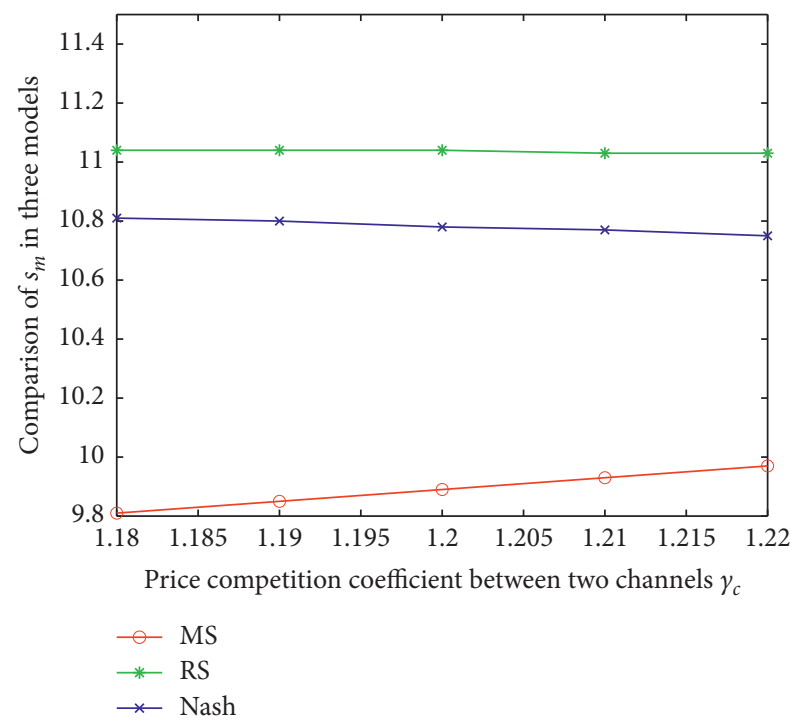

FIGURE 10: The comparison of the optimal service level of standard products in the direct sales channel $s_{m}$ in the three models.

products. Similarly, the customization level $\mu$ is the highest in the MS model but the lowest in the RS model, as in Figure 12.

As in Figure 13 and Figure 14, the manufacturer's profits and the overall supply chain's profits are both the highest in the MS model but the lowest in the Nash model. However, the retailer's profits are the highest in the RS model but the lowest in the MS model (in Figure 15). In the MS model, the manufacturer makes the most favorable decisions for him first, so the profits of the manufacturer are the highest in the MS model among the three models. Similarly, the retailer earns the most profits in the RS model due to her market leadership. The lowest profits for the manufacturer and the supply chain existing in the Nash model among the three

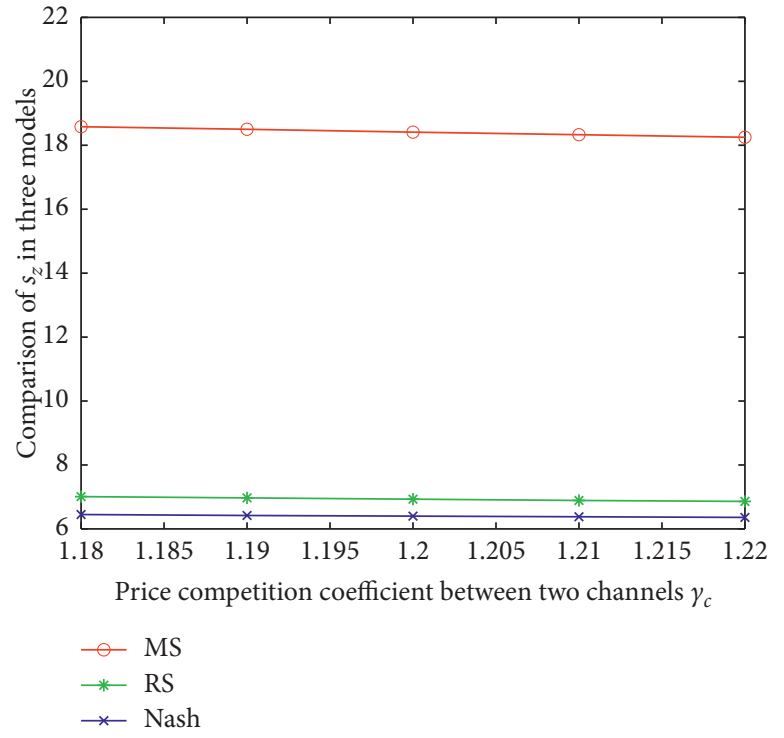

Figure 11: The comparison of the optimal service level of customized products $s_{z}$ in the three models.

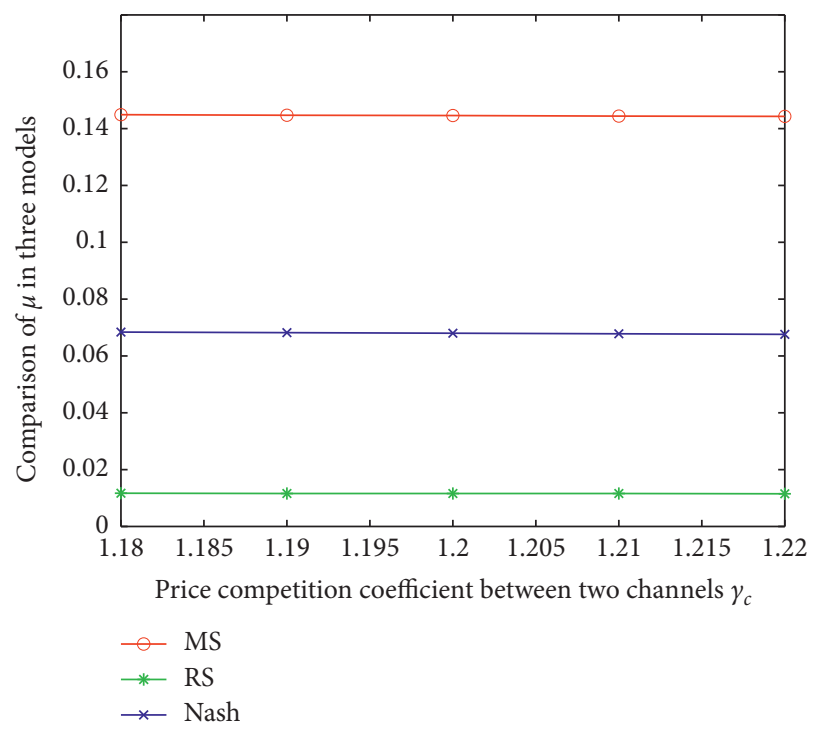

Figure 12: The comparison of the optimal customization level $\mu$ in the three models.

models indicate that the Nash model performs the worst. However, the MS model brings the most profits for the manufacturer and the supply chain, which means that it performs the best overall.

6.2.1. Managerial Insights. By comparing the three models, we obtain some managerial insights.

First, the prices of all products in the supply chain should be set higher when the manufacturer leads the market. When the manufacturer and the retailer are under the Nash scenario, product prices will decrease. The trend of the price changes is consistent with the overall supply chain revenues. 


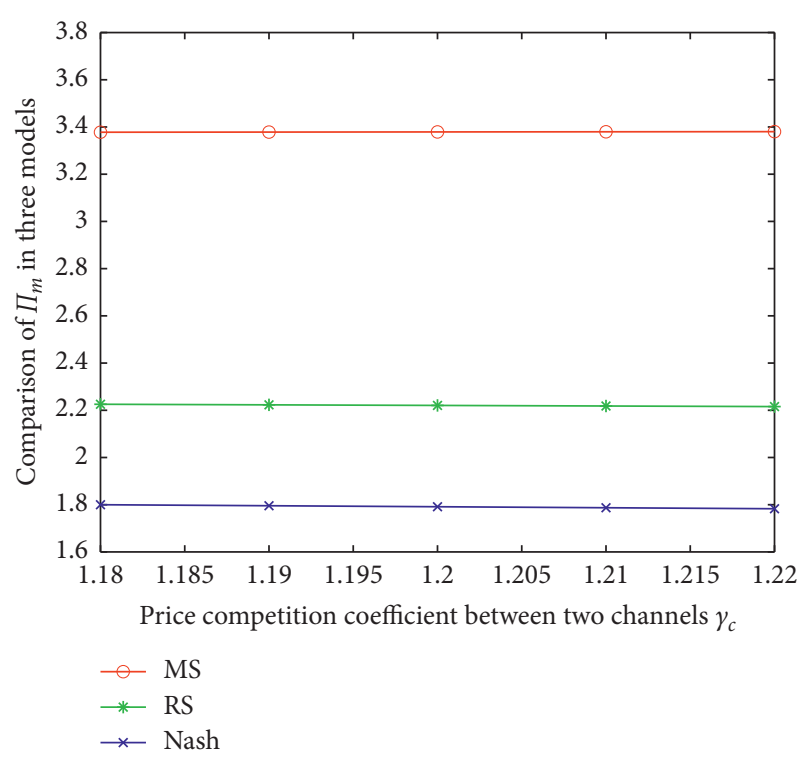

FIGURE 13: The comparison of the manufacturer's profit $\Pi_{m}$ in the three models.

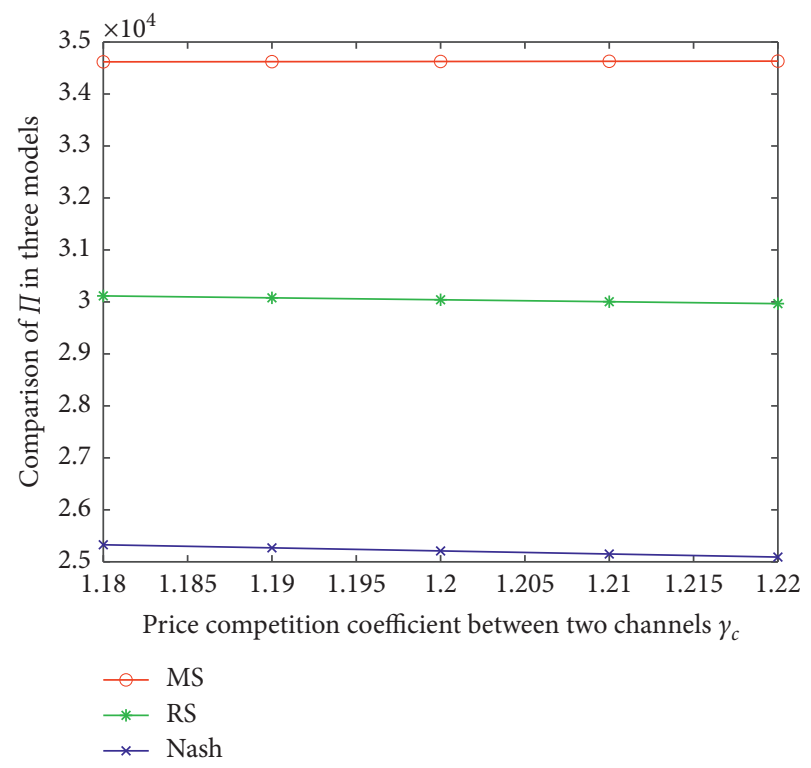

FIGURE 14: The comparison of the overall supply chain's profit $\Pi$ in the three models.

Second, when the manufacturer leads the market, he is encouraged to sell customized products and provide better service for the customized products. In addition, under the retailer Stackelberg, the manufacturer should focus on enhancing the service for the standard products.

Third, the revenue gap between the manufacturer and the retailer is the largest in the MS model among the three models. Therefore, the MS model desperately needs to be coordinated. Moreover, the decision-makers need to draw coordination contracts to ease the price and service competition while balancing the revenues of all parties.

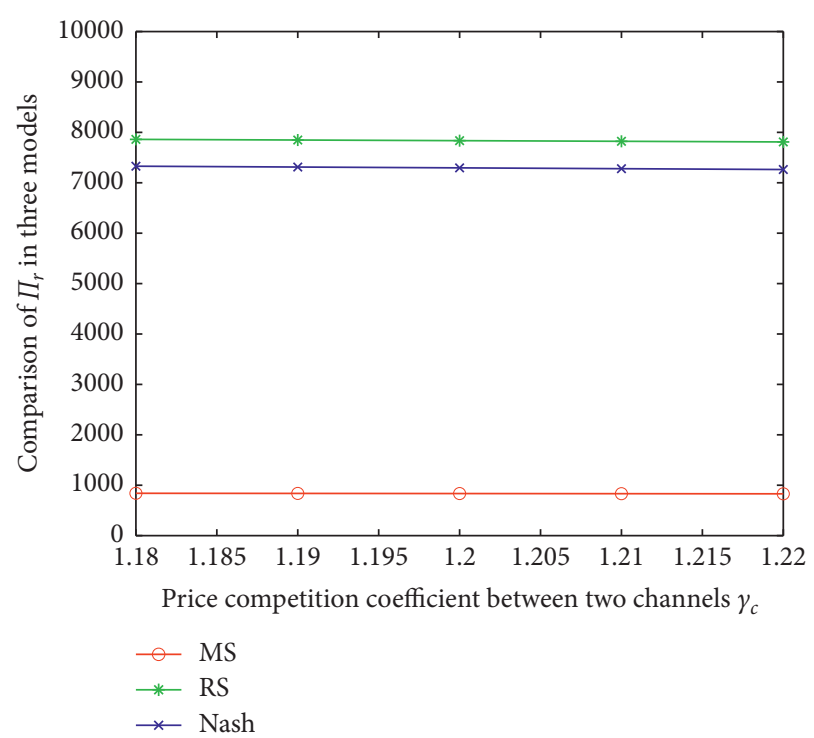

Figure 15: The comparison of the retailer's profit $\Pi_{r}$ in the three models.

\section{Conclusions}

The optimal decision of SC members under price and service competition is one of the major concerns and issues in supply chain management. In this paper, the dual-channel SC structure is comprised of one manufacturer and one retailer. Two kinds of products are sold in the supply chain: one is standard products, and the other is customized products. The manufacturer wholesales standard products to the retailer and sells both standard products and customized products directly through an online channel. The retailer earns profits by selling standard products. The customers' preference for customized products partially depends on the customization level provided by the manufacturer, which also affects the costs of customization construction. Additionally, the manufacturer and the retailer each provide services for the products they sell, including pre-sales and after-sales services. According to the supply chain structure described above, three power structure models are proposed and compared: (1) the MS model, (2) the RS model, and (3) the Nash game model. A service-cost-sharing contract is developed to coordinate the MS model. Then, the effects of customer sensitivity to customization, service costs, price competition, and service competition on the optimal prices, service levels, customization level, and all members' profits in all three models are analyzed.

Based on the numerical analysis, some managerial insights are offered. First, in a dual-channel SC with product customization, the manufacturer should lower the price of standard products but improve the price of customized products to avoid fierce price competition between different products and occupy both product markets. Second, retail services are a major advantage for the retailer to compete with the manufacturer. Under service competition, the retailer should strive to provide better services regardless of the market structure. Third, the manufacturer should pay 
attention to the service for standard products but reduce the service expenditures for customized products when his service cost coefficient is high. In addition, the manufacturer is encouraged to construct his customization production line and improve his customization level to provide more diversified products when customers are more sensitive to product customization. Finally, the manufacturer Stackelberg model is more profitable than the other two models for the overall SC, while the profit gap between the manufacturer and the retailer is quite large. Therefore, the coordination of the MS model is critical.

There are certain limitations in this paper that can be extended in future research. First, the demand is assumed to be linear and deterministic to simplify the model. In fact, the actual demand of the market is complex and stochastic, and considerable useful information can be obtained by analyzing the random demand. Second, the manufacturer and the retailer are exposed to the same information. Future research can consider an asymmetric information game model in a dual-channel SC with product customization. Finally, future research should consider more detailed modeling of customized products and refine customized features.

\section{Appendix}

Proof of Proposition 1. Substitute equation (3) into equation (1) and take the second-order partial differentiate $\Pi_{m}$ with respect to $w, p_{m}$, and $p_{z}$ to obtain the Hessian matrix:

$$
H_{M}=\left(\begin{array}{ccc}
-\left(\delta_{s}+2 \gamma_{c}+\gamma_{p}\right) & \gamma_{c} & \gamma_{c}+\gamma_{p} \\
\gamma_{c} & -2\left(\delta_{s}+\gamma_{c}+\gamma_{p}\right)+\frac{\gamma_{c}^{2}}{\delta_{s}+2 \gamma_{c}+\gamma_{p}} & 2 \gamma_{p}+\frac{\gamma_{c}\left(\gamma_{c}+\gamma_{p}\right)}{\delta_{s}+2 \gamma_{c}+\gamma_{p}} \\
\gamma_{c}+\gamma_{p} & 2 \gamma_{p}+\frac{\gamma_{c}\left(\gamma_{c}+\gamma_{p}\right)}{\delta_{s}+2 \gamma_{c}+\gamma_{p}} & -2\left(\delta_{z}+\gamma_{c}+2 \gamma_{p}\right)+\frac{\left(\gamma_{c}+\gamma_{p}\right)^{2}}{\delta_{s}+2 \gamma_{c}+\gamma_{p}}
\end{array}\right) .
$$

Clearly, $\left|H_{1}\right|=-\left(\delta_{s}+2 \gamma_{c}+\gamma_{p}\right)<0$ and $\left|H_{2}\right|=2\left(\delta_{s}+\right.$ $\left.2 \gamma_{c}+\gamma_{p}\right)\left(\delta_{s}+\gamma_{c}+\gamma_{p}\right)-\gamma_{c}^{2}>0$. Additionally, $\left|H_{3}\right|<0$ when

$$
\begin{aligned}
\gamma_{c} \gamma_{p}< & \frac{\left[2\left(\delta_{s}+2 \gamma_{c}+\gamma_{p}\right)\left(\delta_{s}+\gamma_{c}+\gamma_{p}\right)-\gamma_{c}^{2}\right]\left[2\left(\delta_{z}+\gamma_{c}+2 \gamma_{p}\right)-\left(\left(\gamma_{c}+\gamma_{p}\right)^{2} / \delta_{s}+2 \gamma_{c}+\gamma_{p}\right)\right]}{4\left(\gamma_{c}+\gamma_{p}\right)}-\frac{\left(\delta_{s}+\gamma_{c}+\gamma_{p}\right)\left(\gamma_{c}+\gamma_{p}\right)}{2} \\
& -\frac{\left(\delta_{z}+\gamma_{c}+2 \gamma_{p}\right) \gamma_{c}^{2}}{2\left(\gamma_{c}+\gamma_{p}\right)}-\frac{\left(\delta_{s}+2 \gamma_{c}+\gamma_{p}\right)\left[2 \gamma_{p}+\left(\gamma_{c}\left(\gamma_{c}+\gamma_{p}\right) / \delta_{s}+2 \gamma_{c}+\gamma_{p}\right)\right]^{2}}{4\left(\gamma_{c}+\gamma_{p}\right)},
\end{aligned}
$$

where $\quad A_{1}=\delta_{s}+\gamma_{c}+\gamma_{p}, \quad A_{2}=\delta_{z}+\gamma_{c}+2 \gamma_{p}, \quad A_{3}=\beta_{s}+$ $\theta_{c}+\theta_{p}, A_{4}=\beta_{z}+\theta_{c}+2 \theta_{p}$,

$$
\begin{aligned}
& A_{5}=4\left[\gamma_{p}+\frac{\gamma_{c}\left(\gamma_{c}+\gamma_{p}\right)}{2\left(A_{1}+\gamma_{c}\right)}\right]^{2}-4\left[\frac{\left(\gamma_{c}+\gamma_{p}\right)^{2}}{2\left(A_{1}+\gamma_{c}\right)}-A_{2}\right]\left[\frac{\gamma_{c}^{2}}{2\left(A_{1}+\gamma_{c}\right)}-A_{1}\right], \\
& A_{6}=4 \gamma_{c} \gamma_{p}\left(\gamma_{c}+\gamma_{p}\right)+2 A_{1}\left(\gamma_{c}+\gamma_{p}\right)^{2}+2 A_{2} \gamma_{c}^{2}+A_{5}\left(A_{1}+\gamma_{c}\right), \\
& B_{1}=\frac{1}{2} A_{5}\left[\alpha_{r}+c_{s} A_{1}-c_{z}\left(\gamma_{c}+\gamma_{p}\right)\right]-2\left[\gamma_{c} A_{2}+\gamma_{p}\left(\gamma_{c}+\gamma_{p}\right)\right]\left\{\alpha_{m}+\frac{\alpha_{r} \gamma_{c}}{2\left(A_{1}+\gamma_{c}\right)}\right.
\end{aligned}
$$


18

Complexity

$$
\begin{aligned}
& \left.+c_{s}\left(A_{1}-\frac{1}{2} \gamma_{c}\right)-\frac{c_{s} \gamma_{c}^{2}}{2\left(A_{1}+\gamma_{c}\right)}-c_{z}\left[\gamma_{p}+\frac{\gamma_{c}\left(\gamma_{c}+\gamma_{p}\right)}{2\left(A_{1}+\gamma_{c}\right)}\right]\right\}-2\left[A_{1}\left(\gamma_{c}+\gamma_{p}\right)+\gamma_{c} \gamma_{p}\right]\left\{\alpha_{z}+\frac{\alpha_{r}\left(\gamma_{c}+\gamma_{p}\right)}{2\left(A_{1}+\gamma_{c}\right)}\right. \\
& \left.-\frac{1}{2} c_{s}\left(\gamma_{c}+\gamma_{p}\right)-c_{s}\left[\gamma_{p}+\frac{\gamma_{c}\left(\gamma_{c}+\gamma_{p}\right)}{2\left(A_{1}+\gamma_{c}\right)}\right]-c_{z}\left[\frac{\left(\gamma_{c}+\gamma_{p}\right)^{2}}{2\left(A_{1}+\gamma_{c}\right)}-A_{2}\right]\right\} \text {, } \\
& D_{1}=\frac{1}{2}\left(A_{3}+\theta_{c}\right) A_{5}-2\left[\gamma_{c} A_{2}+\gamma_{p}\left(\gamma_{c}+\gamma_{p}\right)\right] \\
& \cdot\left[\frac{\gamma_{c}\left(A_{3}+\theta_{c}\right)}{2\left(A_{1}+\gamma_{c}\right)}-\theta_{c}\right]-2\left[A_{1}\left(\gamma_{c}+\gamma_{p}\right)+\gamma_{c} \gamma_{p}\right]\left[\frac{\left(\gamma_{c}+\gamma_{p}\right)\left(A_{3}+\theta_{c}\right)}{2\left(A_{1}+\gamma_{c}\right)}-\theta_{c}-\theta_{p}\right], \\
& G_{1}=-\frac{1}{2} \varphi_{2} A_{5}+2\left[\gamma_{c} A_{2}+\gamma_{p}\left(\gamma_{c}+\gamma_{p}\right)\right]\left[\varphi_{2}+\frac{\gamma_{c} \varphi_{2}}{2\left(A_{1}+\gamma_{c}\right)}\right]-2\left[A_{1}\left(\gamma_{c}+\gamma_{p}\right)+\gamma_{c} \gamma_{p}\right]\left[\varphi_{1}-\frac{\varphi_{2}\left(\gamma_{c}+\gamma_{p}\right)}{2\left(A_{1}+\gamma_{c}\right)}\right] \text {, } \\
& E_{1}=-\frac{1}{2} \theta_{c} A_{5}-2\left[\gamma_{c} A_{2}+\gamma_{p}\left(\gamma_{c}+\gamma_{p}\right)\right]\left[A_{3}-\frac{\gamma_{c} \theta_{c}}{2\left(A_{1}+\gamma_{c}\right)}\right]+2\left[A_{1}\left(\gamma_{c}+\gamma_{p}\right)+\gamma_{c} \gamma_{p}\right]\left[\theta_{p}+\frac{\theta_{c}\left(\gamma_{c}+\gamma_{p}\right)}{2\left(A_{1}+\gamma_{c}\right)}\right] \text {, } \\
& F_{1}=-\frac{1}{2}\left(\theta_{c}+\theta_{p}\right) A_{5}+2\left[\gamma_{c} A_{2}+\gamma_{p}\left(\gamma_{c}+\gamma_{p}\right)\right]\left[\theta_{p}+\frac{\gamma_{c}\left(\theta_{c}+\theta_{p}\right)}{2\left(A_{1}+\gamma_{c}\right)}\right] \\
& -2\left[A_{1}\left(\gamma_{c}+\gamma_{p}\right)+\gamma_{c} \gamma_{p}\right]\left[A_{4}-\frac{\left(\theta_{c}+\theta_{p}\right)\left(\gamma_{c}+\gamma_{p}\right)}{2\left(A_{1}+\gamma_{c}\right)}\right], \\
& B_{2}=-\left[\gamma_{c} A_{2}+\gamma_{p}\left(\gamma_{c}+\gamma_{p}\right)\right]\left[\alpha_{r}+c_{s} A_{1}-c_{z}\left(\gamma_{c}+\gamma_{p}\right)\right]+2\left[\left(\gamma_{c}+\gamma_{p}\right)^{2}-\left(A_{1}+\gamma_{c}\right) A_{2}\right]\left\{\alpha_{m}\right. \\
& \left.+\frac{\alpha_{r} \gamma_{c}}{2\left(A_{1}+\gamma_{c}\right)}+c_{s}\left(A_{1}-\frac{1}{2} \gamma_{c}\right)-\frac{c_{s} \gamma_{c}^{2}}{2\left(A_{1}+\gamma_{c}\right)}-c_{z}\left[\gamma_{p}+\frac{\gamma_{c}\left(\gamma_{c}+\gamma_{p}\right)}{2\left(A_{1}+\gamma_{c}\right)}\right]\right\}-2\left[\gamma_{c}\left(\gamma_{c}+\gamma_{p}\right)+\gamma_{p}\left(A_{1}+\gamma_{c}\right)\right]\left\{\alpha_{z}\right. \\
& \left.+\frac{\alpha_{r}\left(\gamma_{c}+\gamma_{p}\right)}{2\left(A_{1}+\gamma_{c}\right)}-\frac{1}{2} c_{s}\left(\gamma_{c}+\gamma_{p}\right)-c_{s}\left[\gamma_{p}+\frac{\gamma_{c}\left(\gamma_{c}+\gamma_{p}\right)}{2\left(A_{1}+\gamma_{c}\right)}\right]-c_{z}\left[\frac{\left(\gamma_{c}+\gamma_{p}\right)^{2}}{2\left(A_{1}+\gamma_{c}\right)}-A_{2}\right]\right\} \text {, } \\
& D_{2}=-\left(A_{3}+\theta_{c}\right)\left[\gamma_{c} A_{2}+\gamma_{p}\left(\gamma_{c}+\gamma_{p}\right)\right]+2\left[\left(\gamma_{c}+\gamma_{p}\right)^{2}-\left(A_{1}+\gamma_{c}\right) A_{2}\right]\left[\frac{\gamma_{c}\left(A_{3}+\theta_{c}\right)}{2\left(A_{1}+\gamma_{c}\right)}-\theta_{c}\right] \\
& -2\left[\gamma_{c}\left(\gamma_{c}+\gamma_{p}\right)+\gamma_{p}\left(A_{1}+\gamma_{c}\right)\right]\left[\frac{\left(\gamma_{c}+\gamma_{p}\right)\left(A_{3}+\theta_{c}\right)}{2\left(A_{1}+\gamma_{c}\right)}-\theta_{c}-\theta_{p}\right], \\
& E_{2}=\theta_{c}\left[\gamma_{c} A_{2}+\gamma_{p}\left(\gamma_{c}+\gamma_{p}\right)\right]+2\left[\left(\gamma_{c}+\gamma_{p}\right)^{2}-\left(A_{1}+\gamma_{c}\right) A_{2}\right]\left[A_{3}-\frac{\gamma_{c} \theta_{c}}{2\left(A_{1}+\gamma_{c}\right)}\right] \\
& +2\left[\gamma_{c}\left(\gamma_{c}+\gamma_{p}\right)+\gamma_{p}\left(A_{1}+\gamma_{c}\right)\right]\left[\theta_{p}+\frac{\theta_{c}\left(\gamma_{c}+\gamma_{p}\right)}{2\left(A_{1}+\gamma_{c}\right)}\right], \\
& F_{2}=\left(\theta_{c}+\theta_{p}\right)\left[\gamma_{c} A_{2}+\gamma_{p}\left(\gamma_{c}+\gamma_{p}\right)\right]-2\left[\left(\gamma_{c}+\gamma_{p}\right)^{2}-\left(A_{1}+\gamma_{c}\right) A_{2}\right]\left[\theta_{p}+\frac{\gamma_{c}\left(\theta_{c}+\theta_{p}\right)}{2\left(A_{1}+\gamma_{c}\right)}\right] \\
& -2\left[\gamma_{c}\left(\gamma_{c}+\gamma_{p}\right)+\gamma_{p}\left(A_{1}+\gamma_{c}\right)\right]\left[A_{4}-\frac{\left(\theta_{c}+\theta_{p}\right)\left(\gamma_{c}+\gamma_{p}\right)}{2\left(A_{1}+\gamma_{c}\right)}\right], \\
& G_{2}=\varphi_{2}\left[\gamma_{c} A_{2}+\gamma_{p}\left(\gamma_{c}+\gamma_{p}\right)\right]-2\left[\left(\gamma_{c}+\gamma_{p}\right)^{2}-\left(A_{1}+\gamma_{c}\right) A_{2}\right]\left[\varphi_{2}+\frac{\gamma_{c} \varphi_{2}}{2\left(A_{1}+\gamma_{c}\right)}\right]
\end{aligned}
$$


Complexity

19

$$
\begin{aligned}
& -2\left[\gamma_{c}\left(\gamma_{c}+\gamma_{p}\right)+\gamma_{p}\left(A_{1}+\gamma_{c}\right)\right]\left[\varphi_{1}-\frac{\varphi_{2}\left(\gamma_{c}+\gamma_{p}\right)}{2\left(A_{1}+\gamma_{c}\right)}\right] \\
& B_{3}=-\left[A_{1}\left(\gamma_{c}+\gamma_{p}\right)+\gamma_{c} \gamma_{p}\right]\left[\alpha_{r}+c_{s} A_{1}-c_{z}\left(\gamma_{c}+\gamma_{p}\right)\right]-2\left[\gamma_{c}\left(\gamma_{c}+\gamma_{p}\right)+\gamma_{p}\left(A_{1}+\gamma_{c}\right)\right]\left\{\alpha_{m}\right. \\
& \left.+\frac{\alpha_{r} \gamma_{c}}{2\left(A_{1}+\gamma_{c}\right)}+c_{s}\left(A_{1}-\frac{1}{2} \gamma_{c}\right)-\frac{c_{s} \gamma_{c}^{2}}{2\left(A_{1}+\gamma_{c}\right)}-c_{z}\left[\gamma_{p}+\frac{\gamma_{c}\left(\gamma_{c}+\gamma_{p}\right)}{2\left(A_{1}+\gamma_{c}\right)}\right]\right\} \\
& +2\left[\gamma_{c}^{2}-A_{1}\left(A_{1}+\gamma_{c}\right)\right]\left\{\alpha_{z}+\frac{\alpha_{r}\left(\gamma_{c}+\gamma_{p}\right)}{2\left(A_{1}+\gamma_{c}\right)}-\frac{1}{2} c_{s}\left(\gamma_{c}+\gamma_{p}\right)-c_{s}\left[\gamma_{p}+\frac{\gamma_{c}\left(\gamma_{c}+\gamma_{p}\right)}{2\left(A_{1}+\gamma_{c}\right)}\right]-c_{z}\left[\frac{\left(\gamma_{c}+\gamma_{p}\right)^{2}}{2\left(A_{1}+\gamma_{c}\right)}-A_{2}\right]\right\}, \\
& D_{3}=-\left(A_{3}+\theta_{c}\right)\left[A_{1}\left(\gamma_{c}+\gamma_{p}\right)+\gamma_{c} \gamma_{p}\right]-2\left[\gamma_{c}\left(\gamma_{c}+\gamma_{p}\right)+\gamma_{p}\left(A_{1}+\gamma_{c}\right)\right]\left[\frac{\gamma_{c}\left(A_{3}+\theta_{c}\right)}{2\left(A_{1}+\gamma_{c}\right)}-\theta_{c}\right] \\
& +2\left[\gamma_{c}^{2}-A_{1}\left(A_{1}+\gamma_{c}\right)\right]\left[\frac{\left(\gamma_{c}+\gamma_{p}\right)\left(A_{3}+\theta_{c}\right)}{2\left(A_{1}+\gamma_{c}\right)}-\theta_{c}-\theta_{p}\right] \\
& E_{3}=\theta_{c}\left[A_{1}\left(\gamma_{c}+\gamma_{p}\right)+\gamma_{c} \gamma_{p}\right]-2\left[\gamma_{c}\left(\gamma_{c}+\gamma_{p}\right)+\gamma_{p}\left(A_{1}+\gamma_{c}\right)\right]\left[A_{3}-\frac{\gamma_{c} \theta_{c}}{2\left(A_{1}+\gamma_{c}\right)}\right] \\
& -2\left[\gamma_{c}^{2}-A_{1}\left(A_{1}+\gamma_{c}\right)\right]\left[\theta_{p}+\frac{\theta_{c}\left(\gamma_{c}+\gamma_{p}\right)}{2\left(A_{1}+\gamma_{c}\right)}\right] \\
& F_{3}=\left(\theta_{c}+\theta_{p}\right)\left[A_{1}\left(\gamma_{c}+\gamma_{p}\right)+\gamma_{c} \gamma_{p}\right]+2\left[\gamma_{c}\left(\gamma_{c}+\gamma_{p}\right)+\gamma_{p}\left(A_{1}+\gamma_{c}\right)\right]\left[\theta_{p}+\frac{\gamma_{c}\left(\theta_{c}+\theta_{p}\right)}{2\left(A_{1}+\gamma_{c}\right)}\right] \\
& +2\left[\gamma_{c}^{2}-A_{1}\left(A_{1}+\gamma_{c}\right)\right]\left[A_{4}-\frac{\left(\theta_{c}+\theta_{p}\right)\left(\gamma_{c}+\gamma_{p}\right)}{2\left(A_{1}+\gamma_{c}\right)}\right] \\
& G_{3}=\varphi_{2}\left[A_{1}\left(\gamma_{c}+\gamma_{p}\right)+\gamma_{c} \gamma_{p}\right]+2\left[\gamma_{c}\left(\gamma_{c}+\gamma_{p}\right)+\gamma_{p}\left(A_{1}+\gamma_{c}\right)\right]\left[\varphi_{2}+\frac{\gamma_{c} \varphi_{2}}{2\left(A_{1}+\gamma_{c}\right)}\right] \\
& +2\left[\gamma_{c}^{2}-A_{1}\left(A_{1}+\gamma_{c}\right)\right]\left[\varphi_{1}-\frac{\varphi_{2}\left(\gamma_{c}+\gamma_{p}\right)}{2\left(A_{1}+\gamma_{c}\right)}\right] \\
& B_{4}=\frac{\alpha_{r} A_{6}}{2\left(A_{1}+\gamma_{c}\right)}+\frac{\gamma_{c} B_{2}+\left(\gamma_{c}+\gamma_{p}\right) B_{3}+\left(A_{1}+\gamma_{c}\right) B_{1}}{2\left(A_{1}+\gamma_{c}\right)}, \\
& D_{4}=\frac{A_{6}\left(A_{3}+\theta_{c}\right)}{2\left(A_{1}+\gamma_{c}\right)}+\frac{\gamma_{c} D_{2}+\left(\gamma_{c}+\gamma_{p}\right) D_{3}+\left(A_{1}+\gamma_{c}\right) D_{1}}{2\left(A_{1}+\gamma_{c}\right)} \\
& E_{4}=-\frac{\theta_{c} A_{6}}{2\left(A_{1}+\gamma_{c}\right)}+\frac{\gamma_{c} E_{2}+\left(\gamma_{c}+\gamma_{p}\right) E_{3}+\left(A_{1}+\gamma_{c}\right) E_{1}}{2\left(A_{1}+\gamma_{c}\right)}, \\
& F_{4}=-\frac{A_{6}\left(\theta_{c}+\theta_{p}\right)}{2\left(A_{1}+\gamma_{c}\right)}+\frac{\gamma_{c} F_{2}+\left(\gamma_{c}+\gamma_{p}\right) F_{3}+\left(A_{1}+\gamma_{c}\right) F_{1}}{2\left(A_{1}+\gamma_{c}\right)}, \\
& G_{4}=-\frac{\varphi_{2} A_{6}}{2\left(A_{1}+\gamma_{c}\right)}+\frac{\gamma_{c} G_{2}+\left(\gamma_{c}+\gamma_{p}\right) G_{3}+\left(A_{1}+\gamma_{c}\right) G_{1}}{2\left(A_{1}+\gamma_{c}\right)} \text {. }
\end{aligned}
$$


Therefore, the profit function of the manufacturer is jointly concave in $w, p_{m}$, and $p_{z}$ under the given service levels provided that

$$
\begin{aligned}
\gamma_{c} \gamma_{p}< & \frac{\left[2\left(\delta_{s}+2 \gamma_{c}+\gamma_{p}\right)\left(\delta_{s}+\gamma_{c}+\gamma_{p}\right)-\gamma_{c}^{2}\right]\left[2\left(\delta_{z}+\gamma_{c}+2 \gamma_{p}\right)-\left(\left(\gamma_{c}+\gamma_{p}\right)^{2} / \delta_{s}+2 \gamma_{c}+\gamma_{p}\right)\right]}{4\left(\gamma_{c}+\gamma_{p}\right)}-\frac{\left(\delta_{s}+\gamma_{c}+\gamma_{p}\right)\left(\gamma_{c}+\gamma_{p}\right)}{2} \\
& -\frac{\left(\delta_{z}+\gamma_{c}+2 \gamma_{p}\right) \gamma_{c}^{2}}{2\left(\gamma_{c}+\gamma_{p}\right)}-\frac{\left(\delta_{s}+2 \gamma_{c}+\gamma_{p}\right)\left[2 \gamma_{p}+\left(\gamma_{c}\left(\gamma_{c}+\gamma_{p}\right) / \delta_{s}+2 \gamma_{c}+\gamma_{p}\right)\right]^{2}}{4\left(\gamma_{c}+\gamma_{p}\right)} .
\end{aligned}
$$

Proof of Proposition 2. For the concavity of $\Pi_{r},\left(\partial^{2} \Pi_{r} / \partial s_{r}^{2}\right)=$ $-\mathrm{H}_{5}$ should be negative. Therefore, the profit function of the retailer is concave in $s_{r}$ provided that $\eta_{2}>\left(2\left(D_{4}-\right.\right.$
$\left.\left.D_{1}\right) / A_{6}\right)\left[A_{3}+\theta_{c}+\left(\gamma_{c} D_{2}+\left(\gamma_{c}+\gamma_{p}\right) D_{3}-\left(A_{1}+\gamma_{c}\right) D_{4} / A_{6}\right)\right]$, where

$$
\begin{aligned}
& H_{1}=\frac{D_{4}-D_{1}}{A_{6}}\left[\alpha_{r}+\frac{\gamma_{c} B_{2}+\left(\gamma_{c}+\gamma_{p}\right) B_{3}-\left(A_{1}+\gamma_{c}\right) B_{4}}{A_{6}}\right]+\frac{B_{4}-B_{1}}{A_{6}}\left[A_{3}+\theta_{c}+\frac{\gamma_{c} D_{2}+\left(\gamma_{c}+\gamma_{p}\right) D_{3}-\left(A_{1}+\gamma_{c}\right) D_{4}}{A_{6}}\right], \\
& H_{2}=\frac{D_{4}-D_{1}}{A_{6}}\left[\frac{\gamma_{c} E_{2}+\left(\gamma_{c}+\gamma_{p}\right) E_{3}-\left(A_{1}+\gamma_{c}\right) E_{4}}{A_{6}}-\theta_{c}\right]+\frac{E_{4}-E_{1}}{A_{6}}\left[A_{3}+\theta_{c}+\frac{\gamma_{c} D_{2}+\left(\gamma_{c}+\gamma_{p}\right) D_{3}-\left(A_{1}+\gamma_{c}\right) D_{4}}{A_{6}}\right], \\
& H_{3}=\frac{D_{4}-D_{1}}{A_{6}}\left[\frac{\gamma_{c} F_{2}+\left(\gamma_{c}+\gamma_{p}\right) F_{3}-\left(A_{1}+\gamma_{c}\right) F_{4}}{A_{6}}-\theta_{c}-\theta_{p}\right]+\frac{F_{4}-F_{1}}{A_{6}}\left[A_{3}+\theta_{c}+\frac{\gamma_{c} D_{2}+\left(\gamma_{c}+\gamma_{p}\right) D_{3}-\left(A_{1}+\gamma_{c}\right) D_{4}}{A_{6}}\right], \\
& H_{4}=\frac{D_{4}-D_{1}}{A_{6}}\left[\frac{\gamma_{c} G_{2}+\left(\gamma_{c}+\gamma_{p}\right) G_{3}-\left(A_{1}+\gamma_{c}\right) G_{4}}{A_{6}}-\varphi_{2}\right]+\frac{G_{4}-G_{1}}{A_{6}}\left[A_{3}+\theta_{c}+\frac{\gamma_{c} D_{2}+\left(\gamma_{c}+\gamma_{p}\right) D_{3}-\left(A_{1}+\gamma_{c}\right) D_{4}}{A_{6}}\right], \\
& H_{5}=\eta_{2}-\frac{2\left(D_{4}-D_{1}\right)}{A_{6}}\left[A_{3}+\theta_{c}+\frac{\gamma_{c} D_{2}+\left(\gamma_{c}+\gamma_{p}\right) D_{3}-\left(A_{1}+\gamma_{c}\right) D_{4}}{A_{6}}\right] .
\end{aligned}
$$

Proof of Proposition 3. Take the second-order partial derivatives of $\Pi_{m}$ with respect to $s_{m}, s_{z}$, and $\mu$ and obtain the Hessian matrix:

$$
H_{M}=\left(\begin{array}{ccc}
I_{2} & I_{3} & I_{4} \\
J_{2} & J_{3} & J_{4} \\
K_{2} & K_{3} & K_{4}
\end{array}\right) .
$$

$\left|H_{1}\right|=I_{2}, \quad\left|H_{2}\right|=I_{2} J_{3}-I_{3} J_{2}, \quad$ and $\quad\left|H_{3}\right|=I_{2}\left(J_{3} K_{4}-\right.$ $\left.J_{4} K_{3}\right)+J_{2}\left(I_{4} K_{3}-I_{3} K_{4}\right)+K_{2}\left(I_{3} J_{4}-I_{4} J_{3}\right)$. To ensure that $\Pi_{m}$ is jointly concave in $s_{m}, s_{z}$, and $\mu$, the parameters should meet the conditions that $I_{2}<0, I_{2} J_{3}-I_{3} J_{2}>0$, and $I_{2}\left(J_{3} K_{4}-J_{4} K_{3}\right)+J_{2}\left(I_{4} K_{3}-I_{3} K_{4}\right)+K_{2}\left(I_{3} J_{4}-I_{4} J_{3}\right)<0$, where

$$
\begin{aligned}
& D_{5}=\frac{D_{1} H_{1}+B_{1} H_{5}}{A_{6} H_{5}}-c_{s}, \\
& D_{6}=\frac{D_{1} H_{2}+E_{1} H_{5}}{A_{6} H_{5}},
\end{aligned}
$$




$$
\begin{aligned}
& D_{7}=\frac{D_{1} H_{3}+F_{1} H_{5}}{A_{6} H_{5}}, \\
& D_{8}=\frac{D_{1} H_{4}+G_{1} H_{5}}{A_{6} H_{5}}, \\
& D_{9}=\alpha_{r}-\left(A_{1}+\gamma_{c}\right) \frac{B_{4} H_{5}+D_{4} H_{1}}{A_{6} H_{5}}+\gamma_{c} \frac{B_{2} H_{5}+D_{2} H_{1}}{A_{6} H_{5}}+\left(\gamma_{c}+\gamma_{p}\right) \frac{B_{3} H_{5}+D_{3} H_{1}}{A_{6} H_{5}}+\frac{H_{1}\left(A_{3}+\theta_{c}\right)}{H_{5}}, \\
& D_{10}=-\left(A_{1}+\gamma_{c}\right) \frac{E_{4} H_{5}+D_{4} H_{2}}{A_{6} H_{5}}+\gamma_{c} \frac{E_{2} H_{5}+D_{2} H_{2}}{A_{6} H_{5}}+\left(\gamma_{c}+\gamma_{p}\right) \frac{E_{3} H_{5}+D_{3} H_{2}}{A_{6} H_{5}}+\frac{H_{2}\left(A_{3}+\theta_{c}\right)}{H_{5}}-\theta_{c} \text {, } \\
& D_{11}=-\left(A_{1}+\gamma_{c}\right) \frac{F_{4} H_{5}+D_{4} H_{3}}{A_{6} H_{5}}+\gamma_{c} \frac{F_{2} H_{5}+D_{2} H_{3}}{A_{6} H_{5}}+\left(\gamma_{c}+\gamma_{p}\right) \frac{F_{3} H_{5}+D_{3} H_{3}}{A_{6} H_{5}}+\frac{H_{3}\left(A_{3}+\theta_{c}\right)}{H_{5}}-\theta_{c}-\theta_{p} \text {, } \\
& D_{12}=-\left(A_{1}+\gamma_{c}\right) \frac{G_{4} H_{5}+D_{4} H_{4}}{A_{6} H_{5}}+\gamma_{c} \frac{G_{2} H_{5}+D_{2} H_{4}}{A_{6} H_{5}}+\left(\gamma_{c}+\gamma_{p}\right) \frac{G_{3} H_{5}+D_{3} H_{4}}{A_{6} H_{5}}+\frac{H_{4}\left(A_{3}+\theta_{c}\right)}{H_{5}}-\varphi_{2} \text {, } \\
& E_{5}=\frac{D_{2} H_{1}+B_{2} H_{5}}{A_{6} H_{5}}-c_{s} \text {, } \\
& E_{6}=\frac{D_{2} H_{2}+E_{2} H_{5}}{A_{6} H_{5}}, \\
& E_{7}=\frac{D_{2} H_{3}+F_{2} H_{5}}{A_{6} H_{5}}, \\
& E_{8}=\frac{D_{2} H_{4}+G_{2} H_{5}}{A_{6} H_{5}}, \\
& E_{9}=\alpha_{m}+\gamma_{c} \frac{B_{4} H_{5}+D_{4} H_{1}}{A_{6} H_{5}}-A_{1} \frac{B_{2} H_{5}+D_{2} H_{1}}{A_{6} H_{5}}+\gamma_{p} \frac{B_{3} H_{5}+D_{3} H_{1}}{A_{6} H_{5}}-\frac{H_{1} \theta_{c}}{H_{5}}, \\
& E_{10}=\gamma_{c} \frac{E_{4} H_{5}+D_{4} H_{2}}{A_{6} H_{5}}-A_{1} \frac{E_{2} H_{5}+D_{2} H_{2}}{A_{6} H_{5}}+\gamma_{p} \frac{E_{3} H_{5}+D_{3} H_{2}}{A_{6} H_{5}}-\frac{H_{2} \theta_{c}}{H_{5}}+A_{3} \text {, } \\
& E_{11}=\gamma_{c} \frac{F_{4} H_{5}+D_{4} H_{3}}{A_{6} H_{5}}-A_{1} \frac{F_{2} H_{5}+D_{2} H_{3}}{A_{6} H_{5}}+\gamma_{p} \frac{F_{3} H_{5}+D_{3} H_{3}}{A_{6} H_{5}}-\frac{H_{3} \theta_{c}}{H_{5}}-\theta_{p} \text {, } \\
& E_{12}=\gamma_{c} \frac{G_{4} H_{5}+D_{4} H_{4}}{A_{6} H_{5}}-A_{1} \frac{G_{2} H_{5}+D_{2} H_{4}}{A_{6} H_{5}}+\gamma_{p} \frac{G_{3} H_{5}+D_{3} H_{4}}{A_{6} H_{5}}-\frac{H_{4} \theta_{c}}{H_{5}}-\varphi_{2} \text {, } \\
& F_{5}=\frac{D_{3} H_{1}+B_{3} H_{5}}{A_{6} H_{5}}-c_{z} \text {, } \\
& F_{6}=\frac{D_{3} H_{2}+E_{3} H_{5}}{A_{6} H_{5}}, \\
& F_{7}=\frac{D_{3} H_{3}+F_{3} H_{5}}{A_{6} H_{5}}, \\
& F_{8}=\frac{D_{3} H_{4}+G_{3} H_{5}}{A_{6} H_{5}}, \\
& F_{9}=\alpha_{z}+\left(\gamma_{c}+\gamma_{p}\right) \frac{B_{4} H_{5}+D_{4} H_{1}}{A_{6} H_{5}}+\gamma_{p} \frac{B_{2} H_{5}+D_{2} H_{1}}{A_{6} H_{5}}-A_{2} \frac{B_{3} H_{5}+D_{3} H_{1}}{A_{6} H_{5}}-\frac{H_{1}\left(\theta_{c}+\theta_{p}\right)}{H_{5}}, \\
& F_{10}=\left(\gamma_{c}+\gamma_{p}\right) \frac{E_{4} H_{5}+D_{4} H_{2}}{A_{6} H_{5}}+\gamma_{p} \frac{E_{2} H_{5}+D_{2} H_{2}}{A_{6} H_{5}}-A_{2} \frac{E_{3} H_{5}+D_{3} H_{2}}{A_{6} H_{5}}-\frac{H_{2}\left(\theta_{c}+\theta_{p}\right)}{H_{5}}-\theta_{p},
\end{aligned}
$$




$$
\begin{aligned}
F_{11} & =\left(\gamma_{c}+\gamma_{p}\right) \frac{F_{4} H_{5}+D_{4} H_{3}}{A_{6} H_{5}}+\gamma_{p} \frac{F_{2} H_{5}+D_{2} H_{3}}{A_{6} H_{5}}-A_{2} \frac{F_{3} H_{5}+D_{3} H_{3}}{A_{6} H_{5}}-\frac{H_{3}\left(\theta_{c}+\theta_{p}\right)}{H_{5}}+A_{4} \\
F_{12} & =\left(\gamma_{c}+\gamma_{p}\right) \frac{G_{4} H_{5}+D_{4} H_{4}}{A_{6} H_{5}}+\gamma_{p} \frac{G_{2} H_{5}+D_{2} H_{4}}{A_{6} H_{5}}-A_{2} \frac{G_{3} H_{5}+D_{3} H_{4}}{A_{6} H_{5}}-\frac{H_{4}\left(\theta_{c}+\theta_{p}\right)}{H_{5}}+\varphi_{1} \\
I_{1} & =D_{6} D_{9}+D_{5} D_{10}+E_{6} E_{9}+E_{5} E_{10}+F_{6} F_{9}+F_{5} F_{10} \\
I_{2} & =2 D_{6} D_{10}+2 E_{6} E_{10}+2 F_{6} F_{10}-\eta_{1} \\
I_{3} & =D_{6} D_{11}+D_{7} D_{10}+E_{6} E_{11}+E_{7} E_{10}+F_{6} F_{11}+F_{7} F_{10} \\
I_{4} & =D_{6} D_{12}+D_{8} D_{10}+E_{6} E_{12}+E_{8} E_{10}+F_{6} F_{12}+F_{8} F_{10} \\
J_{1} & =D_{7} D_{9}+D_{5} D_{11}+E_{7} E_{9}+E_{5} E_{11}+F_{7} F_{9}+F_{5} F_{11} \\
J_{2} & =D_{7} D_{10}+D_{6} D_{11}+E_{7} E_{10}+E_{6} E_{11}+F_{7} F_{10}+F_{6} F_{11} \\
J_{3} & =2 D_{7} D_{11}+2 E_{7} E_{11}+2 F_{7} F_{11}-\eta_{1}, \\
J_{4} & =D_{7} D_{12}+D_{8} D_{11}+E_{7} E_{12}+E_{8} E_{11}+F_{7} F_{12}+F_{8} F_{11} \\
K_{1} & =D_{8} D_{9}+D_{5} D_{12}+E_{8} E_{9}+E_{5} E_{12}+F_{8} F_{9}+F_{5} F_{12} \\
K_{2} & =D_{8} D_{10}+D_{6} D_{12}+E_{8} E_{10}+E_{6} E_{12}+F_{8} F_{10}+F_{6} F_{12} \\
K_{3} & =D_{8} D_{11}+D_{7} D_{12}+E_{8} E_{11}+E_{7} E_{12}+F_{8} F_{11}+F_{7} F_{12} \\
K_{4} & =2 D_{8} D_{12}+2 E_{8} E_{12}+2 F_{8} F_{12}-C .
\end{aligned}
$$

Then, $\Pi_{m}$ can be maximized jointly with respect to $s_{m}, s_{z}$, and $\mu$.

Proof of Proposition 4. Take the second-order partial differentiate $\Pi_{m}$ with respect to $p_{m}$ and $p_{z}$ to obtain the Hessian matrix:

$$
H_{M}=\left(\begin{array}{cc}
-2 A_{1} & 2 \gamma_{p} \\
2 \gamma_{p} & -2 A_{2}
\end{array}\right) .
$$

Clearly, $\left|H_{1}\right|=-2 A_{1}<0$ and $\left|H_{2}\right|=4 A_{1} A_{2}-4 \gamma_{p}^{2}>0$. Therefore, $\Pi_{m}$ is jointly concave in $p_{m}$ and $p_{z}$. Let $\left(\partial \Pi_{m} / \partial p_{m}\right)=0$ and $\left(\partial \Pi_{m} / \partial p_{z}\right)=0$ to derive the optimal $p_{m}$ and $p_{z}$.

Proof of Proposition 5. The second-order partial differentiate $\Pi_{r}$ with respect to $p_{r}$ should be negative to ensure the concavity of $\Pi_{r}$. Since $\left(\partial^{2} \Pi_{r} / \partial p_{r}^{2}\right)=\left(6 \gamma_{c} \gamma_{p} \quad\left(\gamma_{c}+\gamma_{p}\right)+\right.$ $\left.3 \gamma_{c}^{2} A_{2}+3\left(\gamma_{c}+\gamma_{p}\right)^{2} A_{1} / 4\left(A_{1} A_{2}-\gamma_{p}^{2}\right)\right)-A_{1}-\gamma_{c}, \gamma_{c}$ should be larger than $\left(6 \gamma_{c} \gamma_{p}\left(\gamma_{c}+\gamma_{p}\right)+3 \gamma_{c}^{2} A_{2}+3\left(\gamma_{c}+\gamma_{p}\right)^{2} A_{1} /\right.$ $\left.4\left(A_{1} A_{2}-\gamma_{p}^{2}\right)\right)-A_{1}$.

The unique optimal solution can be obtained by $p_{r}^{*}=(1 / 2) c_{s}-\left(L_{1}+L_{2} s_{r}+L_{3} s_{m}+L_{4} s_{z}+L_{5} \mu / 2 L_{6}\right)$, where

$$
\begin{aligned}
L_{1}= & \alpha_{r}+\frac{\gamma_{c}\left[2 A_{2} \alpha_{m}+2 \gamma_{p} \alpha_{z}-\gamma_{p} c_{s}\left(\gamma_{c}+3 \gamma_{p}\right)+2 A_{2} c_{s}\left(A_{1}-(1 / 2) \gamma_{c}\right)\right]}{4\left(A_{1} A_{2}-\gamma_{p}^{2}\right)} \\
& +\frac{\left(\gamma_{c}+\gamma_{p}\right)\left[2 \gamma_{p} \alpha_{m}+2 A_{1} \alpha_{z}-A_{1} c_{s}\left(\gamma_{c}+\gamma_{p}\right)-\gamma_{c} \gamma_{p} c_{s}+2 c_{z}\left(A_{1} A_{2}-\gamma_{p}^{2}\right)\right]}{4\left(A_{1} A_{2}-\gamma_{p}^{2}\right)}, \\
L_{2}= & A_{3}+\theta_{c}-\frac{\gamma_{c}\left[\gamma_{p}\left(\theta_{c}+\theta_{p}\right)+A_{2} \theta_{c}\right]+\left(\gamma_{c}+\gamma_{p}\right)\left[A_{1}\left(\theta_{c}+\theta_{p}\right)+\gamma_{p} \theta_{c}\right]}{2\left(A_{1} A_{2}-\gamma_{p}^{2}\right)},
\end{aligned}
$$


Complexity

23

$$
\begin{aligned}
& L_{3}=-\theta_{c}+\frac{\gamma_{c}\left(A_{2} A_{3}-\gamma_{p} \theta_{p}\right)+\left(\gamma_{c}+\gamma_{p}\right)\left(\gamma_{p} A_{3}-A_{1} \theta_{p}\right)}{2\left(A_{1} A_{2}-\gamma_{p}^{2}\right)}, \\
& L_{4}=-\left(\theta_{c}+\theta_{p}\right)+\frac{\gamma_{c}\left(\gamma_{p} A_{4}-A_{2} \theta_{p}\right)+\left(\gamma_{c}+\gamma_{p}\right)\left(A_{1} A_{4}-\gamma_{p} \theta_{p}\right)}{2\left(A_{1} A_{2}-\gamma_{p}^{2}\right)}, \\
& L_{5}=\frac{\gamma_{c}\left(\gamma_{p} \varphi_{1}-A_{2} \varphi_{2}\right)+\left(\gamma_{c}+\gamma_{p}\right)\left(A_{1} \varphi_{1}-\gamma_{p} \varphi_{2}\right)}{2\left(A_{1} A_{2}-\gamma_{p}^{2}\right)}-\varphi_{2}, \\
& L_{6}=-\left(A_{1}+\gamma_{c}\right)+\frac{3 \gamma_{c}\left[\gamma_{p}\left(\gamma_{c}+\gamma_{p}\right)+A_{2} \gamma_{c}\right]+3\left(\gamma_{c}+\gamma_{p}\right)\left[A_{1}\left(\gamma_{c}+\gamma_{p}\right)+\gamma_{c} \gamma_{p}\right]}{4\left(A_{1} A_{2}-\gamma_{p}^{2}\right)} .
\end{aligned}
$$

(A.9)

Proof of Proposition 6. or the concavity of $\Pi_{r},\left(\partial^{2} \Pi_{r} / \partial s_{r}^{2}\right)=$ $-\left(L_{2} M_{2} / 2 L_{6}\right)-\eta_{2}$ should be negative. Therefore, the profit function of the retailer is concave in $s_{r}$ provided that $\eta_{2}>-\left(L_{2} M_{2} / 2 L_{6}\right)$, where

$$
\begin{aligned}
M_{1}= & \alpha_{r}-\frac{1}{2}\left(A_{1}+\gamma_{c}\right)\left(c_{s}-\frac{L_{1}}{L_{6}}\right)+\frac{\gamma_{c}}{4\left(A_{1} A_{2}-\gamma_{p}^{2}\right)} \\
& \cdot\left\{2 A_{2} \alpha_{m}+2 \gamma_{p} \alpha_{z}-\gamma_{p} c_{s}\left(\gamma_{c}+3 \gamma_{p}\right)+2 A_{2} c_{s}\left(A_{1}-\frac{1}{2} \gamma_{c}\right)+\frac{3}{2}\left(c_{s}-\frac{L_{1}}{L_{6}}\right)\left[\gamma_{p}\left(\gamma_{c}+\gamma_{p}\right)+A_{2} \gamma_{c}\right]\right\}+\frac{\gamma_{c}+\gamma_{p}}{4\left(A_{1} A_{2}-\gamma_{p}^{2}\right)} \\
& \cdot\left\{2 \gamma_{p} \alpha_{m}+2 A_{1} \alpha_{z}-A_{1} c_{s}\left(\gamma_{c}+\gamma_{p}\right)-\gamma_{c} \gamma_{p} c_{s}+2 c_{z}\left(A_{1} A_{2}-\gamma_{p}^{2}\right)+\frac{3}{2}\left(c_{s}-\frac{L_{1}}{L_{6}}\right)\left[A_{1}\left(\gamma_{c}+\gamma_{p}\right)+\gamma_{c} \gamma_{p}\right]\right\}, \\
M_{2}= & \frac{L_{2}}{2 L_{6}}\left(A_{1}+\gamma_{c}\right)-\frac{\gamma_{c}}{4\left(A_{1} A_{2}-\gamma_{p}^{2}\right)}\left\{\frac{3 L_{2}}{2 L_{6}}\left[\gamma_{p}\left(\gamma_{c}+\gamma_{p}\right)+A_{2} \gamma_{c}\right]+2\left[\gamma_{p}\left(\theta_{c}+\theta_{p}\right)+A_{2} \theta_{c}\right]\right\} \\
& -\frac{\gamma_{c}+\gamma_{p}}{4\left(A_{1} A_{2}-\gamma_{p}^{2}\right)}\left\{\frac{3 L_{2}}{2 L_{6}}\left[A_{1}\left(\gamma_{c}+\gamma_{p}\right)+\gamma_{c} \gamma_{p}\right]+2\left[A_{1}\left(\theta_{c}+\theta_{p}\right)+\gamma_{p} \theta_{c}\right]\right\}+A_{3}+\theta_{c}, \\
M_{3}= & \frac{L_{3}}{2 L_{6}}\left(A_{1}+\gamma_{c}\right)-\frac{\gamma_{c}}{4\left(A_{1} A_{2}-\gamma_{p}^{2}\right)}\left\{\frac{3 L_{3}}{2 L_{6}}\left[\gamma_{p}\left(\gamma_{c}+\gamma_{p}\right)+A_{2} \gamma_{c}\right]+2\left(\gamma_{p} \theta_{p}-A_{2} A_{3}\right)\right\} \\
& -\frac{\gamma_{c}+\gamma_{p}}{4\left(A_{1} A_{2}-\gamma_{p}^{2}\right)}\left\{\frac{3 L_{3}}{2 L_{6}}\left[A_{1}\left(\gamma_{c}+\gamma_{p}\right)+\gamma_{c} \gamma_{p}\right]+2\left(\gamma_{p} A_{3}-A_{1} \theta_{p}\right)\right\}-\theta_{c}, \\
M_{4}= & \frac{L_{4}}{2 L_{6}}\left(A_{1}+\gamma_{c}\right)+\frac{\gamma_{c}}{4\left(A_{1} A_{2}-\gamma_{p}^{2}\right)}\left\{-\frac{3 L_{4}}{2 L_{6}}\left[\gamma_{p}\left(\gamma_{c}+\gamma_{p}\right)+A_{2} \gamma_{c}\right]+2\left(\gamma_{p} A_{4}-A_{2} \theta_{p}\right)\right\} \\
& +\frac{\gamma_{c}+\gamma_{p}}{4\left(A_{1} A_{2}-\gamma_{p}^{2}\right)}\left\{-\frac{3 L_{4}}{2 L_{6}}\left[A_{1}\left(\gamma_{c}+\gamma_{p}\right)+\gamma_{c} \gamma_{p}\right]+2\left(A_{1} A_{4}-\gamma_{p} \theta_{p}\right)\right\}-\theta_{c}-\theta_{p}, \\
M_{5}= & \frac{L_{5}}{2 L_{6}}\left(A_{1}+\gamma_{c}\right)+\frac{\gamma_{c}}{4\left(A_{1} A_{2}-\gamma_{p}^{2}\right)}\left\{-\frac{3 L_{5}}{2 L_{6}}\left[A_{1}\left(\gamma_{c}+\gamma_{p}^{2}\right)+\gamma_{c} \gamma_{p}\right]+2\left(A_{1} \varphi_{1}-\gamma_{p} \varphi_{2}\right)\right\}-\varphi_{2} . \\
2 L_{6} & {\left.\left[\gamma_{p}\left(\gamma_{c}+\gamma_{p}\right)+A_{2} \gamma_{c}\right]+2\left(\gamma_{p} \varphi_{1}-A_{2} \varphi_{2}\right)\right\} } \\
&
\end{aligned}
$$

(A.10) 
Proof of Proposition 7. Take the second-order partial derivatives of $\Pi_{m}$ with respect to $s_{m}, s_{z}$, and $\mu$ and obtain the Hessian matrix:

$$
H_{M}=\left(\begin{array}{ccc}
O_{2} & O_{3} & O_{4} \\
P_{2} & P_{3} & P_{4} \\
Q_{2} & Q_{3} & Q_{4}
\end{array}\right) .
$$

$\left|H_{1}\right|=O_{2},\left|H_{2}\right|=O_{2} P_{3}-O_{3} P_{2}$, and $\left|H_{3}\right|=O_{2}\left(P_{3} Q_{4}-\right.$ $\left.P_{4} Q_{3}\right)+O_{3}\left(P_{4} Q_{2}-P_{2} Q_{4}\right)+O_{4}\left(P_{2} Q_{3}-P_{3} Q_{2}\right)$. To ensure that $\Pi_{m}$ is jointly concave in $s_{m}, s_{z}$, and $\mu$, the parameters should meet the conditions that $\mathrm{O}_{2}<0, \mathrm{O}_{2} P_{3}-\mathrm{O}_{3} P_{2}->0$, and $\quad \mathrm{O}_{2}\left(P_{3} Q_{4}-P_{4} Q_{3}\right)+O_{3} \quad\left(P_{4} Q_{2}-P_{2} Q_{4}\right)+O_{4}$ $\left(P_{2} Q_{3}-P_{3} Q_{2}\right)<0$. The equilibrium decisions can be obtained where

$$
\begin{aligned}
& L_{7}=\frac{1}{2} c_{s}-\frac{L_{1}}{2 L_{6}}+\frac{L_{2}\left(L_{2} M_{1}+L_{1} M_{2}+c_{s} L_{6} M_{2}\right)}{4 L_{6}\left(2 \eta_{2} L_{6}+L_{2} M_{2}\right)} \text {, } \\
& L_{8}=\frac{L_{2}\left(L_{2} M_{3}+L_{3} M_{2}\right)}{4 L_{6}\left(2 \eta_{2} L_{6}+L_{2} M_{2}\right)}-\frac{L_{3}}{2 L_{6}}, \\
& L_{9}=\frac{L_{2}\left(L_{2} M_{4}+L_{4} M_{2}\right)}{4 L_{6}\left(2 \eta_{2} L_{6}+L_{2} M_{2}\right)}-\frac{L_{4}}{2 L_{6}}, \\
& L_{10}=\frac{L_{2}\left(L_{2} M_{5}+L_{5} M_{2}\right)}{4 L_{6}\left(2 \eta_{2} L_{6}+L_{2} M_{2}\right)}-\frac{L_{5}}{2 L_{6}}, \\
& L_{11}=\frac{2 A_{2} \alpha_{m}+2 \gamma_{p} \alpha_{z}-\gamma_{p} c_{s}\left(\gamma_{c}+3 \gamma_{p}\right)+2 A_{2} c_{s}\left(A_{1}-(1 / 2) \gamma_{c}\right)+3 L_{7}\left[\gamma_{p}\left(\gamma_{c}+\gamma_{p}\right)+A_{2} \gamma_{c}\right]}{4\left(A_{1} A_{2}-\gamma_{p}^{2}\right)} \\
& +\frac{\left(L_{2} M_{1}+L_{1} M_{2}+c_{s} L_{6} M_{2}\right)\left[\gamma_{p}\left(\theta_{c}+\theta_{p}\right)+A_{2} \theta_{c}\right]}{4\left(A_{1} A_{2}-\gamma_{p}^{2}\right)\left(2 \eta_{2} L_{6}+L_{2} M_{2}\right)} \\
& L_{12}=\frac{3 L_{8}\left[\gamma_{p}\left(\gamma_{c}+\gamma_{p}\right)+A_{2} \gamma_{c}\right]+2\left(A_{2} A_{3}-\gamma_{p} \theta_{p}\right)}{4\left(A_{1} A_{2}-\gamma_{p}^{2}\right)}+\frac{\left(L_{2} M_{3}+L_{3} M_{2}\right)\left[\gamma_{p}\left(\theta_{c}+\theta_{p}\right)+A_{2} \theta_{c}\right]}{4\left(A_{1} A_{2}-\gamma_{p}^{2}\right)\left(2 \eta_{2} L_{6}+L_{2} M_{2}\right)}, \\
& L_{13}=\frac{3 L_{9}\left[\gamma_{p}\left(\gamma_{c}+\gamma_{p}\right)+A_{2} \gamma_{c}\right]+2\left(\gamma_{p} A_{4}-A_{2} \theta_{p}\right)}{4\left(A_{1} A_{2}-\gamma_{p}^{2}\right)}+\frac{\left(L_{2} M_{4}+L_{4} M_{2}\right)\left[\gamma_{p}\left(\theta_{c}+\theta_{p}\right)+A_{2} \theta_{c}\right]}{4\left(A_{1} A_{2}-\gamma_{p}^{2}\right)\left(2 \eta_{2} L_{6}+L_{2} M_{2}\right)}, \\
& O_{1}=\frac{1}{2} L_{8}\left[\alpha_{r}-\left(A_{1}+\gamma_{c}\right) L_{7}+\gamma_{c} L_{11}+\left(\gamma_{c}+\gamma_{p}\right) L_{15}-\left(A_{3}+\theta_{c}\right) \frac{L_{2} M_{1}+L_{1} M_{2}+c_{s} L_{6} M_{2}}{4 \eta_{2} L_{6}+2 L_{2} M_{2}}\right] \\
& +\frac{1}{2}\left(L_{7}-c_{s}\right)\left[-\left(A_{1}+\gamma_{c}\right) L_{8}+\gamma_{c} L_{12}+\left(\gamma_{c}+\gamma_{p}\right) L_{16}-\left(A_{3}+\theta_{c}\right) \frac{L_{2} M_{3}+L_{3} M_{2}}{4 \eta_{2} L_{6}+2 L_{2} M_{2}}-\theta_{c}\right] \\
& +L_{12}\left[\alpha_{m}+\gamma_{c} L_{7}-A_{1} L_{11}+\gamma_{p} L_{15}+\theta_{c} \frac{L_{2} M_{1}+L_{1} M_{2}+c_{s} L_{6} M_{2}}{4 \eta_{2} L_{6}+2 L_{2} M_{2}}\right] \\
& +\left(L_{11}-c_{s}\right)\left(\gamma_{c} L_{8}-A_{1} L_{12}+\gamma_{p} L_{16}+\theta_{c} \frac{L_{2} M_{3}+L_{3} M_{2}}{4 \eta_{2} L_{6}+2 L_{2} M_{2}}+A_{3}\right)+L_{16}\left[\alpha_{z}+\left(\gamma_{c}+\gamma_{p}\right) L_{7}+\gamma_{p} L_{11}-A_{2} L_{15}\right. \\
& \left.+\left(\theta_{c}+\theta_{p}\right) \frac{L_{2} M_{1}+L_{1} M_{2}+c_{s} L_{6} M_{2}}{4 \eta_{2} L_{6}+2 L_{2} M_{2}}\right]+\left(L_{15}-c_{z}\right)\left[\left(\gamma_{c}+\gamma_{p}\right) L_{8}+\gamma_{p} L_{12}-A_{2} L_{16}+\left(\theta_{c}+\theta_{p}\right) \frac{L_{2} M_{3}+L_{3} M_{2}}{4 \eta_{2} L_{6}+2 L_{2} M_{2}}-\theta_{p}\right]
\end{aligned}
$$




$$
\begin{aligned}
& O_{2}=L_{8}\left[-\left(A_{1}+\gamma_{c}\right) L_{8}+\gamma_{c} L_{12}+\left(\gamma_{c}+\gamma_{p}\right) L_{16}-\left(A_{3}+\theta_{c}\right) \frac{L_{2} M_{3}+L_{3} M_{2}}{4 \eta_{2} L_{6}+2 L_{2} M_{2}}-\theta_{c}\right] \\
& +2 L_{12}\left(\gamma_{c} L_{8}-A_{1} L_{12}+\gamma_{p} L_{16}+\theta_{c} \frac{L_{2} M_{3}+L_{3} M_{2}}{4 \eta_{2} L_{6}+2 L_{2} M_{2}}+A_{3}\right) \\
& +2 L_{16}\left[\left(\gamma_{c}+\gamma_{p}\right) L_{8}+\gamma_{p} L_{12}-A_{2} L_{16}+\left(\theta_{c}+\theta_{p}\right) \frac{L_{2} M_{3}+L_{3} M_{2}}{4 \eta_{2} L_{6}+2 L_{2} M_{2}}-\theta_{p}\right]-\eta_{1} \text {, } \\
& O_{3}=\frac{1}{2} L_{8}\left[-\left(A_{1}+\gamma_{c}\right) L_{9}+\gamma_{c} L_{13}+\left(\gamma_{c}+\gamma_{p}\right) L_{17}-\left(A_{3}+\theta_{c}\right) \frac{L_{2} M_{4}+L_{4} M_{2}}{4 \eta_{2} L_{6}+2 L_{2} M_{2}}-\theta_{c}-\theta_{p}\right] \\
& +\frac{1}{2} L_{9}\left[-\left(A_{1}+\gamma_{c}\right) L_{8}+\gamma_{c} L_{12}+\left(\gamma_{c}+\gamma_{p}\right) L_{16}-\left(A_{3}+\theta_{c}\right) \frac{L_{2} M_{3}+L_{3} M_{2}}{4 \eta_{2} L_{6}+2 L_{2} M_{2}}-\theta_{c}\right] \\
& +L_{12}\left(\gamma_{c} L_{9}-A_{1} L_{13}+\gamma_{p} L_{17}+\theta_{c} \frac{L_{2} M_{4}+L_{4} M_{2}}{4 \eta_{2} L_{6}+2 L_{2} M_{2}}-\theta_{p}\right) \\
& +L_{13}\left(\gamma_{c} L_{8}-A_{1} L_{12}+\gamma_{p} L_{16}+\theta_{c} \frac{L_{2} M_{3}+L_{3} M_{2}}{4 \eta_{2} L_{6}+2 L_{2} M_{2}}+A_{3}\right) \\
& +L_{16}\left[\left(\gamma_{c}+\gamma_{p}\right) L_{9}+\gamma_{p} L_{13}-A_{2} L_{17}+\left(\theta_{c}+\theta_{p}\right) \frac{L_{2} M_{4}+L_{4} M_{2}}{4 \eta_{2} L_{6}+2 L_{2} M_{2}}+A_{4}\right]+L_{17}\left[\left(\gamma_{c}+\gamma_{p}\right) L_{8}+\gamma_{p} L_{12}-A_{2} L_{16}\right. \\
& \left.+\left(\theta_{c}+\theta_{p}\right) \frac{L_{2} M_{3}+L_{3} M_{2}}{4 \eta_{2} L_{6}+2 L_{2} M_{2}}-\theta_{p}\right] \\
& O_{4}=\frac{1}{2} L_{8}\left[-\left(A_{1}+\gamma_{c}\right) L_{10}+\gamma_{c} L_{14}+\left(\gamma_{c}+\gamma_{p}\right) L_{18}-\left(A_{3}+\theta_{c}\right) \frac{L_{2} M_{5}+L_{5} M_{2}}{4 \eta_{2} L_{6}+2 L_{2} M_{2}}-\varphi_{2}\right] \\
& +\frac{1}{2} L_{10}\left[-\left(A_{1}+\gamma_{c}\right) L_{8}+\gamma_{c} L_{12}+\left(\gamma_{c}+\gamma_{p}\right) L_{16}-\left(A_{3}+\theta_{c}\right) \frac{L_{2} M_{3}+L_{3} M_{2}}{4 \eta_{2} L_{6}+2 L_{2} M_{2}}-\theta_{c}\right] \\
& +L_{12}\left(\gamma_{c} L_{10}-A_{1} L_{14}+\gamma_{p} L_{18}+\theta_{c} \frac{L_{2} M_{5}+L_{5} M_{2}}{4 \eta_{2} L_{6}+2 L_{2} M_{2}}-\varphi_{2}\right)+L_{14}\left(\gamma_{c} L_{8}-A_{1} L_{12}+\gamma_{p} L_{16}+\theta_{c} \frac{L_{2} M_{3}+L_{3} M_{2}}{4 \eta_{2} L_{6}+2 L_{2} M_{2}}+A_{3}\right) \\
& +L_{16}\left[\left(\gamma_{c}+\gamma_{p}\right) L_{10}+\gamma_{p} L_{14}-A_{2} L_{18}\right. \\
& \left.+\left(\theta_{c}+\theta_{p}\right) \frac{L_{2} M_{5}+L_{5} M_{2}}{4 \eta_{2} L_{6}+2 L_{2} M_{2}}+\varphi_{1}\right]+L_{18}\left[\left(\gamma_{c}+\gamma_{p}\right) L_{8}+\gamma_{p} L_{12}-A_{2} L_{16}+\left(\theta_{c}+\theta_{p}\right) \frac{L_{2} M_{3}+L_{3} M_{2}}{4 \eta_{2} L_{6}+2 L_{2} M_{2}}-\theta_{p}\right] \text {, } \\
& P_{1}=\frac{1}{2} L_{9}\left[\alpha_{r}-\left(A_{1}+\gamma_{c}\right) L_{7}+\gamma_{c} L_{11}+\left(\gamma_{c}+\gamma_{p}\right) L_{15}-\left(A_{3}+\theta_{c}\right) \frac{L_{2} M_{1}+L_{1} M_{2}+c_{s} L_{6} M_{2}}{4 \eta_{2} L_{6}+2 L_{2} M_{2}}\right] \\
& +\frac{1}{2}\left(L_{7}-c_{s}\right)\left[-\left(A_{1}+\gamma_{c}\right) L_{9}+\gamma_{c} L_{13}+\left(\gamma_{c}+\gamma_{p}\right) L_{17}-\left(A_{3}+\theta_{c}\right) \frac{L_{2} M_{4}+L_{4} M_{2}}{4 \eta_{2} L_{6}+2 L_{2} M_{2}}-\theta_{c}-\theta_{p}\right] \\
& +L_{13}\left[\alpha_{m}+\gamma_{c} L_{7}-A_{1} L_{11}+\gamma_{p} L_{15}+\theta_{c} \frac{L_{2} M_{1}+L_{1} M_{2}+c_{s} L_{6} M_{2}}{4 \eta_{2} L_{6}+2 L_{2} M_{2}}\right] \\
& +\left(L_{11}-c_{s}\right)\left(\gamma_{c} L_{9}-A_{1} L_{13}+\gamma_{p} L_{17}+\theta_{c} \frac{L_{2} M_{4}+L_{4} M_{2}}{4 \eta_{2} L_{6}+2 L_{2} M_{2}}-\theta_{p}\right) \\
& +L_{17}\left[\alpha_{z}+\left(\gamma_{c}+\gamma_{p}\right) L_{7}+\gamma_{p} L_{11}-A_{2} L_{15}+\left(\theta_{c}+\theta_{p}\right) \frac{L_{2} M_{1}+L_{1} M_{2}+c_{s} L_{6} M_{2}}{4 \eta_{2} L_{6}+2 L_{2} M_{2}}\right] \\
& +\left(L_{15}-c_{z}\right)\left[\left(\gamma_{c}+\gamma_{p}\right) L_{9}+\gamma_{p} L_{13}-A_{2} L_{17}+\left(\theta_{c}+\theta_{p}\right) \frac{L_{2} M_{4}+L_{4} M_{2}}{4 \eta_{2} L_{6}+2 L_{2} M_{2}}+A_{4}\right] \text {, }
\end{aligned}
$$




$$
\begin{aligned}
& P_{2}=O_{3} \text {, } \\
& P_{3}=L_{9}\left[-\left(A_{1}+\gamma_{c}\right) L_{9}+\gamma_{c} L_{13}+\left(\gamma_{c}+\gamma_{p}\right) L_{17}-\left(A_{3}+\theta_{c}\right) \frac{L_{2} M_{4}+L_{4} M_{2}}{4 \eta_{2} L_{6}+2 L_{2} M_{2}}-\theta_{c}-\theta_{p}\right] \\
& +2 L_{13}\left(\gamma_{c} L_{9}-A_{1} L_{13}+\gamma_{p} L_{17}+\theta_{c} \frac{L_{2} M_{4}+L_{4} M_{2}}{4 \eta_{2} L_{6}+2 L_{2} M_{2}}-\theta_{p}\right)+2 L_{17}\left[\left(\gamma_{c}+\gamma_{p}\right)\right. \\
& \left.L_{9}+\gamma_{p} L_{13}-A_{2} L_{17}+\left(\theta_{c}+\theta_{p}\right) \frac{L_{2} M_{4}+L_{4} M_{2}}{4 \eta_{2} L_{6}+2 L_{2} M_{2}}+A_{4}\right]-\eta_{1} \text {, } \\
& P_{4}=\frac{1}{2} L_{9}\left[-\left(A_{1}+\gamma_{c}\right) L_{10}+\gamma_{c} L_{14}+\left(\gamma_{c}+\gamma_{p}\right) L_{18}-\left(A_{3}+\theta_{c}\right) \frac{L_{2} M_{5}+L_{5} M_{2}}{4 \eta_{2} L_{6}+2 L_{2} M_{2}}-\varphi_{2}\right] \\
& +\frac{1}{2} L_{10}\left[-\left(A_{1}+\gamma_{c}\right) L_{9}+\gamma_{c} L_{13}+\left(\gamma_{c}+\gamma_{p}\right) L_{17}-\left(A_{3}+\theta_{c}\right) \frac{L_{2} M_{4}+L_{4} M_{2}}{4 \eta_{2} L_{6}+2 L_{2} M_{2}}-\theta_{c}-\theta_{p}\right] \\
& +L_{13}\left(\gamma_{c} L_{10}-A_{1} L_{14}+\gamma_{p} L_{18}+\theta_{c} \frac{L_{2} M_{5}+L_{5} M_{2}}{4 \eta_{2} L_{6}+2 L_{2} M_{2}}-\varphi_{2}\right) \\
& +L_{14}\left(\gamma_{c} L_{9}-A_{1} L_{13}+\gamma_{p} L_{17}+\theta_{c} \frac{L_{2} M_{4}+L_{4} M_{2}}{4 \eta_{2} L_{6}+2 L_{2} M_{2}}-\theta_{p}\right) \\
& +L_{17}\left[\left(\gamma_{c}+\gamma_{p}\right) L_{10}+\gamma_{p} L_{14}-A_{2} L_{18}+\left(\theta_{c}+\theta_{p}\right) \frac{L_{2} M_{5}+L_{5} M_{2}}{4 \eta_{2} L_{6}+2 L_{2} M_{2}}+\varphi_{1}\right] \\
& +L_{18}\left[\left(\gamma_{c}+\gamma_{p}\right) L_{9}+\gamma_{p} L_{13}-A_{2} L_{17}+\left(\theta_{c}+\theta_{p}\right) \frac{L_{2} M_{4}+L_{4} M_{2}}{4 \eta_{2} L_{6}+2 L_{2} M_{2}}+A_{4}\right] \text {, } \\
& Q_{1}=\frac{1}{2} L_{10}\left[\alpha_{r}-\left(A_{1}+\gamma_{c}\right) L_{7}+\gamma_{c} L_{11}+\left(\gamma_{c}+\gamma_{p}\right) L_{15}-\left(A_{3}+\theta_{c}\right) \frac{L_{2} M_{1}+L_{1} M_{2}+c_{s} L_{6} M_{2}}{4 \eta_{2} L_{6}+2 L_{2} M_{2}}\right] \\
& +\frac{1}{2}\left(L_{7}-c_{s}\right)\left[-\left(A_{1}+\gamma_{c}\right) L_{10}+\gamma_{c} L_{14}+\left(\gamma_{c}+\gamma_{p}\right) L_{18}-\left(A_{3}+\theta_{c}\right) \frac{L_{2} M_{5}+L_{5} M_{2}}{4 \eta_{2} L_{6}+2 L_{2} M_{2}}-\varphi_{2}\right] \\
& +L_{14}\left[\alpha_{m}+\gamma_{c} L_{7}-A_{1} L_{11}+\gamma_{p} L_{15}+\theta_{c} \frac{L_{2} M_{1}+L_{1} M_{2}+c_{s} L_{6} M_{2}}{4 \eta_{2} L_{6}+2 L_{2} M_{2}}\right]+\left(L_{11}-c_{s}\right) \\
& \cdot\left(\gamma_{c} L_{10}-A_{1} L_{14}+\gamma_{p} L_{18}+\theta_{c} \frac{L_{2} M_{5}+L_{5} M_{2}}{4 \eta_{2} L_{6}+2 L_{2} M_{2}}-\varphi_{2}\right)+L_{18}\left[\alpha_{z}+\left(\gamma_{c}+\gamma_{p}\right) L_{7}+\gamma_{p} L_{11}-A_{2} L_{15}\right. \\
& \left.+\left(\theta_{c}+\theta_{p}\right) \frac{L_{2} M_{1}+L_{1} M_{2}+c_{s} L_{6} M_{2}}{4 \eta_{2} L_{6}+2 L_{2} M_{2}}\right]+\left(L_{15}-c_{z}\right)\left[\left(\gamma_{c}+\gamma_{p}\right) L_{10}+\gamma_{p} L_{14}-A_{2} L_{18}+\left(\theta_{c}+\theta_{p}\right)\right. \\
& \left.\frac{L_{2} M_{5}+L_{5} M_{2}}{4 \eta_{2} L_{6}+2 L_{2} M_{2}}+\varphi_{1}\right]
\end{aligned}
$$




$$
\begin{aligned}
& Q_{2}=\frac{1}{2} L_{10}\left[-\left(A_{1}+\gamma_{c}\right) L_{8}+\gamma_{c} L_{12}+\left(\gamma_{c}+\gamma_{p}\right) L_{16}-\left(A_{3}+\theta_{c}\right) \frac{L_{2} M_{3}+L_{3} M_{2}}{4 \eta_{2} L_{6}+2 L_{2} M_{2}}-\theta_{c}\right] \\
& +\frac{1}{2} L_{8}\left[-\left(A_{1}+\gamma_{c}\right) L_{10}+\gamma_{c} L_{14}+\left(\gamma_{c}+\gamma_{p}\right) L_{18}-\left(A_{3}+\theta_{c}\right) \frac{L_{2} M_{5}+L_{5} M_{2}}{4 \eta_{2} L_{6}+2 L_{2} M_{2}}-\varphi_{2}\right] \\
& +L_{14}\left(\gamma_{c} L_{8}-A_{1} L_{12}+\gamma_{p} L_{16}+\theta_{c} \frac{L_{2} M_{3}+L_{3} M_{2}}{4 \eta_{2} L_{6}+2 L_{2} M_{2}}+A_{3}\right)+L_{12}\left(\gamma_{c} L_{10}-A_{1} L_{14}+\gamma_{p} L_{18}+\theta_{c} \frac{L_{2} M_{5}+L_{5} M_{2}}{4 \eta_{2} L_{6}+2 L_{2} M_{2}}-\varphi_{2}\right) \\
& +L_{18}\left[\left(\gamma_{c}+\gamma_{p}\right) L_{8}+\gamma_{p} L_{12}-A_{2} L_{16}+\left(\theta_{c}+\theta_{p}\right) \frac{L_{2} M_{3}+L_{3} M_{2}}{4 \eta_{2} L_{6}+2 L_{2} M_{2}}-\theta_{p}\right] \\
& +L_{16}\left[\left(\gamma_{c}+\gamma_{p}\right) L_{10}+\gamma_{p} L_{14}-A_{2} L_{18}+\left(\theta_{c}+\theta_{p}\right) \frac{L_{2} M_{5}+L_{5} M_{2}}{4 \eta_{2} L_{6}+2 L_{2} M_{2}}+\varphi_{1}\right] \text {, } \\
& Q_{2}=P_{4} \text {, } \\
& Q_{4}=L_{10}\left[-\left(A_{1}+\gamma_{c}\right) L_{10}+\gamma_{c} L_{14}+\left(\gamma_{c}+\gamma_{p}\right) L_{18}-\left(A_{3}+\theta_{c}\right) \frac{L_{2} M_{5}+L_{5} M_{2}}{4 \eta_{2} L_{6}+2 L_{2} M_{2}}-\varphi_{2}\right] \\
& +2 L_{14}\left(\gamma_{c} L_{10}-A_{1} L_{14}+\gamma_{p} L_{18}+\theta_{c} \frac{L_{2} M_{5}+L_{5} M_{2}}{4 \eta_{2} L_{6}+2 L_{2} M_{2}}-\varphi_{2}\right)+2 L_{18}\left[\left(\gamma_{c}+\gamma_{p}\right) L_{10}+\gamma_{p} L_{14}-A_{2} L_{18}+\left(\theta_{c}+\theta_{p}\right)\right. \\
& \left.\frac{L_{2} M_{5}+L_{5} M_{2}}{4 \eta_{2} L_{6}+2 L_{2} M_{2}}+\varphi_{1}\right]-C
\end{aligned}
$$

Proof of Proposition 8. Take the second-order partial derivatives of $\Pi_{m}$ with respect to $p_{m}$ and $p_{z}$ to obtain the Hessian matrix:

$$
H_{M}=\left(\begin{array}{cc}
-2 A_{1} & 2 \gamma_{p} \\
2 \gamma_{p} & -2 A_{2}
\end{array}\right)
$$

$\left|H_{1}\right|=-2 A_{1}<0$ and $\left|H_{2}\right|=4 A_{1} A_{2}-4 \gamma_{p}^{2}>0$. The profit of the manufacturer is jointly concave in $p_{m}$ and $p_{z}$. $\left(\partial^{2} \Pi_{r} / \partial p_{r}^{2}\right)=-2\left(A_{1}+\gamma_{c}\right)<0$, so that the profit of the retailer is concave in $p_{r}$.

Take $\left(\partial \Pi_{m} / \partial p_{m}\right)=0,\left(\partial \Pi_{m} / \partial p_{z}\right)=0$, and $\left(\partial \Pi_{r} / \partial p_{r}\right)=$ 0 and obtain the equilibrium solutions as follows: $p_{r}^{*}=\left(R_{1}+R_{2} s_{r}+R_{3} s_{m}+R_{4} s_{z}+R_{5} \mu / R_{6}\right), p_{m}^{*}=\left(T_{1}+T_{2} s_{r}+\right.$ $\left.T_{3} s_{m}+T_{4} s_{z}+T_{5} \mu / R_{6}\right)$, and $p_{z}^{*}=\left(U_{1}+U_{2} s_{r}+U_{3} s_{m}+\right.$ $\left.U_{4} s_{z}+U_{5} \mu / R_{6}\right)$ where

$$
\begin{aligned}
R_{1}= & -\left[\gamma_{p}\left(\gamma_{c}+\gamma_{p}\right)+A_{2} \gamma_{c}\right]\left[\alpha_{m}+c_{s}\left(A_{1}-\frac{1}{2} \gamma_{c}\right)-c_{z} \gamma_{p}\right]-\left[\gamma_{c} \gamma_{p}+A_{1}\left(\gamma_{c}+\gamma_{p}\right)\right] \\
& \cdot\left[\alpha_{z}-\frac{1}{2} c_{s}\left(\gamma_{c}+3 \gamma_{p}\right)+c_{z} A_{2}\right]+2\left(\gamma_{p}^{2}-A_{1} A_{2}\right)\left[\alpha_{r}+c_{s}\left(A_{1}+\gamma_{c}\right)\right], \\
R_{2}= & \theta_{c}\left[\gamma_{p}\left(\gamma_{c}+\gamma_{p}\right)+A_{2} \gamma_{c}\right]+\left(\theta_{c}+\theta_{p}\right)\left[\gamma_{c} \gamma_{p}+A_{1}\left(\gamma_{c}+\gamma_{p}\right)\right]+2\left(\gamma_{p}^{2}-A_{1} A_{2}\right)\left(A_{3}+\theta_{c}\right), \\
R_{3}= & -A_{3}\left[\gamma_{p}\left(\gamma_{c}+\gamma_{p}\right)+A_{2} \gamma_{c}\right]+\theta_{p}\left[\gamma_{c} \gamma_{p}+A_{1}\left(\gamma_{c}+\gamma_{p}\right)\right]-2 \theta_{c}\left(\gamma_{p}^{2}-A_{1} A_{2}\right), \\
R_{4}= & \theta_{p}\left[\gamma_{p}\left(\gamma_{c}+\gamma_{p}\right)+A_{2} \gamma_{c}\right]-A_{4}\left[\gamma_{c} \gamma_{p}+A_{1}\left(\gamma_{c}+\gamma_{p}\right)\right]-2\left(\theta_{c}+\theta_{p}\right)\left(\gamma_{p}^{2}-A_{1} A_{2}\right) \\
R_{5}= & \varphi_{2}\left[\gamma_{p}\left(\gamma_{c}+\gamma_{p}\right)+A_{2} \gamma_{c}\right]-\varphi_{1}\left[\gamma_{c} \gamma_{p}+A_{1}\left(\gamma_{c}+\gamma_{p}\right)\right]-2 \varphi_{2}\left(\gamma_{p}^{2}-A_{1} A_{2}\right), \\
R_{6}= & 3 \gamma_{c} \gamma_{p}\left(\gamma_{c}+\gamma_{p}\right)+\frac{3}{2} \gamma_{c}^{2} A_{2}+\frac{3}{2}\left(\gamma_{c}+\gamma_{p}\right)^{2} A_{1}+4\left(\gamma_{p}^{2}-A_{1} A_{2}\right)\left(A_{1}+\gamma_{c}\right),
\end{aligned}
$$




$$
\begin{aligned}
& T_{1}=\left[\frac{3}{4}\left(\gamma_{c}+\gamma_{p}\right)^{2}-2 A_{2}\left(A_{1}+\gamma_{c}\right)\right]\left[\alpha_{m}+c_{s}\left(A_{1}-\frac{1}{2} \gamma_{c}\right)-c_{z} \gamma_{p}\right]-\left[2 \gamma_{p}\left(A_{1}+\gamma_{c}\right)+\frac{3}{4} \gamma_{c}\left(\gamma_{c}+\gamma_{p}\right)\right] \\
& \cdot\left[\alpha_{z}-\frac{1}{2} c_{s}\left(\gamma_{c}+3 \gamma_{p}\right)+c_{z} A_{2}\right]-\frac{3}{2}\left[\gamma_{c} A_{2}+\gamma_{p}\left(\gamma_{c}+\gamma_{p}\right)\right]\left[\alpha_{r}+c_{s}\left(A_{1}+\gamma_{c}\right)\right] \\
& T_{2}=-\theta_{c}\left[\frac{3}{4}\left(\gamma_{c}+\gamma_{p}\right)^{2}-2 A_{2}\left(A_{1}+\gamma_{c}\right)\right]+\left(\theta_{c}+\theta_{p}\right) \\
& \cdot\left[2 \gamma_{p}\left(A_{1}+\gamma_{c}\right)+\frac{3}{4} \gamma_{c}\left(\gamma_{c}+\gamma_{p}\right)\right]-\frac{3}{2}\left(A_{3}+\theta_{c}\right)\left[\gamma_{c} A_{2}+\gamma_{p}\left(\gamma_{c}+\gamma_{p}\right)\right] \\
& T_{3}=A_{3}\left[\frac{3}{4}\left(\gamma_{c}+\gamma_{p}\right)^{2}-2 A_{2}\left(A_{1}+\gamma_{c}\right)\right]+\theta_{p}\left[2 \gamma_{p}\left(A_{1}+\gamma_{c}\right)+\frac{3}{4} \gamma_{c}\left(\gamma_{c}+\gamma_{p}\right)\right]-\frac{3}{2} \theta_{c}\left[\gamma_{c} A_{2}+\gamma_{p}\left(\gamma_{c}+\gamma_{p}\right)\right] \\
& T_{4}=-\theta_{p}\left[\frac{3}{4}\left(\gamma_{c}+\gamma_{p}\right)^{2}-2 A_{2}\left(A_{1}+\gamma_{c}\right)\right]-A_{4}\left[2 \gamma_{p}\left(A_{1}+\gamma_{c}\right)+\frac{3}{4} \gamma_{c}\left(\gamma_{c}+\gamma_{p}\right)\right]+\frac{3}{2}\left(\theta_{c}+\theta_{p}\right)\left[\gamma_{c} A_{2}+\gamma_{p}\left(\gamma_{c}+\gamma_{p}\right)\right] \text {, } \\
& T_{5}=-\varphi_{2}\left[\frac{3}{4}\left(\gamma_{c}+\gamma_{p}\right)^{2}-2 A_{2}\left(A_{1}+\gamma_{c}\right)\right]-\varphi_{1}\left[2 \gamma_{p}\left(A_{1}+\gamma_{c}\right)+\frac{3}{4} \gamma_{c}\left(\gamma_{c}+\gamma_{p}\right)\right]+\frac{3}{2} \varphi_{2}\left[\gamma_{c} A_{2}+\gamma_{p}\left(\gamma_{c}+\gamma_{p}\right)\right] \\
& U_{1}=-\left[2 \gamma_{p}\left(A_{1}+\gamma_{c}\right)+\frac{3}{4} \gamma_{c}\left(\gamma_{c}+\gamma_{p}\right)\right]\left[\alpha_{m}+c_{s}\left(A_{1}-\frac{1}{2} \gamma_{c}\right)-c_{z} \gamma_{p}\right]+\left[\frac{3}{4} \gamma_{c}^{2}-2 A_{1}\left(A_{1}+\gamma_{c}\right)\right] \\
& \cdot\left[\alpha_{z}-\frac{1}{2} c_{s}\left(\gamma_{c}+3 \gamma_{p}\right)+c_{z} A_{2}\right]-\frac{3}{2}\left[A_{1}\left(\gamma_{c}+\gamma_{p}\right)+\gamma_{c} \gamma_{p}\right]\left[\alpha_{r}+c_{s}\left(A_{1}+\gamma_{c}\right)\right] \text {, } \\
& U_{2}=\theta_{c}\left[2 \gamma_{p}\left(A_{1}+\gamma_{c}\right)+\frac{3}{4} \gamma_{c}\left(\gamma_{c}+\gamma_{p}\right)\right]-\left(\theta_{c}+\theta_{p}\right)\left[\frac{3}{4} \gamma_{c}^{2}-2 A_{1}\left(A_{1}+\gamma_{c}\right)\right]-\frac{3}{2}\left(A_{3}+\theta_{c}\right)\left[A_{1}\left(\gamma_{c}+\gamma_{p}\right)+\gamma_{c} \gamma_{p}\right] \text {, } \\
& U_{3}=-A_{3}\left[2 \gamma_{p}\left(A_{1}+\gamma_{c}\right)+\frac{3}{4} \gamma_{c}\left(\gamma_{c}+\gamma_{p}\right)\right]-\theta_{p}\left[\frac{3}{4} \gamma_{c}^{2}-2 A_{1}\left(A_{1}+\gamma_{c}\right)\right]+\frac{3}{2} \theta_{c}\left[A_{1}\left(\gamma_{c}+\gamma_{p}\right)+\gamma_{c} \gamma_{p}\right] \text {, } \\
& U_{4}=\theta_{p}\left[2 \gamma_{p}\left(A_{1}+\gamma_{c}\right)+\frac{3}{4} \gamma_{c}\left(\gamma_{c}+\gamma_{p}\right)\right]+A_{4}\left[\frac{3}{4} \gamma_{c}^{2}-2 A_{1}\left(A_{1}+\gamma_{c}\right)\right]+\frac{3}{2}\left(\theta_{c}+\theta_{p}\right)\left[A_{1}\left(\gamma_{c}+\gamma_{p}\right)+\gamma_{c} \gamma_{p}\right] \text {, } \\
& U_{5}=\varphi_{2}\left[2 \gamma_{p}\left(A_{1}+\gamma_{c}\right)+\frac{3}{4} \gamma_{c}\left(\gamma_{c}+\gamma_{p}\right)\right]+\varphi_{1}\left[\frac{3}{4} \gamma_{c}^{2}-2 A_{1}\left(A_{1}+\gamma_{c}\right)\right]+\frac{3}{2} \varphi_{2}\left[A_{1}\left(\gamma_{c}+\gamma_{p}\right)+\gamma_{c} \gamma_{p}\right]
\end{aligned}
$$

Proof of Proposition 9. Take the second-order partial derivatives of $\Pi_{r}$ with respect to $p_{r},\left(\partial^{2} \Pi_{r} / \partial s_{r}^{2}\right)=\left(R_{2} / R_{6}\right)\left[A_{3}+\right.$ $\left.\theta_{c}+\left(-R_{2}\left(A_{1}+\gamma_{c}\right)+T_{2} \gamma_{c}+U_{2}\left(\gamma_{c}+\gamma_{p}\right) / R_{6}\right)\right]-\eta_{2}$. To ensure that $\Pi_{r}$ is concave in $s_{r}, \partial^{2} \prod_{r} / \partial s_{r}^{2}$ should be negative.
Therefore, the optimal $s_{r}$ can be obtained provided that $\eta_{2}>\left(R_{2} / R_{6}\right)\left[A_{3}+\theta_{c}+\left(-R_{2}\right.\right.$

$\left.\left.\left(A_{1}+\gamma_{c}\right)+T_{2} \gamma_{c}+U_{2}\left(\gamma_{c}+\gamma_{p}\right) / R_{6}\right)\right]>$, where

$$
\begin{aligned}
& U_{6}=\frac{R_{2}}{2 R_{6}}\left[\alpha_{r}+\frac{-R_{1}\left(A_{1}+\gamma_{c}\right)+T_{1} \gamma_{c}+U_{1}\left(\gamma_{c}+\gamma_{p}\right)}{R_{6}}\right]+\frac{1}{2}\left(\frac{R_{1}}{R_{6}}-c_{s}\right)\left[A_{3}+\theta_{c}+\frac{-R_{2}\left(A_{1}+\gamma_{c}\right)+T_{2} \gamma_{c}+U_{2}\left(\gamma_{c}+\gamma_{p}\right)}{R_{6}}\right], \\
& U_{7}=\frac{R_{2}}{2 R_{6}}\left[-\theta_{c}+\frac{-R_{3}\left(A_{1}+\gamma_{c}\right)+T_{3} \gamma_{c}+U_{3}\left(\gamma_{c}+\gamma_{p}\right)}{R_{6}}\right]+\frac{R_{3}}{2 R_{6}}\left[A_{3}+\theta_{c}+\frac{-R_{2}\left(A_{1}+\gamma_{c}\right)+T_{2} \gamma_{c}+U_{2}\left(\gamma_{c}+\gamma_{p}\right)}{R_{6}}\right], \\
& U_{8}=\frac{R_{2}}{2 R_{6}}\left[-\theta_{c}-\theta_{p}+\frac{-R_{4}\left(A_{1}+\gamma_{c}\right)+T_{4} \gamma_{c}+U_{4}\left(\gamma_{c}+\gamma_{p}\right)}{R_{6}}\right]+\frac{R_{4}}{2 R_{6}}\left[A_{3}+\theta_{c}+\frac{-R_{2}\left(A_{1}+\gamma_{c}\right)+T_{2} \gamma_{c}+U_{2}\left(\gamma_{c}+\gamma_{p}\right)}{R_{6}}\right], \\
& U_{9}=\frac{R_{2}}{2 R_{6}}\left[-\varphi_{2}+\frac{-R_{5}\left(A_{1}+\gamma_{c}\right)+T_{5} \gamma_{c}+U_{5}\left(\gamma_{c}+\gamma_{p}\right)}{R_{6}}\right]+\frac{R_{5}}{2 R_{6}}\left[A_{3}+\theta_{c}+\frac{-R_{2}\left(A_{1}+\gamma_{c}\right)+T_{2} \gamma_{c}+U_{2}\left(\gamma_{c}+\gamma_{p}\right)}{R_{6}}\right], \\
& U_{10}=\eta_{2}-\frac{R_{2}}{R_{6}}\left[A_{3}+\theta_{c}+\frac{-R_{2}\left(A_{1}+\gamma_{c}\right)+T_{2} \gamma_{c}+U_{2}\left(\gamma_{c}+\gamma_{p}\right)}{R_{6}}\right]=+.
\end{aligned}
$$


Proof of Proposition 10. Take the second-order partial derivatives of $\Pi_{m}$ with respect to $s_{m}, s_{z}$, and $\mu$ and obtain the Hessian matrix:

$$
H_{M}=\left(\begin{array}{ccc}
V_{2} & V_{3} & V_{4} \\
X_{2} & X_{3} & X_{4} \\
Y_{2} & Y_{3} & Y_{4}
\end{array}\right) .
$$

$\left|H_{1}\right|=V_{2},\left|H_{2}\right|=V_{2} X_{3}-V_{3} X_{2}$, and $\left|H_{3}\right|=V_{2}\left(X_{3} Y_{4}-\right.$ $\left.X_{4} Y_{3}\right)+V_{3}\left(X_{4} Y_{2}-X_{2} Y_{4}\right)+V_{4}\left(X_{2} Y_{3}-X_{3} Y_{2}\right)$. To ensure that $\Pi_{m}$ is jointly concave in $s_{m}, s_{z}$, and $\mu$, the parameters should meet the conditions that $V_{2}<0, V_{2} X_{3}-V_{3} X_{2}->0$, and $\quad V_{2}\left(X_{3} Y_{4}-X_{4} Y_{3}\right)+V_{3} \quad\left(X_{4} Y_{2}-X_{2} Y_{4}\right)+V_{4}$ $\left(X_{2} Y_{3}-X_{3} Y_{2}\right)<0$. The equilibrium decisions can be obtained where

$$
\begin{aligned}
& V_{1}=\frac{R_{3} U_{10}+R_{2} U_{7}}{2 R_{6} U_{10}}\left[\alpha_{r}+\frac{-\left(A_{1}+\gamma_{c}\right)\left(R_{1} U_{10}+R_{2} U_{6}\right)+\gamma_{c}\left(T_{1} U_{10}+T_{2} U_{6}\right)+\left(\gamma_{c}+\gamma_{p}\right)\left(U_{1} U_{10}+U_{2} U_{6}\right)}{R_{6} U_{10}}+\frac{U_{6}\left(A_{3}+\theta_{c}\right)}{U_{10}}\right] \\
& +\frac{1}{2}\left(\frac{R_{1} U_{10}+R_{2} U_{6}}{R_{6} U_{10}}-c_{s}\right) \\
& \left.\frac{-\left(A_{1}+\gamma_{c}\right)\left(R_{3} U_{10}+R_{2} U_{7}\right)+\gamma_{c}\left(T_{3} U_{10}+T_{2} U_{7}\right)+\left(\gamma_{c}+\gamma_{p}\right)\left(U_{3} U_{10}+U_{2} U_{7}\right)}{R_{6} U_{10}}+\frac{U_{7}\left(A_{3}+\theta_{c}\right)}{U_{10}}-\theta_{c}\right] \\
& \left.+\frac{T_{3} U_{10}+T_{2} U_{7}}{R_{6} U_{10}} \alpha_{m}+\frac{\gamma_{c}\left(R_{1} U_{10}+R_{2} U_{6}\right)-A_{1}\left(T_{1} U_{10}+T_{2} U_{6}\right)+\gamma_{p}\left(U_{1} U_{10}+U_{2} U_{6}\right)}{R_{6} U_{10}}-\frac{U_{6} \theta_{c}}{U_{10}}\right] \\
& +\left(\frac{T_{1} U_{10}+T_{2} U_{6}}{R_{6} U_{10}}-c_{s}\right)\left[\frac{\gamma_{c}\left(R_{3} U_{10}+R_{2} U_{7}\right)-A_{1}\left(T_{3} U_{10}+T_{2} U_{7}\right)+\gamma_{p}\left(U_{3} U_{10}+U_{2} U_{7}\right)}{R_{6} U_{10}}-\frac{U_{7} \theta_{c}}{U_{10}}+A_{3}\right] \\
& +\frac{U_{3} U_{10}+U_{2} U_{7}}{R_{6} U_{10}}\left[\alpha_{z}+\frac{\left(\gamma_{c}+\gamma_{p}\right)\left(R_{1} U_{10}+R_{2} U_{6}\right)+\gamma_{p}\left(T_{1} U_{10}+T_{2} U_{6}\right)-A_{2}\left(U_{1} U_{10}+U_{2} U_{6}\right)}{R_{6} U_{10}}-\frac{U_{6}\left(\theta_{c}+\theta_{p}\right)}{U_{10}}\right] \\
& +\left(\frac{U_{1} U_{10}+U_{2} U_{6}}{R_{6} U_{10}}-c_{z}\right),\left[\frac{\left(\gamma_{c}+\gamma_{p}\right)\left(R_{3} U_{10}+R_{2} U_{7}\right)+\gamma_{p}\left(T_{3} U_{10}+T_{2} U_{7}\right)-A_{2}\left(U_{3} U_{10}+U_{2} U_{7}\right)}{R_{6} U_{10}}-\frac{U_{7}\left(\theta_{c}+\theta_{p}\right)}{U_{10}}-\theta_{p}\right], \\
& V_{2}=\frac{R_{3} U_{10}+R_{2} U_{7}}{R_{6} U_{10}}\left[\frac{-\left(A_{1}+\gamma_{c}\right)\left(R_{3} U_{10}+R_{2} U_{7}\right)+\gamma_{c}\left(T_{3} U_{10}+T_{2} U_{7}\right)+\left(\gamma_{c}+\gamma_{p}\right)\left(U_{3} U_{10}+U_{2} U_{7}\right)}{R_{6} U_{10}}+\frac{U_{7}\left(A_{3}+\theta_{c}\right)}{U_{10}}-\theta_{c}\right] \\
& +\frac{2\left(T_{3} U_{10}+T_{2} U_{7}\right)}{R_{6} U_{10}}\left[\frac{\gamma_{c}\left(R_{3} U_{10}+R_{2} U_{7}\right)-A_{1}\left(T_{3} U_{10}+T_{2} U_{7}\right)+\gamma_{p}\left(U_{3} U_{10}+U_{2} U_{7}\right)}{R_{6} U_{10}}-\frac{U_{7} \theta_{c}}{U_{10}}+A_{3}\right] \\
& +\frac{2\left(U_{3} U_{10}+U_{2} U_{7}\right)}{R_{6} U_{10}}\left[\frac{\left(\gamma_{c}+\gamma_{p}\right)\left(R_{3} U_{10}+R_{2} U_{7}\right)+\gamma_{p}\left(T_{3} U_{10}+T_{2} U_{7}\right)-A_{2}\left(U_{3} U_{10}+U_{2} U_{7}\right)}{R_{6} U_{10}}-\frac{U_{7}\left(\theta_{c}+\theta_{p}\right)}{U_{10}}-\theta_{p}\right]-\eta_{1} \text {, } \\
& V_{3}=\frac{R_{3} U_{10}+R_{2} U_{7}}{2 R_{6} U_{10}}\left[\frac{-\left(A_{1}+\gamma_{c}\right)\left(R_{4} U_{10}+R_{2} U_{8}\right)+\gamma_{c}\left(T_{4} U_{10}+T_{2} U_{8}\right)+\left(\gamma_{c}+\gamma_{p}\right)\left(U_{4} U_{10}+U_{2} U_{8}\right)}{R_{6} U_{10}}+\frac{U_{8}\left(A_{3}+\theta_{c}\right)}{U_{10}}-\theta_{c}-\theta_{p}\right] \\
& +\frac{R_{4} U_{10}+R_{2} U_{8}}{2 R_{6} U_{10}}\left[\frac{-\left(A_{1}+\gamma_{c}\right)\left(R_{3} U_{10}+R_{2} U_{7}\right)+\gamma_{c}\left(T_{3} U_{10}+T_{2} U_{7}\right)+\left(\gamma_{c}+\gamma_{p}\right)\left(U_{3} U_{10}+U_{2} U_{7}\right)}{R_{6} U_{10}}+\frac{U_{7}\left(A_{3}+\theta_{c}\right)}{U_{10}}-\theta_{c}\right] \\
& +\frac{T_{3} U_{10}+T_{2} U_{7}}{R_{6} U_{10}}\left[\frac{\gamma_{c}\left(R_{4} U_{10}+R_{2} U_{8}\right)-A_{1}\left(T_{4} U_{10}+T_{2} U_{8}\right)+\gamma_{p}\left(U_{4} U_{10}+U_{2} U_{8}\right)}{R_{6} U_{10}}-\frac{U_{8} \theta_{c}}{U_{10}}-\theta_{p}\right]+\frac{T_{4} U_{10}+T_{2} U_{8}}{R_{6} U_{10}}
\end{aligned}
$$




$$
\begin{aligned}
& \cdot\left[\frac{\gamma_{c}\left(R_{3} U_{10}+R_{2} U_{7}\right)-A_{1}\left(T_{3} U_{10}+T_{2} U_{7}\right)+\gamma_{p}\left(U_{3} U_{10}+U_{2} U_{7}\right)}{R_{6} U_{10}}-\frac{U_{7} \theta_{c}}{U_{10}}+A_{3}\right]+\frac{U_{3} U_{10}+U_{2} U_{7}}{R_{6} U_{10}} \\
& \cdot\left[\frac{\left(\gamma_{c}+\gamma_{p}\right)\left(R_{4} U_{10}+R_{2} U_{8}\right)+\gamma_{p}\left(T_{4} U_{10}+T_{2} U_{8}\right)-A_{2}\left(U_{4} U_{10}+U_{2} U_{8}\right)}{R_{6} U_{10}}-\frac{U_{8}\left(\theta_{c}+\theta_{p}\right)}{U_{10}}+A_{4}\right] \\
& +\frac{U_{4} U_{10}+U_{2} U_{8}}{R_{6} U_{10}}\left[\frac{\left(\gamma_{c}+\gamma_{p}\right)\left(R_{3} U_{10}+R_{2} U_{7}\right)+\gamma_{p}\left(T_{3} U_{10}+T_{2} U_{7}\right)-A_{2}\left(U_{3} U_{10}+U_{2} U_{7}\right)}{R_{6} U_{10}}-\frac{U_{7}\left(\theta_{c}+\theta_{p}\right)}{U_{10}}-\theta_{p}\right], \\
& V_{4}=\frac{R_{3} U_{10}+R_{2} U_{7}}{2 R_{6} U_{10}}\left[\frac{-\left(A_{1}+\gamma_{c}\right)\left(R_{5} U_{10}+R_{2} U_{9}\right)+\gamma_{c}\left(T_{5} U_{10}+T_{2} U_{9}\right)+\left(\gamma_{c}+\gamma_{p}\right)\left(U_{5} U_{10}+U_{2} U_{9}\right)}{R_{6} U_{10}}+\frac{U_{9}\left(A_{3}+\theta_{c}\right)}{U_{10}}-\varphi_{2}\right] \\
& +\frac{R_{5} U_{10}+R_{2} U_{9}}{2 R_{6} U_{10}} \\
& \cdot\left[\frac{-\left(A_{1}+\gamma_{c}\right)\left(R_{3} U_{10}+R_{2} U_{7}\right)+\gamma_{c}\left(T_{3} U_{10}+T_{2} U_{7}\right)+\left(\gamma_{c}+\gamma_{p}\right)\left(U_{3} U_{10}+U_{2} U_{7}\right)}{R_{6} U_{10}}+\frac{U_{7}\left(A_{3}+\theta_{c}\right)}{U_{10}}-\theta_{c}\right]+\frac{T_{3} U_{10}+T_{2} U_{7}}{R_{6} U_{10}} \\
& \cdot\left[\frac{\gamma_{c}\left(R_{5} U_{10}+R_{2} U_{9}\right)-A_{1}\left(T_{5} U_{10}+T_{2} U_{9}\right)+\gamma_{p}\left(U_{5} U_{10}+U_{2} U_{9}\right)}{R_{6} U_{10}}-\frac{U_{9} \theta_{c}}{U_{10}}-\varphi_{2}\right]+\frac{T_{5} U_{10}+T_{2} U_{9}}{R_{6} U_{10}}+\frac{U_{3} U_{10}+U_{2} U_{7}}{R_{6} U_{10}} \\
& \cdot\left[\frac{\gamma_{c}\left(R_{3} U_{10}+R_{2} U_{7}\right)-A_{1}\left(T_{3} U_{10}+T_{2} U_{7}\right)+\gamma_{p}\left(U_{3} U_{10}+U_{2} U_{7}\right)}{R_{6} U_{10}}-\frac{U_{7} \theta_{c}}{U_{10}}+A_{3}\right] \\
& \cdot\left[\frac{\left(\gamma_{c}+\gamma_{p}\right)\left(R_{5} U_{10}+R_{2} U_{9}\right)+\gamma_{p}\left(T_{5} U_{10}+T_{2} U_{9}\right)-A_{2}\left(U_{5} U_{10}+U_{2} U_{9}\right)}{R_{6} U_{10}}-\frac{U_{9}\left(\theta_{c}+\theta_{p}\right)}{U_{10}}+\varphi_{1}\right] \\
& \left.+\frac{U_{5} U_{10}+U_{2} U_{9}}{R_{6} U_{10}}\left[\frac{\left(\gamma_{c}+\gamma_{p}\right)\left(R_{3} U_{10}+R_{2} U_{7}\right)+\gamma_{p}\left(T_{3} U_{10}+T_{2} U_{7}\right)-A_{2}\left(U_{3} U_{10}+U_{2} U_{7}\right)}{R_{6} U_{10}}\right]-\frac{U_{7}\left(\theta_{c}+\theta_{p}\right)}{U_{10}}-\theta_{p}\right] \text {, } \\
& X_{1}=\frac{R_{4} U_{10}+R_{2} U_{8}}{2 R_{6} U_{10}}\left[\alpha_{r}+\frac{-\left(A_{1}+\gamma_{c}\right)\left(R_{1} U_{10}+R_{2} U_{6}\right)+\gamma_{c}\left(T_{1} U_{10}+T_{2} U_{6}\right)+\left(\gamma_{c}+\gamma_{p}\right)\left(U_{1} U_{10}+U_{2} U_{6}\right)}{R_{6} U_{10}}+\frac{U_{6}\left(A_{3}+\theta_{c}\right)}{U_{10}}\right] \\
& +\frac{1}{2}\left(\frac{R_{1} U_{10}+R_{2} U_{6}}{R_{6} U_{10}}-c_{s}\right) \\
& \cdot\left[\frac{-\left(A_{1}+\gamma_{c}\right)\left(R_{4} U_{10}+R_{2} U_{8}\right)+\gamma_{c}\left(T_{4} U_{10}+T_{2} U_{8}\right)+\left(\gamma_{c}+\gamma_{p}\right)\left(U_{4} U_{10}+U_{2} U_{8}\right)}{R_{6} U_{10}}+\frac{U_{8}\left(A_{3}+\theta_{c}\right)}{U_{10}}-\theta_{c}-\theta_{p}\right] \\
& +\frac{T_{4} U_{10}+T_{2} U_{8}}{R_{6} U_{10}}\left[\alpha_{m}+\frac{\gamma_{c}\left(R_{1} U_{10}+R_{2} U_{6}\right)-A_{1}\left(T_{1} U_{10}+T_{2} U_{6}\right)+\gamma_{p}\left(U_{1} U_{10}+U_{2} U_{6}\right)}{R_{6} U_{10}}-\frac{U_{6} \theta_{c}}{U_{10}}\right] \\
& +\left(\frac{T_{1} U_{10}+T_{2} U_{6}}{R_{6} U_{10}}-c_{s}\right)\left[\frac{\gamma_{c}\left(R_{4} U_{10}+R_{2} U_{8}\right)-A_{1}\left(T_{4} U_{10}+T_{2} U_{8}\right)+\gamma_{p}\left(U_{4} U_{10}+U_{2} U_{8}\right)}{R_{6} U_{10}}-\frac{U_{8} \theta_{c}}{U_{10}}-\theta_{p}\right] \\
& +\frac{U_{4} U_{10}+U_{2} U_{8}}{R_{6} U_{10}}\left[\alpha_{z}+\frac{\left(\gamma_{c}+\gamma_{p}\right)\left(R_{1} U_{10}+R_{2} U_{6}\right)+\gamma_{p}\left(T_{1} U_{10}+T_{2} U_{6}\right)-A_{2}\left(U_{1} U_{10}+U_{2} U_{6}\right)}{R_{6} U_{10}}-\frac{U_{6}\left(\theta_{c}+\theta_{p}\right)}{U_{10}}\right] \\
& +\left(\frac{U_{1} U_{10}+U_{2} U_{6}}{R_{6} U_{10}}-c_{z}\right)\left[\frac{\left(\gamma_{c}+\gamma_{p}\right)\left(R_{4} U_{10}+R_{2} U_{8}\right)+\gamma_{p}\left(T_{4} U_{10}+T_{2} U_{8}\right)-A_{2}\left(U_{4} U_{10}+U_{2} U_{8}\right) U_{8}\left(\theta_{c}+\theta_{p}\right)}{R_{6} U_{10}}+A_{4}\right],
\end{aligned}
$$




$$
\begin{aligned}
& X_{2}=V_{3} \text {, } \\
& X_{3}=\frac{R_{4} U_{10}+R_{2} U_{8}}{R_{6} U_{10}}\left[\frac{-\left(A_{1}+\gamma_{c}\right)\left(R_{4} U_{10}+R_{2} U_{8}\right)+\gamma_{c}\left(T_{4} U_{10}+T_{2} U_{8}\right)+\left(\gamma_{c}+\gamma_{p}\right)\left(U_{4} U_{10}+U_{2} U_{8}\right)}{R_{6} U_{10}}+\frac{U_{8}\left(A_{3}+\theta_{c}\right)}{U_{10}}-\theta_{c}-\theta_{p}\right] \\
& +\frac{2\left(T_{4} U_{10}+T_{2} U_{8}\right)}{R_{6} U_{10}}\left[\frac{\gamma_{c}\left(R_{4} U_{10}+R_{2} U_{8}\right)-A_{1}\left(T_{4} U_{10}+T_{2} U_{8}\right)+\gamma_{p}\left(U_{4} U_{10}+U_{2} U_{8}\right)}{R_{6} U_{10}}-\frac{U_{8} \theta_{c}}{U_{10}}-\theta_{p}\right] \\
& +\frac{2\left(U_{4} U_{10}+U_{2} U_{8}\right)}{R_{6} U_{10}}\left[\frac{\left(\gamma_{c}+\gamma_{p}\right)\left(R_{4} U_{10}+R_{2} U_{8}\right)+\gamma_{p}\left(T_{4} U_{10}+T_{2} U_{8}\right)-A_{2}\left(U_{4} U_{10}+U_{2} U_{8}\right)}{R_{6} U_{10}}-\frac{U_{8}\left(\theta_{c}+\theta_{p}\right)}{U_{10}}+A_{4}\right]-\eta_{1} \text {, } \\
& Y_{2}=\frac{R_{5} U_{10}+R_{2} U_{9}}{2 R_{6} U_{10}}\left[\frac{-\left(A_{1}+\gamma_{c}\right)\left(R_{3} U_{10}+R_{2} U_{7}\right)+\gamma_{c}\left(T_{3} U_{10}+T_{2} U_{7}\right)+\left(\gamma_{c}+\gamma_{p}\right)\left(U_{3} U_{10}+U_{2} U_{7}\right)}{R_{6} U_{10}}+\frac{U_{7}\left(A_{3}+\theta_{c}\right)}{U_{10}}-\theta_{c}\right] \\
& +\frac{R_{3} U_{10}+R_{2} U_{7}}{2 R_{6} U_{10}} \\
& \cdot\left[\frac{-\left(A_{1}+\gamma_{c}\right)\left(R_{5} U_{10}+R_{2} U_{9}\right)+\gamma_{c}\left(T_{5} U_{10}+T_{2} U_{9}\right)+\left(\gamma_{c}+\gamma_{p}\right)\left(U_{5} U_{10}+U_{2} U_{9}\right)}{R_{6} U_{10}}+\frac{U_{9}\left(A_{3}+\theta_{c}\right)}{U_{10}}-\varphi_{2}\right] \\
& +\frac{T_{5} U_{10}+T_{2} U_{9}}{R_{6} U_{10}}\left[\frac{\gamma_{c}\left(R_{3} U_{10}+R_{2} U_{7}\right)-A_{1}\left(T_{3} U_{10}+T_{2} U_{7}\right)+\gamma_{p}\left(U_{3} U_{10}+U_{2} U_{7}\right)}{R_{6} U_{10}}-\frac{U_{7} \theta_{c}}{U_{10}}+A_{3}\right] \\
& +\frac{T_{3} U_{10}+T_{2} U_{7}}{R_{6} U_{10}}\left[\frac{\gamma_{c}\left(R_{5} U_{10}+R_{2} U_{9}\right)-A_{1}\left(T_{5} U_{10}+T_{2} U_{9}\right)+\gamma_{p}\left(U_{5} U_{10}+U_{2} U_{9}\right)}{R_{6} U_{10}}-\frac{U_{9} \theta_{c}}{U_{10}}-\varphi_{2}\right] \\
& +\frac{U_{5} U_{10}+U_{2} U_{9}}{R_{6} U_{10}}\left[\frac{\left(\gamma_{c}+\gamma_{p}\right)\left(R_{3} U_{10}+R_{2} U_{7}\right)+\gamma_{p}\left(T_{3} U_{10}+T_{2} U_{7}\right)-A_{2}\left(U_{3} U_{10}+U_{2} U_{7}\right)}{R_{6} U_{10}}-\frac{U_{7}\left(\theta_{c}+\theta_{p}\right)}{U_{10}}-\theta_{p}\right] \\
& +\frac{U_{3} U_{10}+U_{2} U_{7}}{R_{6} U_{10}}\left[\frac{\left(\gamma_{c}+\gamma_{p}\right)\left(R_{5} U_{10}+R_{2} U_{9}\right)+\gamma_{p}\left(T_{5} U_{10}+T_{2} U_{9}\right)-A_{2}\left(U_{5} U_{10}+U_{2} U_{9}\right)}{R_{6} U_{10}}-\frac{U_{9}\left(\theta_{c}+\theta_{p}\right)}{U_{10}}+\varphi_{1}\right], \\
& Y_{3}=X_{4} \text {, } \\
& Y_{4}=\frac{R_{5} U_{10}+R_{2} U_{9}}{R_{6} U_{10}}\left[\frac{-\left(A_{1}+\gamma_{c}\right)\left(R_{5} U_{10}+R_{2} U_{9}\right)+\gamma_{c}\left(T_{5} U_{10}+T_{2} U_{9}\right)+\left(\gamma_{c}+\gamma_{p}\right)\left(U_{5} U_{10}+U_{2} U_{9}\right)}{R_{6} U_{10}}+\frac{U_{9}\left(A_{3}+\theta_{c}\right)}{U_{10}}-\varphi_{2}\right] \\
& +\frac{2\left(T_{5} U_{10}+T_{2} U_{9}\right)}{R_{6} U_{10}}\left[\frac{\gamma_{c}\left(R_{5} U_{10}+R_{2} U_{9}\right)-A_{1}\left(T_{5} U_{10}+T_{2} U_{9}\right)+\gamma_{p}\left(U_{5} U_{10}+U_{2} U_{9}\right)}{R_{6} U_{10}}-\frac{U_{9} \theta_{c}}{U_{10}}-\varphi_{2}\right] \\
& +\frac{2\left(U_{5} U_{10}+U_{2} U_{9}\right)}{R_{6} U_{10}}\left[\frac{\left(\gamma_{c}+\gamma_{p}\right)\left(R_{5} U_{10}+R_{2} U_{9}\right)+\gamma_{p}\left(T_{5} U_{10}+T_{2} U_{9}\right)-A_{2}\left(U_{5} U_{10}+U_{2} U_{9}\right)}{R_{6} U_{10}}-\frac{U_{9}\left(\theta_{c}+\theta_{p}\right)}{U_{10}}+\varphi_{1}\right]-C .
\end{aligned}
$$

Proof of Proposition 11. Take the second-order partial derivatives of $\Pi_{r}$ with respect to $s,\left(\partial^{2} \Pi_{r} / \partial s^{2}\right)=2 \Theta_{1} \Theta_{2}-(1$ $\left.-\varphi_{0} \varphi\right) \eta_{2}$. To ensure that $\Pi_{r}$ is concave in $s,\left(\partial^{2} \Pi_{r} / \partial s^{2}\right)$ should be negative. Therefore, the optimal $s$ can be obtained provided that $2 \Theta_{1} \Theta_{2}<\left(1-\varphi_{0} \varphi\right) \eta_{2}, s^{*}=\left(\left[\alpha_{r}+\left(\gamma_{c} B_{2}+\left(\gamma_{c}\right.\right.\right.\right.$ $\left.\left.\left.+\gamma_{p}\right) B_{3}-\left(A_{1}+\gamma_{c}\right) B_{4} / A_{6}\right)\right] \Theta_{1}+\left(B_{4}-B_{1} / A_{6}\right) \Theta_{2} /\left(1-\varphi_{0}\right)$ $\left.\eta_{2}-2 \Theta_{1} \Theta_{2}\right)+\left(\left[\gamma_{c} G_{2}+\left(\gamma_{c}+\gamma_{p}\right) G_{3}-\left(A_{1}+\gamma_{c}\right) G_{4}-\varphi_{2} A_{6}\right]\right.$ $\left.\Theta_{1}+\left(G_{4}-G_{1}\right) \Theta_{2} / A_{6}\left[\left(1-\varphi_{0}\right) \eta_{2}-2 \Theta_{1} \Theta_{2}\right]\right) \mu$, where 


$$
\begin{aligned}
& \Theta_{1}=-\frac{1}{2}\left\{\frac{1}{2} \beta_{s} A_{5}-2\left[\gamma_{c} A_{2}+\gamma_{p}\left(\gamma_{c}+\gamma_{p}\right)\right]\left[\frac{\gamma_{c} \beta_{s}}{2\left(A_{1}+\gamma_{c}\right)}+\beta_{s}\right]-2\left[A_{1}\left(\gamma_{c}+\gamma_{p}\right)+\gamma_{c} \gamma_{p}\right]\left[\frac{\left(\gamma_{c}+\gamma_{p}\right) \beta_{s}}{2\left(A_{1}+\gamma_{c}\right)}+\beta_{z}\right]\right\} \\
& +\frac{\gamma_{c}}{2\left(A_{1}+\gamma_{c}\right)}\left\{-\beta_{s}\left[\gamma_{c} A_{2}+\gamma_{p}\left(\gamma_{c}+\gamma_{p}\right)\right]-2\left[\left(\gamma_{c}+\gamma_{p}\right)^{2}-\left(A_{1}+\gamma_{c}\right) A_{2}\right]\left[\frac{\gamma_{c} \beta_{s}}{2\left(A_{1}+\gamma_{c}\right)}+\beta_{s}\right]\right. \\
& \left.-2\left[\gamma_{c}\left(\gamma_{c}+\gamma_{p}\right)+\gamma_{p}\left(A_{1}+\gamma_{c}\right)\right]\left[\frac{\left(\gamma_{c}+\gamma_{p}\right) \beta_{s}}{2\left(A_{1}+\gamma_{c}\right)}+\beta_{z}\right]\right\}+\frac{\gamma_{c}+\gamma_{p}}{2\left(A_{1}+\gamma_{c}\right)}\left\{-\beta_{s}\left[A_{1}\left(\gamma_{c}+\gamma_{p}\right)+\gamma_{c} \gamma_{p}\right]\right. \\
& \left.-2\left[\gamma_{c}\left(\gamma_{c}+\gamma_{p}\right)+\gamma_{p}\left(A_{1}+\gamma_{c}\right)\right]\left[\frac{\gamma_{c} \beta_{s}}{2\left(A_{1}+\gamma_{c}\right)}+\beta_{s}\right]+2\left[\gamma_{c}^{2}-\left(A_{1}+\gamma_{c}\right) A_{1}\right]\left[\frac{\left(\gamma_{c}+\gamma_{p}\right) \beta_{s}}{2\left(A_{1}+\gamma_{c}\right)}+\beta_{z}\right]\right\} \\
& +\frac{\beta_{s} A_{6}}{2\left(A_{1}+\gamma_{c}\right)}, \\
& \Theta_{2}=-\frac{A_{1}+\gamma_{c}}{A_{6}}\left(\frac { 1 } { 2 } \left\{\frac{1}{2} \beta_{s} A_{5}-2\left[\gamma_{c} A_{2}+\gamma_{p}\left(\gamma_{c}+\gamma_{p}\right)\right]\left[\frac{\gamma_{c} \beta_{s}}{2\left(A_{1}+\gamma_{c}\right)}+\beta_{s}\right]-2\left[A_{1}\left(\gamma_{c}+\gamma_{p}\right)+\gamma_{c} \gamma_{p}\right]\right.\right. \\
& \left.\cdot\left[\frac{\left(\gamma_{c}+\gamma_{p}\right) \beta_{s}}{2\left(A_{1}+\gamma_{c}\right)}+\beta_{z}\right]\right\}+\frac{\gamma_{c}}{2\left(A_{1}+\gamma_{c}\right)}\left\{-\beta_{s}\left[\gamma_{c} A_{2}+\gamma_{p}\left(\gamma_{c}+\gamma_{p}\right)\right]-2\left[\left(\gamma_{c}+\gamma_{p}\right)^{2}-\left(A_{1}+\gamma_{c}\right) A_{2}\right]\left[\frac{\gamma_{c} \beta_{s}}{2\left(A_{1}+\gamma_{c}\right)}+\beta_{s}\right]\right. \\
& \left.-2\left[\gamma_{c}\left(\gamma_{c}+\gamma_{p}\right)+\gamma_{p}\left(A_{1}+\gamma_{c}\right)\right]\left[\frac{\left(\gamma_{c}+\gamma_{p}\right) \beta_{s}}{2\left(A_{1}+\gamma_{c}\right)}+\beta_{z}\right]\right\}+\frac{\gamma_{c}+\gamma_{p}}{2\left(A_{1}+\gamma_{c}\right)}\left\{-\beta_{s}\left[A_{1}\left(\gamma_{c}+\gamma_{p}\right)+\gamma_{c} \gamma_{p}\right]\right. \\
& \left.-2\left[\gamma_{c}\left(\gamma_{c}+\gamma_{p}\right)+\gamma_{p}\left(A_{1}+\gamma_{c}\right)\right]\left[\frac{\gamma_{c} \beta_{s}}{2\left(A_{1}+\gamma_{c}\right)}+\beta_{s}\right]+2\left[\gamma_{c}^{2}-\left(A_{1}+\gamma_{c}\right) A_{1}\right]\left[\frac{\left(\gamma_{c}+\gamma_{p}\right) \beta_{s}}{2\left(A_{1}+\gamma_{c}\right)}+\beta_{z}\right]\right\} \\
& \left.+\frac{\beta_{s} A_{6}}{2\left(A_{1}+\gamma_{c}\right)}\right)+\frac{\gamma_{c}}{A_{6}}\left\{-\beta_{s}\left[\gamma_{c} A_{2}+\gamma_{p}\left(\gamma_{c}+\gamma_{p}\right)\right]-2\left[\left(\gamma_{c}+\gamma_{p}\right)^{2}-\left(A_{1}+\gamma_{c}\right) A_{2}\right]\left[\frac{\gamma_{c} \beta_{s}}{2\left(A_{1}+\gamma_{c}\right)}+\beta_{s}\right]\right. \\
& \left.-2\left[\gamma_{c}\left(\gamma_{c}+\gamma_{p}\right)+\gamma_{p}\left(A_{1}+\gamma_{c}\right)\right]\left[\frac{\left(\gamma_{c}+\gamma_{p}\right) \beta_{s}}{2\left(A_{1}+\gamma_{c}\right)}+\beta_{z}\right]\right\}+\frac{\gamma_{c}+\gamma_{p}}{A_{6}}\left\{-\beta_{s}\left[A_{1}\left(\gamma_{c}+\gamma_{p}\right)+\gamma_{c} \gamma_{p}\right]\right. \\
& \left.-2\left[\gamma_{c}\left(\gamma_{c}+\gamma_{p}\right)+\gamma_{p}\left(A_{1}+\gamma_{c}\right)\right]\left[\frac{\gamma_{c} \beta_{s}}{2\left(A_{1}+\gamma_{c}\right)}+\beta_{s}\right]+2\left[\gamma_{c}^{2}-\left(A_{1}+\gamma_{c}\right) A_{1}\right]\left[\frac{\left(\gamma_{c}+\gamma_{p}\right) \beta_{s}}{2\left(A_{1}+\gamma_{c}\right)}+\beta_{z}\right]\right\}+\beta_{s} .
\end{aligned}
$$

Proof of Proposition 12. Take the second-order partial derivatives of $\Pi_{m}$ with respect to $\mu\left(\partial^{2} \Pi_{m} / \partial \mu^{2}\right)=$ $-C-\varphi_{0} \eta_{2} Z_{10}^{2}+2 Z_{2} \quad\left[-\left(A_{1}+\gamma_{c}\right) Z_{8}+\gamma_{c} Z_{4}+\left(\gamma_{c}+\gamma_{p}\right) Z_{6}+\right.$ $\left.\beta_{s} Z_{10}-\varphi_{2}\right]+2 Z_{4}\left(\gamma_{c} Z_{8}-A_{1} Z_{4}+\gamma_{p} Z_{6}+\beta_{s} Z_{10}-\varphi_{2}\right)+2 Z_{6}$ $\left[\left(\gamma_{c}+\gamma_{p}\right) Z_{8}+\gamma_{p} Z_{4}-A_{2} Z_{6}+\beta_{z} Z_{10}+\varphi_{1}\right]$ To ensure that
$\Pi_{m}$ is concave in $\mu,\left(\partial^{2} \Pi_{m} / \partial \mu^{2}\right)$ should be negative. Therefore, the optimal $s$ can be obtained provided that $C+\varphi_{0} \eta_{2} Z_{10}^{2}-2 Z_{2}\left[-\left(A_{1}+\gamma_{c}\right) Z_{8}+\gamma_{c} Z_{4}+\left(\gamma_{c}+\gamma_{p}\right) Z_{6}+\right.$ $\left.\beta_{s} Z_{10}-\varphi_{2}\right]-2 Z_{4} \quad\left(\gamma_{c} Z_{8}-A_{1} Z_{4}+\gamma_{p} Z_{6}+\beta_{s} Z_{10}-\varphi_{2}\right)-$ $2 Z_{6}\left[\left(\gamma_{c}+\gamma_{p}\right) Z_{8}+\gamma_{p} Z_{4}-A_{2} Z_{6}+\beta_{z} Z_{10}+\varphi_{1}\right]>0$, where 


$$
\begin{aligned}
& Z_{9}=\frac{\left[\alpha_{r}+\left(\gamma_{c} B_{2}+\left(\gamma_{c}+\gamma_{p}\right) B_{3}-\left(A_{1}+\gamma_{c}\right) B_{4} / A_{6}\right)\right] \Theta_{1}+\left(B_{4}-B_{1} / A_{6}\right) \Theta_{2}}{\left(1-\varphi_{0}\right) \eta_{2}-2 \Theta_{1} \Theta_{2}} \\
& Z_{10}=\frac{\left[\gamma_{c} G_{2}+\left(\gamma_{c}+\gamma_{p}\right) G_{3}-\left(A_{1}+\gamma_{c}\right) G_{4}-\varphi_{2} A_{6}\right] \Theta_{1}+\left(G_{4}-G_{1}\right) \Theta_{2}}{A_{6}\left[\left(1-\varphi_{0}\right) \eta_{2}-2 \Theta_{1} \Theta_{2}\right]} \\
& Z_{1}=\frac{B_{1}+Z_{9}\left\{(1 / 2) \beta_{s} A_{5}-2\left[\gamma_{c} A_{2}+\gamma_{p}\left(\gamma_{c}+\gamma_{p}\right)\right]\left[\left(\gamma_{c} \beta_{s} / 2\left(A_{1}+\gamma_{c}\right)\right)+\beta_{s}\right]-2\left[A_{1}\left(\gamma_{c}+\gamma_{p}\right)+\gamma_{c} \gamma_{p}\right]\left[\left(\left(\gamma_{c}+\gamma_{p}\right) \beta_{s} / 2\left(A_{1}+\gamma_{c}\right)\right)+\beta_{z}\right]\right\}}{A_{6}}, \\
& Z_{2}=\frac{G_{1}+Z_{10}\left\{1 / 2 \beta_{s} A_{5}-2\left[\gamma_{c} A_{2}+\gamma_{p}\left(\gamma_{c}+\gamma_{p}\right)\right]\left[\left(\gamma_{c} \beta_{s} / 2\left(A_{1}+\gamma_{c}\right)\right)+\beta_{s}\right]-2\left[A_{1}\left(\gamma_{c}+\gamma_{p}\right)+\gamma_{c} \gamma_{p}\right]\left[\left(\left(\gamma_{c}+\gamma_{p}\right) \beta_{s} / 2\left(A_{1}+\gamma_{c}\right)\right)+\beta_{z}\right]\right\}}{A_{6}}, \\
& Z_{3}=\frac{B_{2}-Z_{9}\left\{\beta_{s}\left[\gamma_{c} A_{2}+\gamma_{p}\left(\gamma_{c}+\gamma_{p}\right)\right]-2\left[\left(\gamma_{c}+\gamma_{p}\right)^{2}-\left(A_{1}+\gamma_{c}\right) A_{2}\right]\left[\left(\gamma_{c} \beta_{s} / 2\left(A_{1}+\gamma_{c}\right)\right)+\beta_{s}\right]\right\}}{A_{6}} \\
& -\frac{2\left[\gamma_{c}\left(\gamma_{c}+\gamma_{p}\right)+\gamma_{p}\left(A_{1}+\gamma_{c}\right)\right]\left[\left(\left(\gamma_{c}+\gamma_{p}\right) \beta_{s} / 2\left(A_{1}+\gamma_{c}\right)\right)+\beta_{z}\right]}{A_{6}} \\
& Z_{4}=\frac{G_{2}-Z_{10}\left\{\beta_{s}\left[\gamma_{c} A_{2}+\gamma_{p}\left(\gamma_{c}+\gamma_{p}\right)\right]+2\left[\left(\gamma_{c}+\gamma_{p}\right)^{2}-\left(A_{1}+\gamma_{c}\right) A_{2}\right]\left[\left(\gamma_{c} \beta_{s} / 2\left(A_{1}+\gamma_{c}\right)\right)+\beta_{s}\right]\right\}}{A_{6}} \\
& -\frac{2\left[\gamma_{c}\left(\gamma_{c}+\gamma_{p}\right)+\gamma_{p}\left(A_{1}+\gamma_{c}\right)\right]\left[\left(\left(\gamma_{c}+\gamma_{p}\right) \beta_{s} / 2\left(A_{1}+\gamma_{c}\right)\right)+\beta_{z}\right]}{A_{6}} \\
& Z_{5}=\frac{B_{3}-Z_{9}\left\{\beta_{s}\left[A_{1}\left(\gamma_{c}+\gamma_{p}\right)+\gamma_{c} \gamma_{p}\right]+2\left[\gamma_{c}\left(\gamma_{c}+\gamma_{p}\right)+\gamma_{p}\left(A_{1}+\gamma_{c}\right)\right]\left[\left(\gamma_{c} \beta_{s} / 2\left(A_{1}+\gamma_{c}\right)\right)+\beta_{s}\right]\right\}}{A_{6}} \\
& +\frac{2\left[\gamma_{c}^{2}-\left(A_{1}+\gamma_{c}\right) A_{1}\right]\left[\left(\left(\gamma_{c}+\gamma_{p}\right) \beta_{s} / 2\left(A_{1}+\gamma_{c}\right)\right)+\beta_{z}\right]}{A_{6}}, \\
& Z_{6}=\frac{G_{3}-Z_{10}\left\{\beta_{s}\left[A_{1}\left(\gamma_{c}+\gamma_{p}\right)+\gamma_{c} \gamma_{p}\right]+2\left[\gamma_{c}\left(\gamma_{c}+\gamma_{p}\right)+\gamma_{p}\left(A_{1}+\gamma_{c}\right)\right]\left[\left(\gamma_{c} \beta_{s} / 2\left(A_{1}+\gamma_{c}\right)\right)+\beta_{s}\right]\right\}}{A_{6}} \\
& +\frac{2\left[\gamma_{c}^{2}-\left(A_{1}+\gamma_{c}\right) A_{1}\right]\left[\left(\left(\gamma_{c}+\gamma_{p}\right) \beta_{s} / 2\left(A_{1}+\gamma_{c}\right)\right)+\beta_{z}\right]}{A_{6}}, \\
& Z_{7}=\frac{Z_{1}}{2}+\frac{\alpha_{r}+\gamma_{c} Z_{3}+\left(\gamma_{c}+\gamma_{p}\right) Z_{5}+\beta_{s} Z_{9}}{2\left(A_{1}+\gamma_{c}\right)} \\
& Z_{8}=\frac{Z_{2}}{2}+\frac{\gamma_{c} Z_{4}+\left(\gamma_{c}+\gamma_{p}\right) Z_{6}+\beta_{s} Z_{10}-\varphi_{2}}{2\left(A_{1}+\gamma_{c}\right)}, \\
& Z_{11}=C+\varphi_{0} \eta_{2} Z_{10}^{2}-2 Z_{2}\left[-\left(A_{1}+\gamma_{c}\right) Z_{8}+\gamma_{c} Z_{4}+\left(\gamma_{c}+\gamma_{p}\right) Z_{6}+\beta_{s} Z_{10}-\varphi_{2}\right] \\
& -2 Z_{4}\left(\gamma_{c} Z_{8}-A_{1} Z_{4}+\gamma_{p} Z_{6}+\beta_{s} Z_{10}-\varphi_{2}\right)-2 Z_{6}\left[\left(\gamma_{c}+\gamma_{p}\right) Z_{8}+\gamma_{p} Z_{4}-A_{2} Z_{6}+\beta_{z} Z_{10}+\varphi_{1}\right] .
\end{aligned}
$$




\section{Data Availability}

The data used to support the findings of this study are included within the article.

\section{Conflicts of Interest}

The authors declare that they have no conflicts of interest.

\section{Acknowledgments}

The work described in this article was supported by National Natural Science Foundation of China (NSFC) under project nos.72071084 and 71671071.

\section{References}

[1] J. Xie, W. Zhang, L. Liang, Y. Xia, J. Yin, and G. Yang, "The revenue and cost sharing contract of pricing and servicing policies in a dual-channel closed-loop supply chain," Journal of Cleaner Production, vol. 191, pp. 361-383, 2018.

[2] B. C. Giri, A. Chakraborty, and T. Maiti, "Pricing and return product collection decisions in a closed-loop supply chain with dual-channel in both forward and reverse logistics," Journal of Manufacturing Systems, vol. 42, pp. 104-123, 2017.

[3] L. Kong, Z. Liu, Y. Pan, J. Xie, and G. Yang, "Pricing and service decision of dual-channel operations in an $\mathrm{O} 2 \mathrm{O}$ closedloop supply chain," Industrial Management \& Data Systems, vol. 117, no. 8, pp. 1567-1588, 2017.

[4] W. Zhao, C. Ye, and X. Ding, "Preferred service investment in the dual-channel supply chain: leader-follower relationships and product characteristics," Mathematical Problems in Engineering, vol. 2020, Article ID 1656094, 22 pages, 2020.

[5] F. L. S. Chua, N. N. Vasnani, L. B. M. Pacio, and L. A. Ocampo, "A Stackelberg game in multi-period planning of make-to-order production system across the supply chain," Journal of Manufacturing Systems, vol. 46, pp. 231-246, 2018.

[6] M. Childs and B. Jin, Nike: An Innovation Journey, Palgrave Macmillan US, London, UK, 2018.

[7] D. Wu, J. Chen, P. Li, and R. Zhang, "Contract coordination of dual channel reverse supply chain considering service level," Journal of Cleaner Production, vol. 260, p. 121071, 2020.

[8] X. Ma, S. Wang, S. M. N. Islam, and X. Liu, "Coordinating a three-echelon fresh agricultural products supply chain considering freshness-keeping effort with asymmetric information," Applied Mathematical Modelling, vol. 67, pp. 337-356, 2019.

[9] M. Dell and C. Fredman, Direct from Dell: Strategies that Revolutionized an Industry, SAGE Publications, Thousand Oaks, CA, USA, 1st edition, 2002.

[10] X. Sun, Q. Zhang, and Y. Zhou, "Service and price decisions of a supply chain with optional after-sale service," Mathematical Problems in Engineering, vol. 2016, Article ID 1505817, 11 pages, 2016.

[11] Y. Yu and T. Xiao, "Pricing and cold-chain service level decisions in a fresh agri-products supply chain with logistics outsourcing," Computers \& Industrial Engineering, vol. 111, pp. 56-66, 2017.

[12] J.-C. Lu, Y.-C. Tsao, and C. Charoensiriwath, "Competition under manufacturer service and retail price," Economic Modelling, vol. 28, no. 3, pp. 1256-1264, 2011.

[13] C.-H. Wu, "Price and service competition between new and remanufactured products in a two-echelon supply chain,"
International Journal of Production Economics, vol. 140, no. 1, pp. 496-507, 2012.

[14] B. Wang and J. Wang, "Price and service competition between new and remanufactured products," Mathematical Problems in Engineering, vol. 140, Article ID 325185, 2015.

[15] B. Dan, Z. J. Qu, C. Liu, X. M. Zhang, and H. Y. Zhang, "Price and service competition in the supply chain with both pure play internet and strong bricks-and-mortar retailers," Journal of Applied Research and Technology, vol. 12, no. 2, pp. 212222, 2014

[16] X. Han, X. Sun, and Y. Zhou, "The equilibrium decisions in a two-echelon supply chain under price and service competition," Sustainability, vol. 6, no. 7, pp. 4339-4354, 2014.

[17] Q. Xu, B. Xu, Q. Bo, and Y. He, "Coordination through cooperative advertising in a two-period supply chain with retail competition," Kybernetes, vol. 48, no. 6, pp. 1175-1194, 2019.

[18] J. Ma, Q. Li, and B. Bao, "Study on complex advertising and price competition dual-channel supply chain models considering the overconfidence manufacturer," Mathematical Problems in Engineering, vol. 2016, Article ID 2027146, 18 pages, 2016.

[19] B. Li, M. Zhu, Y. Jiang, and Z. Li, "Pricing policies of a competitive dual-channel green supply chain," Journal of Cleaner Production, vol. 112, pp. 2029-2042, 2016.

[20] C. Tang, H. Yang, E. Cao, and K. K. Lai, "Channel competition and coordination of a dual-channel supply chain with demand and cost disruptions," Applied Economics, vol. 50, no. 46, pp. 4999-5016, 2018.

[21] B. Dan, S. Zhang, and M. Zhou, "Strategies for warranty service in a dual-channel supply chain with value-added service competition," International Journal of Production Research, vol. 56, no. 17, pp. 5677-5699, 2018.

[22] A. Roy, S. S. Sana, and K. Chaudhuri, "Joint decision on EOQ and pricing strategy of a dual channel of mixed retail and e-tail comprising of single manufacturer and retailer under stochastic demand," Computers \& Industrial Engineering, vol. 102, pp. 423-434, 2016.

[23] L. Ren, Y. He, and H. Song, "Price and service competition of dual-channel supply chain with consumer returns," Discrete Dynamics in Nature and Society, vol. 2014, Article ID 565603, 10 pages, 2014.

[24] S. P. Sarmah, S. Sinha, and L. Kumar, "Price and warranty competition in a duopoly distribution channel: dynamic stability analysis for boundedly rational agents," IMA Journal of Management Mathematics, vol. 26, no. 3, pp. 299-324, 2015.

[25] G. Li, F. Huang, T. C. E. Cheng, and P. Ji, "Competition between manufacturer's online customization channel and conventional retailer," IEEE Transactions on Engineering Management, vol. 62, no. 2, pp. 150-157, 2015.

[26] T. Xiao, T.-M. Choi, and T. C. E. Cheng, "Product variety and channel structure strategy for a retailer-Stackelberg supply chain," European Journal of Operational Research, vol. 233, no. 1, pp. 114-124, 2014.

[27] R. Batarfi, M. Y. Jaber, and S. Zanoni, "Dual-channel supply chain: a strategy to maximize profit," Applied Mathematical Modelling, vol. 40, no. 21-22, pp. 9454-9473, 2016.

[28] R. Batarfi, M. Y. Jaber, and S. M. Aljazzar, "A profit maximization for a reverse logistics dual-channel supply chain with a return policy," Computers \& Industrial Engineering, vol. 106, pp. 58-82, 2017.

[29] Y. Li, "The study on pricing decision and revenue allocation of dual-channel supply chain under customization in ecology industry," Ekoloji, vol. 28, pp. 837-844, 2019. 
[30] L. Wang, Q. Song, and Z. Zhao, "The optimal pricing of dualchannel supply chain with the third party product recovery and sales effort," Complexity, vol. 2020, Article ID 4951341, 18 pages, 2020.

[31] J. Wei, J. Zhao, and X. Hou, "Bilateral information sharing in two supply chains with complementary products," Applied Mathematical Modelling, vol. 72, pp. 28-49, 2019.

[32] A. A. Tsay and N. Agrawal, "Channel dynamics under price and service competition," Manufacturing \& Service Operations Management, vol. 2, no. 4, pp. 372-391, 2000.

[33] Z.-Z. Zhang, Z.-J. Wang, and L.-W. Liu, "Retail services and pricing decisions in a closed-loop supply chain with remanufacturing," Sustainability, vol. 7, no. 3, pp. 2373-2396, 2015.

[34] D. Wu, "Joint pricing-servicing decision and channel strategies in the supply chain," Central European Journal of Operations Research, vol. 19, no. 1, pp. 99-137, 2011.

[35] J. Xie and A. Neyret, "Co-op advertising and pricing models in manufacturer-retailer supply chains," Computers \& Industrial Engineering, vol. 56, no. 4, pp. 1375-1385, 2009. 